Aus der Medizinischen Klinik

Abteilung Innere Medizin V (Pneumologie, Allergologie)

Universitätskliniken des Saarlandes

Homburg/Saar

Direktor: Prof. Dr. med. Dr. rer. nat. Robert Bals

\title{
Nicht-invasive Messung hämodynamischer Parameter bei Patienten mit pulmonalarterieller Hypertonie unter Belastung
}

\author{
Dissertation zur Erlangung des Grades eines \\ Doktors der Medizin \\ der Medizinischen Fakultät der Universität des Saarlandes
}

2013

vorgelegt von Kerstin Hadasch

geboren am 27.11.1981 in Finsterwalde 


\section{Inhaltsverzeichnis}

INHALTSVERZEICHNIS ...................................................................... I

ABKÜRZUNGSVERZEICHNIS.......................................................... IV

1 ZUSAMMENFASSUNG................................................................... 1

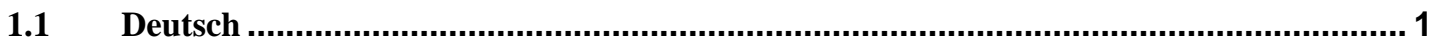

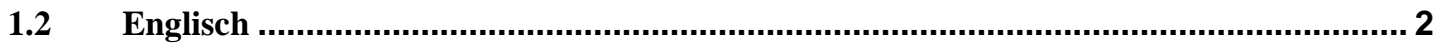

2 EINLEITUNG................................................................................... 4

$2.1 \quad$ Historisches zur pulmonalen Hypertonie ............................................................ 5

2.2 Klassifikation der pulmonalen Hypertonie …............................................................. 5

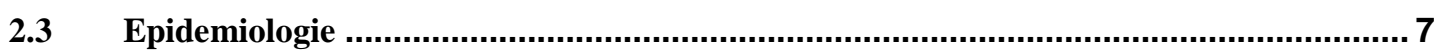

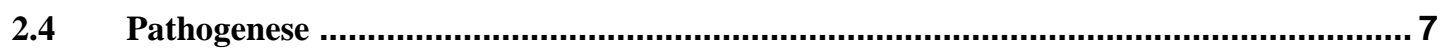

2.5 Beurteilung des Schweregrades der Erkrankung ........................................................

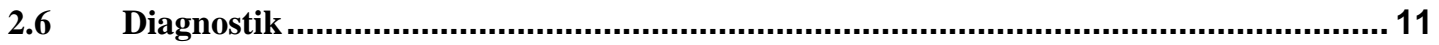

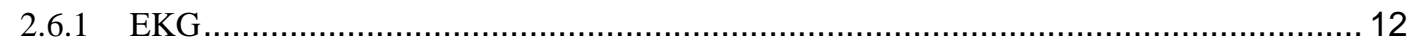

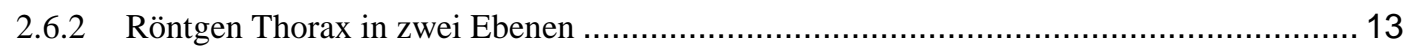

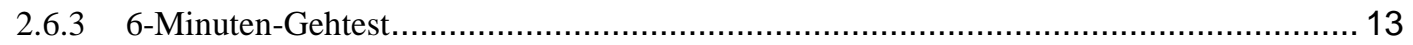

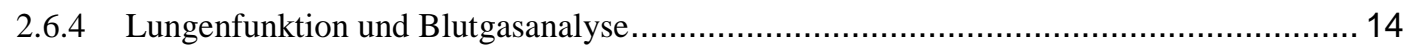

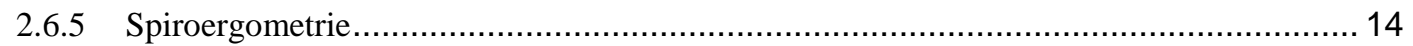

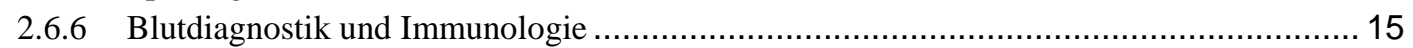

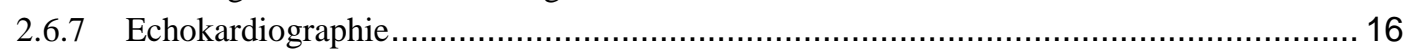

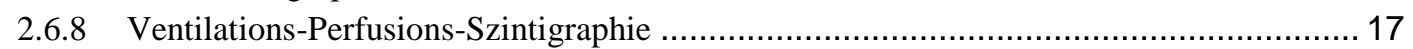

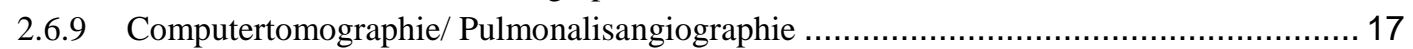

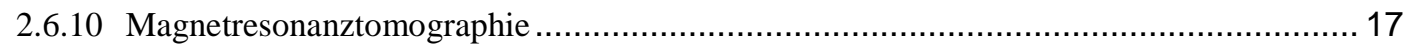

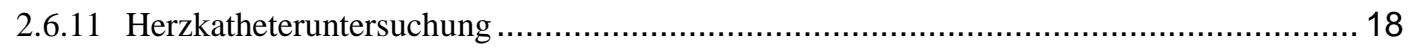

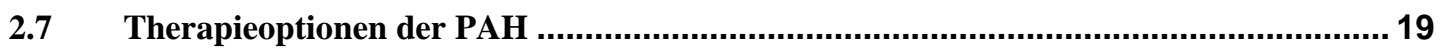

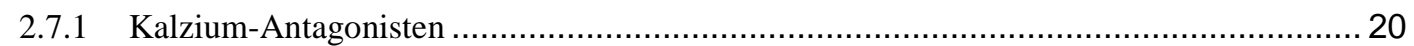

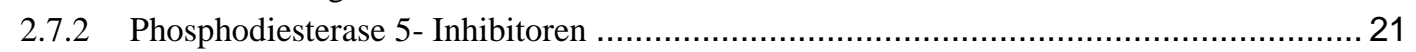

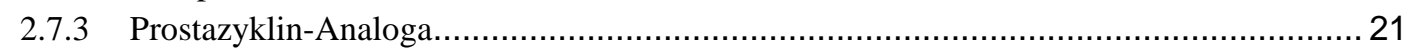

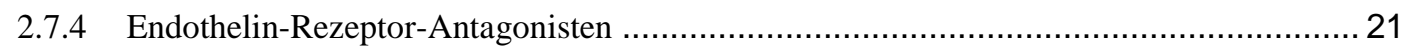

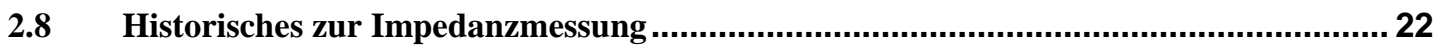

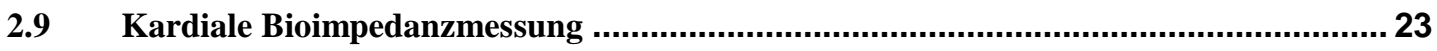

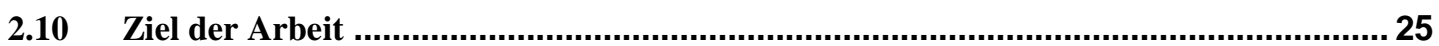




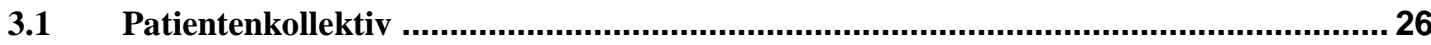

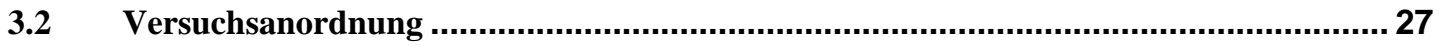

3.3 Parameter der kardialen Bioimpedanz ............................................................. 28

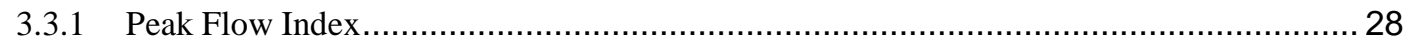

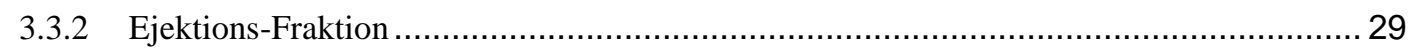

3.3.3 Enddiastolisches Volumen / Enddiastolischer Volumenindex ...................................... 30

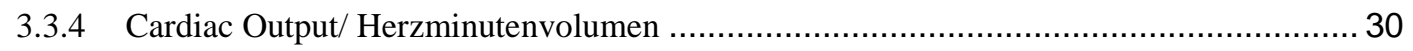

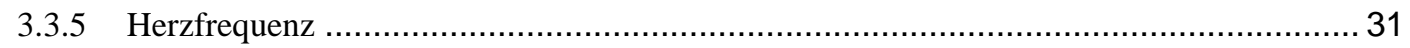

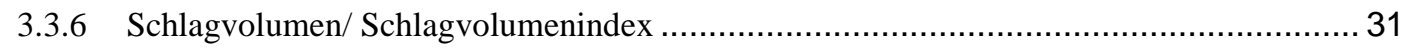

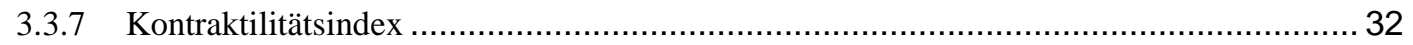

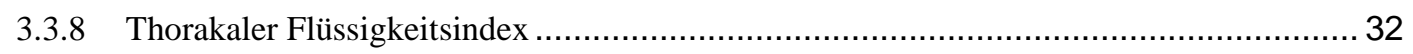

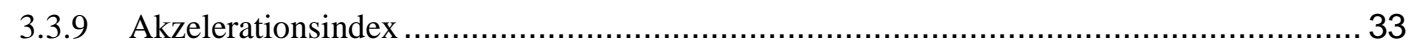

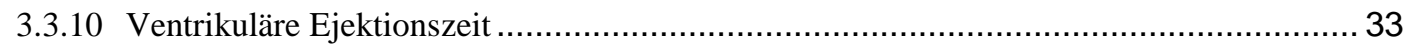

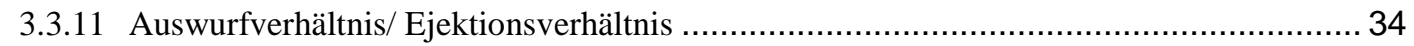

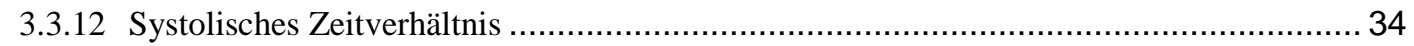

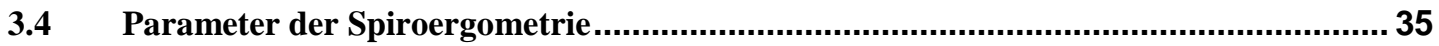

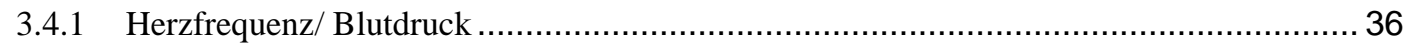

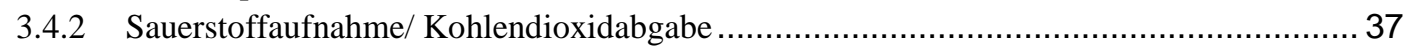

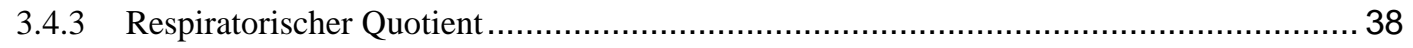

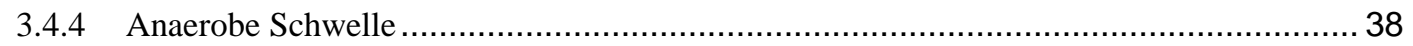

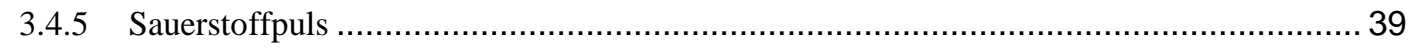

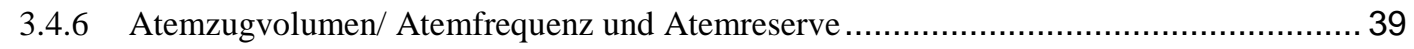

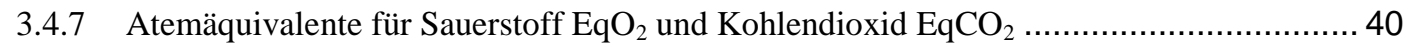

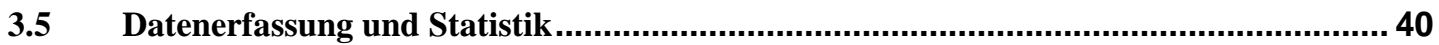

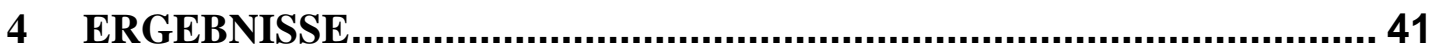

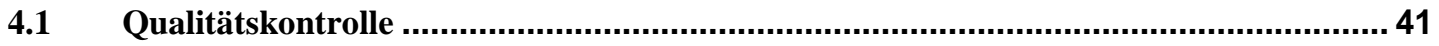

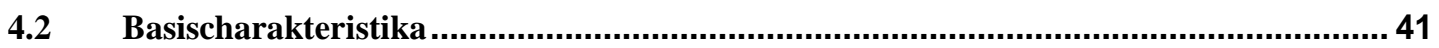

4.3 Medikation zum Zeitpunkt der Untersuchung ........................................................ 43

4.4 Vergleich der Sauerstoffaufnahme und Leistung (spiroergometrische Parameter) ...... 43

4.4.1 Vergleich der Leistungsfähigkeit an den jeweiligen Belastungszeitpunkten .................. 45

4.4.2 Sauerstoffaufnahme an den jeweiligen Belastungszeitpunkten ................................ 46

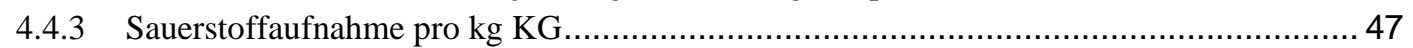

4.4.4 $\dot{\mathrm{VE}} / \dot{\mathrm{VCO}}_{2}$ an den jeweiligen Belastungszeitpunkten ........................................ 48

4.5 Darstellung der mittels kardialer Bioimpedanz ermittelten Parameter ....................... 49

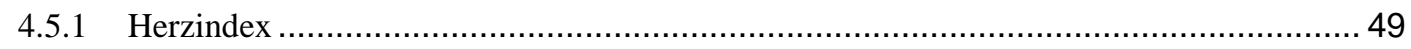

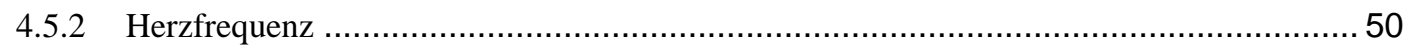

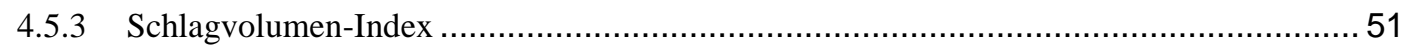

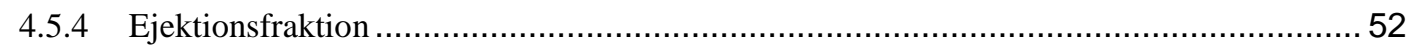

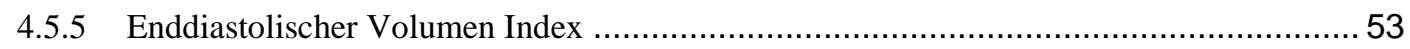

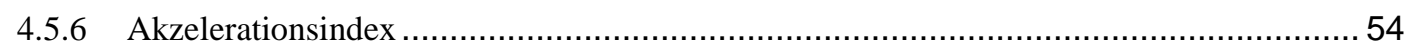




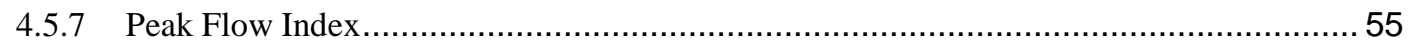

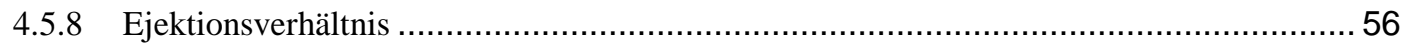

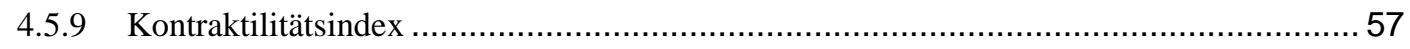

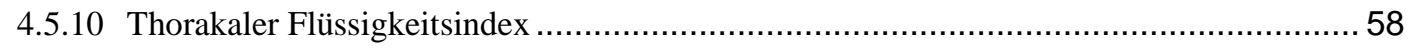

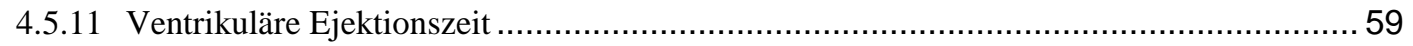

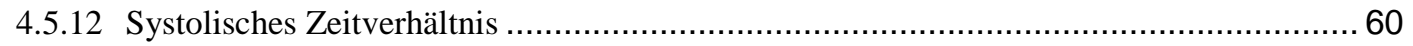

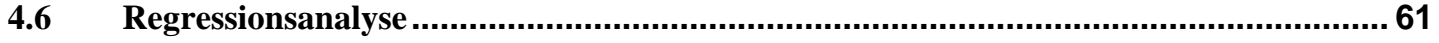

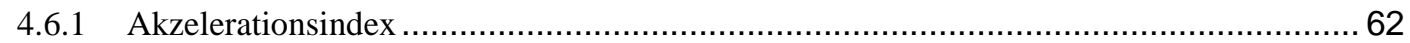

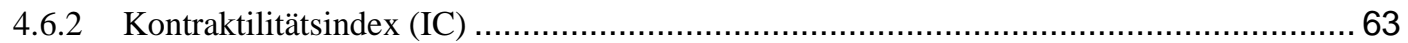

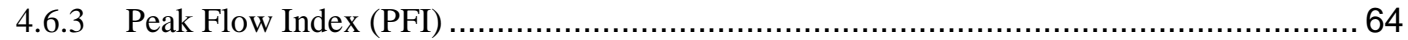

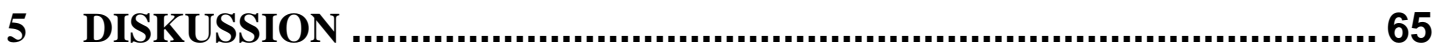

$5.1 \quad$ Stellenwert der thorakalen Impedanzkardiographie............................................6 65

5.2 Interpretation der hämodynamischen Veränderungen ...........................................66

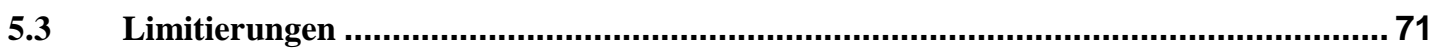

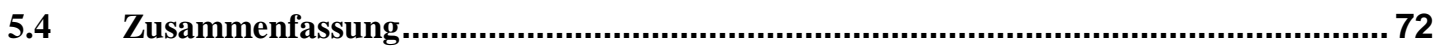

ABBILDUNGSVERZEICHNIS ............................................................ VII

TABELLENVERZEICHNIS................................................................ VIII

FORMELVERZEICHNIS .....................................................................IX

LITERATURVERZEICHNIS................................................................

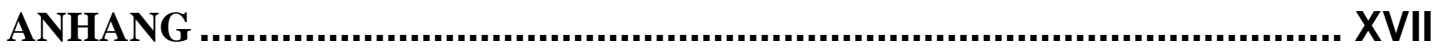

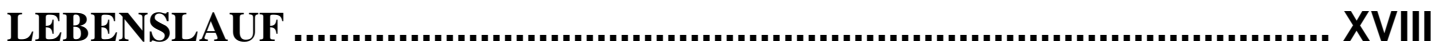

PUBLIKATIONEN

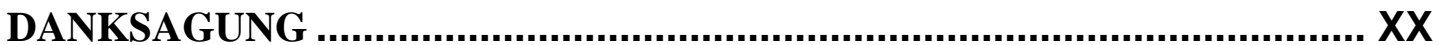




\section{Abkürzungsverzeichnis}

\begin{tabular}{|c|c|}
\hline 5-HTT & $\begin{array}{l}\text { 5-Hydroxytryptamintransporter } \\
\text { (Serotonintransporter) }\end{array}$ \\
\hline 6-MWT & 6-Minute-Walk-Test \\
\hline ACI & Akzelerationsindex - Beschleunigungsindex \\
\hline $\mathrm{AF}$ & Atemfrequenz \\
\hline ALK-1 & Actin-receptor-like Kinase-1 \\
\hline ANA & Antinukleäre Antikörper \\
\hline APAH & Associated Pulmonary Arterial Hypertension \\
\hline AT & Anaerobic Threshold \\
\hline $\mathrm{BL}$ & Baseline \\
\hline BMPR2 & Bone Morphogenetic Protein Receptor 2 \\
\hline $\mathrm{BNP}$ & Brain Natriuretic Peptide \\
\hline BR & Breathing Reserve - Atemreserve \\
\hline $\mathrm{BSA}=\mathrm{KOF}$ & Body Surface Area - Körperoberfläche \\
\hline cAMP & Zyklisches Adenosinmonophosphat \\
\hline cGMP & Zyklisches Guanosinmonophosphat \\
\hline CHD & $\begin{array}{l}\text { angeborene Herzfehler / Congenital Heart } \\
\text { Disease }\end{array}$ \\
\hline $\mathrm{CI}$ & Cardiac Index \\
\hline $\mathrm{CO}$ & Cardiac Output \\
\hline $\mathrm{CO}_{2}$ & Carbon Dioxid \\
\hline COPD & Chronic Obstructive Pulmonary Disease \\
\hline CTD & $\begin{array}{l}\text { systemische Bindegewebserkrankung } \\
\text { / Connective Tissue Disease }\end{array}$ \\
\hline CTEPH & $\begin{array}{l}\text { chronisch-thromboembolische pulmonale } \\
\text { Hypertonie }\end{array}$ \\
\hline $\mathrm{DL}_{\mathrm{CO}}$ & $\begin{array}{l}\text { Diffusion Capacity of the lung for Carbon } \\
\text { monoxide }\end{array}$ \\
\hline EDV & Enddiastolisches Volumen \\
\hline EDI & Enddiastolischer Volumenindex \\
\hline $\mathrm{EE}$ & Early Exercise \\
\hline $\mathrm{EF}$ & Ejektions-Fraktion \\
\hline EKG & Elektrokardiographie \\
\hline eNOS & Endothelial Nitric Oxide Synthase \\
\hline $\mathrm{EqO}_{2}$ & $\begin{array}{l}\text { Ventilatory Equivalent for Oxygen } \\
\text { (Atemäquivalent für Sauerstoff) }\end{array}$ \\
\hline $\mathrm{EqCO}_{2}$ & $\begin{array}{l}\text { Ventilatory Equivalent for Carbon Dioxide } \\
\text { (Atemäquivalent für Kohlendioxid) }\end{array}$ \\
\hline ER & Ejection Ratio/ Auswurfverhältnis \\
\hline ERA & Endothelin-Rezeptor-Antagonisten \\
\hline
\end{tabular}


FC

HPAH

HR

HRCT

HRP

HZV

IC

IPAH

KMR

LUFU

MAP

MPI

MRT

MVV

MW

NO

NT-proBNP

NYHA

PAH

PAP

PAPm

PAWP

$\mathrm{PCH}$

pCO2

PCWP

PDE 5-Inhibitoren

PEP

${ }_{\mathrm{PET}} \mathrm{CO}_{2}$

$\mathrm{PF}$

PFI

$\mathrm{PH}$

PVOD

PVR

\section{RAP}

$\mathrm{RC}$

RVP

RQ

SD

SI

SM

STR
Functional Class

Hereditäre pulmonal arterielle Hypertonie

Heartrate

High Resolution Computertomographie

Heart Rate Periode

Herzzeitvolumen

Index of Contractility

Idiopathische Pulmonalarterielle Hypertonie

Kardiale Magnetresonanztomografie

Lungenfunktion

Mean Arterial Pressure

Myokardialer Performance-Index (Tei-Index)

Magnetresonanztomographie

Maximal Voluntary Ventilation/ Atemgrenzwert

Mittelwert

Nitritoxid

N-Terminales Pro-Brain Natriuretic Peptide

New York Heart Association

Pulmonal arterielle Hypertonie

Pulmonary Artery Pressure

Mean Pulmonary Arterial Pressure

Pulmonary Arterial Wedge-Pressure

Pulmonary Capillary Hemangiomatosis

Partial Pressure of Carbon Dioxide

Pulmonary Capillary Wedge-Pressure

Phosphodiesterase 5-Inhibitoren

Pre-Ejection Period

End-Tidal Carbon dioxide partial Pressure

Peak Flow

Peak Flow Index

Pulmonale Hypertonie

Pulmonary Veno-Occlusive Disease

Pulmonary Vascular Resistance

Right Atrial Pressure

Recovery

Right Ventricular Pressure

Respiratory Quotient

Standard Deviation

Stroke Index

Submaximale Belastung

Systolic Time Ratio 
SV

Sono

TAPSE

TEE

TFI

TPG

TTE

TxA

UP

VAT

$\dot{\mathrm{V}} \mathrm{CO}_{2}$

$\dot{\mathrm{V} E}$

VEGF

VEPT

VET

$\dot{\mathrm{V}} \mathrm{O}_{2} \mathrm{max}$

$\dot{\mathrm{VO}}_{2} / \mathrm{HR}$

$\mathrm{VT}$

V/Q-Scan

WHO
Stroke Volume

Sonographie

Tricuspid Annular Plane Systolic Excursion

Transesophageal Echocardiography

Thoracic Fluid Index

Transpulmonaler Gradient (PAPm-PCWP)

Transthorakale Echokardiographie

Thromboxan-A

unloaded paddeling/ "Leertreten"

Ventilatory Anaerobic Threshold

Carbon Dioxide Elimination

Ventilation (Atemminutenvolumen-AMV)

Vascular Endothelial Growth Factor

Volume of Electrically Participating Tissue

(intrathoracic)

Ventricular Ejection Time

Maximal Oxygen Uptake

Oxygen Uptake/ Heart Rate (Sauerstoffpuls)

Tidal Volume

Ventilation Quotient-Scan

World Health Organisation 


\section{Zusammenfassung}

\subsection{Deutsch}

Bei Patienten mit einer pulmonalen Hypertonie wurde zur Beurteilung der linksventrikulären Funktion sowie zur Abschätzung der leistungslimitierenden Mechanismen eine fahrradergometrische Belastungsuntersuchung sowie eine nicht-invasive Messung der hämodynamischen Parameter unter Nutzung eines Bioimpedanz Monitoring-Systems durchgeführt und mit den Belastungsergebnissen der gesunden Kontrollgruppe verglichen.

Die Datenerhebung erfolgte über einen Zeitraum von 13 Monaten und schloss 24 Patienten mit pulmonaler Hypertonie und 19 gesunde Probanden ein. Das Bioimpedanz Monitoring-System ermöglicht eine hochauflösende Messung der thorakalen Impedanzänderungen und erlaubt somit die Ermittlung der Veränderungen des intrathorakalen Blutvolumens während eines Herzzyklus. Dies ermöglicht Rückschlüsse auf das Herzzeitvolumen, die Kontraktilität sowie die Flussparameter.

Die Patienten erreichten weitaus geringere Belastungsstufen als die gesunden Kontrollpersonen $(53,7 \pm 35,0$ vs. $185,4 \pm 85,4$ Watt). Weiterhin war die maximale Sauerstoffaufnahme im Patientenkollektiv deutlich reduziert im Vergleich zur Kontrollgruppe $(850,8 \pm 418$ vs. $2157,7 \pm 918 \mathrm{ml} / \mathrm{min})$. Der Anstieg der Ejektionsfraktion fiel in der Patientengruppe wesentlich geringer aus. Resultat dessen ist ein signifikant erniedrigter maximaler Herzindex bei den Patienten mit pulmonaler Hypertonie $\left(4,7 \pm 1,84\right.$ vs. $\left.8,72 \pm 4,251 / \mathrm{min} / \mathrm{m}^{2}\right)$. Unter Ruhebedingungen lag der linksventrikuläre enddiastolische Volumen-Index der Patienten unterhalb dessen der Kontrollgruppe. Während der Belastung zeigten sich jedoch in beiden Gruppen keine signifikanten Unterschiede. Der Schlagvolumen-Index war unter Ruhebedingungen bei den Patienten erniedrigt (35,63 $\pm 11,12$ vs. $\left.55,74 \pm 14,74 \mathrm{ml} / \mathrm{m}^{2}\right)$, entwickelte sich jedoch in beiden Gruppen auf unterschiedlichem Niveau parallel zueinander. In der Ejektionsphase zeigte sich unter Belastung eine deutliche

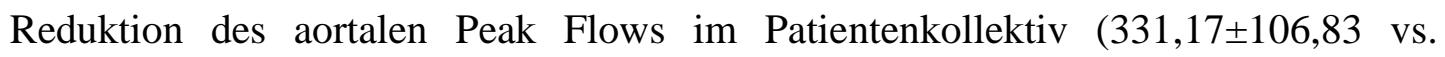
$\left.523,74 \pm 209,92 \mathrm{ml} / \mathrm{sec}^{2} / \mathrm{m}^{2}\right)$. Gleiches war bei dem Beschleunigungs-Index $\mathrm{zu}$ beobachten $\left(122,9 \pm 45,07\right.$ vs. $\left.191,07 \pm 65,05 \mathrm{sec}^{-2}\right)$. Des Weiteren war die maximale 
Herzfrequenz der Patienten im Vergleich zur Kontrollgruppe reduziert (109 \pm 18 vs. $132 \pm 30 \mathrm{~min}^{-1}$ ). Auch die weiteren hämodynamischen Parameter zeigten in Ruhe und unter Belastung keine signifikanten Unterschiede in beiden Gruppen.

Limitationen der Untersuchung liegen in der Ermittlung der Auswurfleistung und der Ventrikelvolumina. Deren Berechnung beruht auf Impedanzänderungen und Zeitintervallformeln. Dies bedeutet, dass normale Füllungsraten vermutet werden, welche bei Patienten mit einer pulmonalen Hypertonie nicht anzunehmen sind. Unberührt von diesen Gegebenheiten sind allerdings die Fluss- und Kontraktilitätsparameter, da deren Erhebung auf direkten Impedanzmessungen basiert.

Patienten mit einer pulmonalen Hypertonie sind nicht in der Lage, ihre Kontraktilität sowie Herzfrequenz im Vergleich zur Kontrollgruppe adäquat zu steigern. Es zeigte sich unter Ruhe sowie unter Belastung eine Reduktion der linksventrikulären Funktion. Des Weiteren lassen die reduzierten Inotropie-Indizes auf eine globale kardiale Beeinträchtigung des Herzens schließen, welche über eine Rechtsherzbelastung hinauszugehen scheint. Bemerkbar macht sich dies, dass bei Steigerung der Inotropie der Sauerstoffpuls, welcher als Äquivalent des Schlagvolumens angesehen wird, nicht in gleichem Maße ansteigt wie bei Gesunden. Mögliche Ursachen dieser hämodynamischen Veränderungen stellen die Entwicklung eines septalen Shifts sowie eine Asynchronie, bedingt durch die veränderten Druckverhältnisse unter Belastung, dar und sind Ziel weiterer Untersuchungen.

\subsection{Englisch}

To assess left ventricular cardiac performance and to estimate the performancelimiting mechanisms in patients with pulmonary hypertension, we performed cardiopulmonary exercise testing by bicycle-ergometer and a non-invasive measurement of hemodynamic parameters using a bioimpedance-monitoring system and compared the results with the healthy control group. 24 patients with pulmonary hypertension and 19 healthy volunteers were included over a period of 13 months.

The bioimpedance-monitoring system offers a high frequency measurement of thoracic impedance changes and allows the determination of changes in intrathoracic blood volume during a cardiac cycle. This enables conclusions of the cardiac output, the contractility and the flow parameters. 
Patients with pulmonary hypertension tolerated less workload compared to the healthy control group $(53,7 \pm 35,0$ vs. $185,4 \pm 85,4$ watt $)$. Furthermore the maximal oxygen uptake was reduced in patients compared to control $(850,8 \pm 418$ vs. $2157,7 \pm 918 \mathrm{ml} / \mathrm{min})$. The increase of the ejection fraction in patients was considerably lower. The result was a significantly decreased maximal cardiac index in patients with pulmonary hypertension $\left(4,7 \pm 1,84 \mathrm{vs} .8,72 \pm 4,251 / \mathrm{min} / \mathrm{m}^{2}\right)$. The left ventricular end-diastolic volume index at rest was lower in patients than in the control group. During exercise, there was no significant difference in both groups. The stroke index was markedly reduced in patients $(35,63 \pm 11,12$ vs. $55,74 \pm 14,74 \mathrm{ml} / \mathrm{m}^{2}$ ) during resting conditions, but increased in both groups in parallel at different levels. The aortic peak-flow during left ventricular ejection was decreased in patients during exercise $\left(331,17 \pm 106,83\right.$ vs. $\left.523,74 \pm 209,92 \mathrm{ml} / \mathrm{sec} / \mathrm{m}^{2}\right)$ as well as the acceleration index $\left(122,9 \pm 45,07\right.$ vs. $\left.191,07 \pm 65,05 \mathrm{sec}^{-2}\right)$. In addition, the maximal heart rate was lower in patients compared to the control group $(109 \pm 18$ vs. $132 \pm 30 \mathrm{~min}^{-1}$ ). The remaining hemodynamic parameters showed no significant differences between both groups at rest and during exercise testing.

Limitations of the examination can be found in the determination of cardiac output and the ventricular volume. Their evaluation is based on impedance changes and time interval formulas, which assume normal filling rates. This is not necessarily the case in patients with pulmonary hypertension.

The parameters of flow- and contractility are unaffected by this conditions, because their calculation is based on direct impedance measurements.

In summary, patients with pulmonary hypertension were not able to compensate the lack of contractility by increasing heart rate in contrast to the control group. At rest and during exercise a reduction of left ventricular performance was found.

Furthermore, the reduced inotropic indices suggest a global impairment of the heart, which seems to go beyond right ventricular dysfunction. It is shown that by increase of inotropy the oxygen pulse, which is considered as the equivalent of stroke volume, is not increased as much as in healthy subjects.

Possible explanations of these hemodynamic changes are the development of a septal shift and asynchrony, due to changed pressure under increased workload. These effects are target of future investigations. 


\section{Einleitung}

Die pulmonale Hypertonie ist eine schwerwiegende Erkrankung, die bei betroffenen Patienten zur Einschränkung der Belastbarkeit und Lebensqualität führt und unbehandelt mit einer schlechten Prognose behaftet ist.

Eines der ersten und häufigsten Symptome, aufgrund derer sich die Patienten bei einem Arzt vorstellen, ist die Belastungsdyspnoe. Unter einem Lungenhochdruck leidet jedoch nur die Minderheit. Nach Ausschluss der häufigen respiratorischen Störungen oder kardiovaskuläre Erkrankungen sollte insbesondere bei Vorliegen entsprechender Risikofaktoren (rezidivierende Embolien, Appetitzügler, familiäre Disposition) auch an eine pulmonale Hypertonie gedacht werden, insbesondere beim Auftreten von Synkopen, Angina pectoris oder bei progressiven Einschränkungen der Belastungskapazität. Bedingt durch die unspezifischen Symptome, wie Belastungsdyspnoe, Müdigkeit, Vermeidung körperlicher Anstrengung wird die pulmonale Hypertonie oft erst spät erkannt (Tabelle 1). Die Diagnosestellung erfolgt oft erst bei manifesten Zeichen einer Rechtsherzinsuffizienz. Leider dauert es meist mehrere Monate bis Jahre bis zur Vorstellung der Patienten in einer Spezialambulanz (Olschewski, Hoeper et al. 2006). Durchschnittlich vergehen 2,5 Jahre vom Auftreten der ersten Symptome bis zur Diagnosestellung (Wilkens, Grimminger et al.).

Das Problem liegt darin, mit einem klinisch vertretbaren diagnostischen Aufwand tatsächlich erkrankte Patienten frühzeitig zu erkennen ohne Gesunde einer unnötigen Untersuchung zu unterziehen und diese somit zu beunruhigen oder gar durch die Untersuchung zu gefährden.

\begin{tabular}{|l|c|c|}
\hline \multicolumn{2}{|l|}{ Symptome der pulmonalen Hypertonie nach Häufigkeiten } \\
\hline & Erstsymptom & bei Diagnosestellung \\
\hline Luftnot & $60 \%$ & $98 \%$ \\
\hline Müdigkeit & $19 \%$ & $73 \%$ \\
\hline Thorakale Beschwerden & $7 \%$ & $47 \%$ \\
\hline Schwächeanfälle & $5 \%$ & $41 \%$ \\
\hline Beinödeme & $3 \%$ & $37 \%$ \\
\hline Palpitationen & $5 \%$ & $33 \%$ \\
\hline
\end{tabular}

Tabelle 1: Symptome der pulmonalen Hypertonie (Rich, Dantzker et al. 1987)

Ein lebenslimitierender Faktor ist die durch den erhöhten Lungenwiderstand entstehende chronische Rechtsherzinsuffizienz. Des Weiteren kommt es aufgrund der chronischen Unterfüllung des linken Ventrikels $\mathrm{zu}$ einer Reduktion der 
linksventrikulären Funktion. Die daraus resultierenden hämodynamischen Veränderungen führen $\mathrm{zu}$ einer Einschränkung der Belastbarkeit und der Leistungsfähigkeit bei den betroffenen Patienten. Noch bis vor einigen Jahren war die Lungentransplantation die einzige therapeutische Möglichkeit, da noch keine wirksamen medikamentösen Mittel zur Verfügung standen. Seitdem hat sich jedoch viel verändert, so dass bis heute mehrere Medikamente zur Behandlung der pulmonalen Hypertonie zur Verfügung stehen.

\subsection{Historisches zur pulmonalen Hypertonie}

Die pulmonale Hypertonie wurde erstmals 1891 durch den deutschen Professor Ernst von Romberg beschrieben. Bevor es möglich war, den pulmonalarteriellen Druck invasiv zu bestimmen, galt die pulmonale Arteriosklerose als weitgehend akzeptierter Nachweis für eine chronische pulmonal arterielle Hypertonie (Romberg 1891). Im Jahr 1891 stufte Ernst von Romberg diese Erkrankung als „Sklerose der Lungenarterie“ ein, da er zu diesem Zeitpunkt nicht in der Lage war, die Ursache dieser pulmonalvaskulären Läsion festzustellen. Dr. Abel Ayerza, Professor an der Universität von Buenos Aires, beschrieb 1901 in seinen Vorlesungen die klinischen Kennzeichen dieser Erkrankung. Er erkannte, dass die klinischen Symptomen wie Dyspnoe, chronische Zyanose und Polyzythämie im Zusammenhang mit der durch die Autopsie gesicherten Diagnose einer Lungenarteriensklerose standen. Einer seiner Studenten, Arrillaga, bezeichnete dieses Syndrom nachträglich als „Ayerza's Krankheit“. Im Jahre 1935 lokalisierte Oscar Brenner die kleinen muskulären Arterien und Arteriolen als Ursprung der pulmonalen Hypertension, verkannte jedoch, welche funktionelle Rolle die Vasokonstriktion in der Pathogenese spielt. Weiterhin war ihm der kausale Zusammenhang zwischen pulmonaler Gefäßläsion und der Hypertrophie des rechten Herzens nicht bewusst. Erst im späten zwanzigsten Jahrhundert erkannten Physiologen den Zusammenhang zwischen Beiden (Hill 2008).

\subsection{Klassifikation der pulmonalen Hypertonie}

Seit der ersten WHO-Weltkonferenz zur pulmonalen Hypertonie im Jahre 1973 erfolgte eine Einteilung in primäre und sekundäre pulmonale Hypertonie. Diese wurde bis zu der WHO-Weltkonferenz 1998 beibehalten. Damals wurde eine diagnostische Klassifikation mit der Differenzierung in fünf Krankheitsgruppen 
vorgeschlagen. Diese Klassifikation wurde auf den WHO-Weltkonferenzen in Venedig 2003 und Dana Point 2008 weiterentwickelt (siehe Tabelle 2).

In der Gruppe 1 (pulmonal arterielle Hypertonie) finden sich die idiopathische pulmonale Hypertonie (IPAH), die erbliche/ hereditäre PAH (früher familiäre pulmonale Hypertonie), PH bei kongenitalen Herzfehlern als auch die assoziierte PAH beispielsweise HIV-Infektionen oder Kollagenosen. Die pulmonale venookklusive Erkrankung sowie die pulmonal-kapilläre Hämangiomatose teilen einige Eigenschaften der PAH, weisen jedoch auch etliche Unterschiede auf. Dies ist der Grund, weshalb man sich entschied diese Krankheitsbilder in eine eigene Kategorie einzuordnen. Eine vollständige Trennung von der PAH erschien nicht sinnvoll, daher die Bezeichnung als klinische Untergruppe 1`. Gruppe 2 erfasst die pulmonale Hypertonie bei Linksherzerkrankungen. In der Gruppe 3 befinden sich die pulmonale Hypertonie bei Lungenerkrankungen und Hypoxie, zum Beispiel bei interstitiellen Lungenerkrankungen oder chronischen Lungenerkrankungen wie die COPD. Zur Gruppe 4 gehört die chronisch-thromboembolische pulmonale Hypertonie. Abschließend sind in Gruppe 5 Formen der pulmonalen Hypertonie mit unklaren oder multifaktoriellen Ursachen aufgeführt (Galie, Hoeper et al. 2009). 
klinische Klassifikation der pulmonalen Hypertonie

1. Pulmonal-arterielle Hypertonie (PAH)

1.1 Idiopathisch (IPAH)

1.2 Hereditär (HPAH)

1.2.1 BMPR2-Mutation

1.2.2 ALK1-, Endoglin- Mutation, (mit und ohne hämorrhagische Teleangiektasie)

1.2.3 Unbekannte Mutationen

1.3 Medikamenten- oder drogeninduziert

1.4 Assoziiert mit (APAH)

1.4.1 Kollagenosen

1.4.2 HIV- Infektion

1.4.3 Portale Hypertension

1.4.4 Kongenitale Herzerkrankungen

1.4.5 Schistosomiasis

1.4.6 Chronische hämolytische Anämie

1.5 Persistierende pulmonale Hypertonie bei Neugeborenen

1 Pulmonale venookklusive Erkrankungen (PVOD) und pulmonal-kapilläre Hämangiomatose ( $\mathrm{PCH})$

2. Pulmonale Hypertonie bei Linksherzerkrankungen

2.1 systolische Herzinsuffizienz

2.2 diastolische Herzinsuffizienz

3. Pulmonale Hypertonie bei Lungenerkrankungen und/ oder Hypoxie

3.1 chronisch-obstruktive Lungenerkrankungen

3.2 Interstitielle Lungenerkrankungen

3.3 Andere Lungenkrankheiten mit gemischter Ventilationsstörung

3.4 Schlafassoziierte Atmungsstörungen

3.5 Alveoläre Hypoventilation

3.6 Chronischer Aufenthalt in Höhenlagen

3.7 Pulmonale Entwicklungsstörungen

4. Chronisch-thromboembolische pulmonale Hypertonie (CTEPH)

5. pulmonale Hypertonie bei unklarer oder multifaktorieller Ursache

5.1 Hämatologischer Erkrankungen

5.2 Systemerkrankungen (Sarkoidose, Langerhanszellgranulomatose, Neurofibromatose, Vaskulitis)

5.3 Metabolische Erkrankungen (Glykogenspeicherkrankheiten, M.Gaucher, Schilddrüsenerkrankungen)

5.4 Sonstige (tumorbedingte Obstruktion, fibrosierende Mediastinitis, chronisches Nierenversagen mit Dialyse)

ALK-1: Actin-receptor-like Kinase

BMPR2: Bone morphogenetic protein receptor 2

APAH: associated pulmonary arterial hypertension

Tabelle 2: klinische Klassifikation der pulmonalen Hypertonie (Galie, Hoeper et al. 2009)

\subsection{Epidemiologie}

Schätzungen zufolge liegt die Prävalenz der idiopathischen PAH (IPAH) in der westlichen Welt (Industrieländern) bei sechs pro einer Million und die der pulmonalen arteriellen Hypertonie bei 15 pro einer Million (Humbert, Sitbon et al. 2006). Frauen sind von der IPAH fast doppelt so häufig betroffen wie Männer (Gaine and Rubin 1998). Genauere Prävalenzdaten der anderen Formen der pulmonalen Hypertonie liegen noch nicht vor.

\subsection{Pathogenese}

Die pulmonale Hypertonie hat eine multifaktorielle Pathogenese. Es gibt zahlreiche Trigger-Mechanismen sowie genetische Dispositionen, wie beispielsweise die BMPR2-, ALK1- und 5HTT-Mutationen, die bei einem kleinen Prozentsatz zu einer Manifestation der Erkrankung führen können. Grundlegend lässt sich sagen, dass es 
sich bei der pulmonalen Hypertonie um eine chronische Erkrankung der Lungengefäßstrombahn handelt, die alle drei Schichten der Gefäßwand - Intima, Media und Adventitia - betrifft. Die Erhöhung des pulmonalarteriellen Druckes aufgrund der Verengungen des Gefäßvolumens und die damit verbundene Erhöhung des Widerstandes sind durch die Intima- und Mediaverdickungen verursacht (Gaine and Rubin 1998; Rubin 2002).

Die Verdickung der Adventitia bewirkt einen Abfall der Compliance und Dehnbarkeit der Gefäße, was wiederum zu einem weiteren Druckanstieg führt. Daraus resultiert eine Zellschädigung und es kommt zu der Entwicklung einer endothelialen Dysfunktion aufgrund der Beeinträchtigung der metabolischen Eigenschaften des Gefäßendothels, bedingt durch die morphologischen und funktionellen Veränderungen (Rosenkranz 2008).

Die Vasokonstriktion in der Lungenstrombahn entsteht durch die Imbalance zwischen vasodilatativen (Prostazyklin, NO) und vasokonstriktiven (Endothelin, Thromboxan-A, Vascular Endothelial Growth Factor) Mediatoren (siehe Abbildung 1).

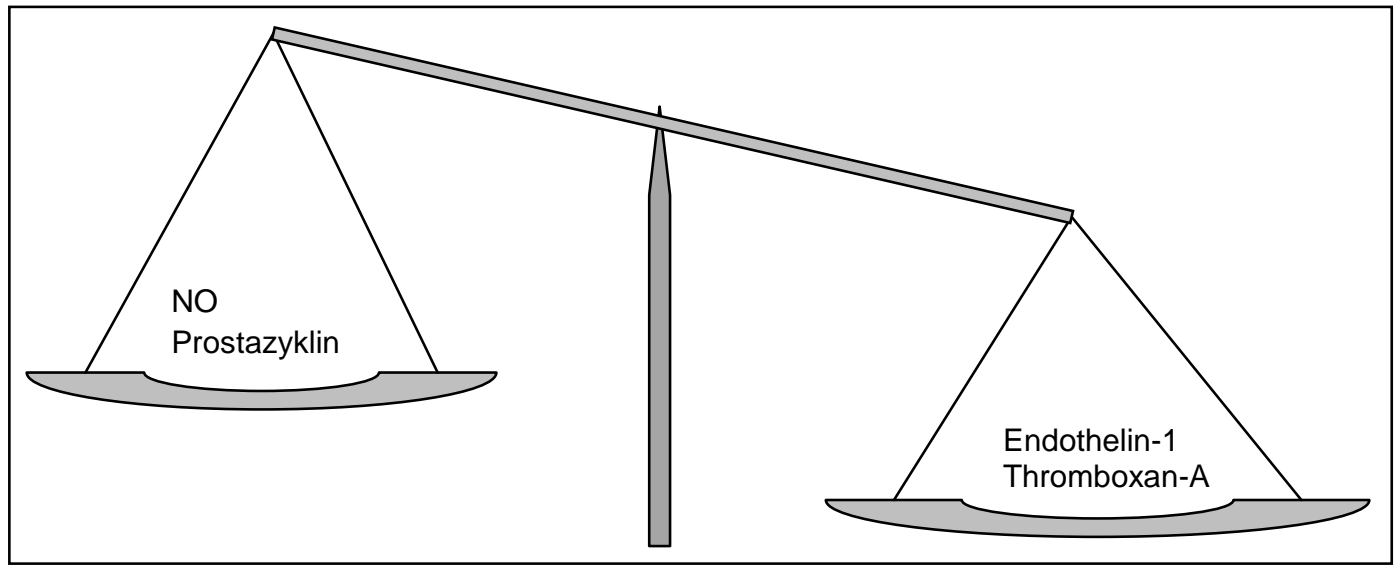

Abbildung 1: Imbalance zwischen vasodilatativen und vasokonstriktiven Mediatoren

Die verringerte Synthese vasodilatierender Substanzen ist durch die herabgesetzte Expression endothelialer Stickstoffmonoxidsynthetase (eNOS) und Prostazyklinsynthetase bedingt (Christman, McPherson et al. 1992; Giaid and Saleh 1995).

Des Weiteren scheint das vaskuläre Remodeling in der Pathogenese eine entscheidende Rolle zu spielen. Die hierbei entstehenden proliferativen Veränderungen der Gefäßwand führen zur Einwanderung von 
Entzündungsmediatoren, wie Makrophagen und T- und B-Lymphozyten (Hassoun, Mouthon et al. 2009; Morrell, Adnot et al. 2009).

Charakteristischerweise lassen sich eine konzentrische oder exzentrische Fibrose der Intima einhergehend mit plexiformen Läsionen sowie eine Hypertrophie der Media als auch der Adventitia beobachten. Diese charakteristischen Veränderungen der Gefäßwand entstehen als Folge einer erhöhten Proliferationsrate sowie einer reduzierten Apoptoserate der glatten Gefäßmuskelzellen. Folge dessen ist eine gesteigerte Muskularisierung vor allem der kleinen Pulmonalarterien mit zunehmender Reduzierung des Gefäßlumens. Die endotheliale Schädigung hat eine reduzierte flussabhängige Dilatation der Gefäße aufgrund verminderter Ausschüttung der entsprechenden Mediatoren zur Folge. Diese Effekte werden durch die verlängerte Diffusionsstrecke und Fibrose weiter reduziert. Dies führt zu einem Anstieg des pulmonal-kapillären Druckes sowie zu einem erhöhten pulmonal vaskulären Widerstand. Belastungsbedingungen führen $\mathrm{zu}$ einem progredienten Anstieg des pulmonal arteriellen Druckes, da eine Kompensation des vermehrten pulmonalen Blutflusses über Vasodilatation nicht mehr gewährleistet ist (Erdmann 2011).

Aktuelle Therapien zielen darauf ab, das Ungleichgewicht der vasoaktiven Mediatoren durch die Hemmung vasokonstriktiver Signalstoffe (EndothelinrezeptorAntagonisten) und Verstärkung der vasodilatativen NO- (Phosphodiesterase-5Inhibitoren z.B.Sildenafil) und Prostazyklin-Signalwege (z.B. Iloprost) aufzuheben (Humbert, Sitbon et al. 2004). Leider bewirkt die Therapie keine wesentliche Beeinflussung der grundlegenden morphologischen Gefäßwandveränderungen. Jedoch scheint es zu einer temporären Verbesserung der Hämodynamik als auch der klinischen Symptomatik sowie zu einer Abschwächung der Krankheitsprogression zu kommen (Rosenkranz 2008).

\subsection{Beurteilung des Schweregrades der Erkrankung}

Ein weiterer wichtiger Faktor zur Beurteilung des Patienten ist dessen funktioneller Schweregrad. Dieser ermöglicht prognostische Einschätzungen und hat Bedeutung für etwaige therapeutische Entscheidungen. Seit der Evian-Konferenz 1998 gilt hierfür eine modifizierte NYHA-Klassifikation (Tabelle 3), welche 2003 in der Venedig-Konferenz ohne Änderungen übernommen wurde (Barst, McGoon et al. 2004). Die Anwendung ist jedoch bei dem Vorliegen von Begleiterkrankungen wie 
beispielsweise bei schweren rheumatischen Erkrankungen schwierig. Gleiches gilt für sehr alte und gebrechliche Patienten als auch für Kinder unter dem 8. Lebensjahr (Olschewski, Hoeper et al. 2006).

\begin{tabular}{|l|l|}
\hline Funktionelle Klassifikation der pulmonalen Hypertonie \\
\hline Klasse I & $\begin{array}{l}\text { Patienten mit einer PHT ohne Einschränkung der normalen körperlichen } \\
\text { Belastbarkeit. Eine normale körperliche Aktivität führt nicht zu zunehmender } \\
\text { Dyspnoe, Müdigkeit, thorakalen Schmerzen oder Synkopen. }\end{array}$ \\
\hline Klasse II & $\begin{array}{l}\text { Patienten mit einer PHT mit leichten Einschränkungen in der körperlichen Aktivität. } \\
\text { Keine Beschwerden unter Ruhebedingungen. Eine normale körperliche Belastung } \\
\text { führt zu der Zunahme von Dyspnoe, Müdigkeit sowie thorakalen Beschwerden und } \\
\text { Synkopen. }\end{array}$ \\
\hline Klasse III & $\begin{array}{l}\text { Patienten mit einer pulmonalen Hypertonie und deutlichen Einschränkungen in der } \\
\text { körperlichen Belastbarkeit. Keine Beschwerden unter Ruhebedingungen. Bereits } \\
\text { leichtere Aktivitäten führen zu der Zunahme von Dyspnoe, Müdigkeit, thorakalen } \\
\text { Beschwerden sowie Synkopen. }\end{array}$ \\
\hline Klasse IV & $\begin{array}{l}\text { Patienten mit einer pulmonalen Hypertonie, welche nicht in der Lage sind } \\
\text { irgendwelche körperlichen Aktivitäten ohne Beschwerden in Ruhe auszuführen. Es } \\
\text { können Zeichen einer manifesten Rechtsherzinsuffizienz vorliegen. Müdigkeit und/ } \\
\text { oder Dyspnoe können bereits in Ruhe auftreten. Es kommt bei nahezu jeder } \\
\text { körperlichen Belastung zu einer Zunahme der Beschwerden. }\end{array}$ \\
\hline
\end{tabular}

Tabelle 3: WHO-Klassifikation des funktionellen Schweregrades bei Patienten mit pulmonaler Hypertonie (Barst, McGoon et al. 2004)

Ein weiteres Hilfsmittel zur Einschätzung des Schweregrades der Erkrankung ist die Borg-Skala. Sie dient der Beurteilung des subjektiven Empfindens der Atemnot nach einem Belastungstest, wie beispielsweise nach einer spiroergometrischen Untersuchung. Die seit 1982 verwendete „modifizierte Borg-Skala“ erlaubt die Einteilung in zwölf Stufen bei einem Minimum von 0 und Maximum von 10 (siehe Tabelle 4). Hauptsächliche Verwendung liegt in der subjektiven Einschätzung von Schmerzen sowie Atemnot (Borg 1982). 


\begin{tabular}{|c|c|}
\hline & empfundene Dyspnoe \\
\hline $\mathbf{0}$ & keine \\
\hline $\mathbf{0 , 5}$ & gerade wahrnehmbar \\
\hline $\mathbf{1}$ & sehr leicht \\
\hline $\mathbf{2}$ & leicht \\
\hline $\mathbf{4}$ & moderat \\
\hline $\mathbf{5}$ & schwer \\
\hline $\mathbf{6}$ & \\
\hline $\mathbf{7}$ & \\
\hline $\mathbf{8}$ & sehr, sehr schwer \\
\hline $\mathbf{9}$ & maximal \\
\hline $\mathbf{1 0}$ & Tabelle 4: modifizierte Borg-Skala \\
\hline \multicolumn{2}{|c|}{} \\
\hline
\end{tabular}

\subsection{Diagnostik}

Die pulmonale Hypertonie $(\mathrm{PH})$ ist charakterisiert durch eine Erhöhung des pulmonalarteriellen Mitteldruckes auf über $25 \mathrm{mmHg}$ unter Ruhebedingungen, gemessen mittels Rechtsherzkatheter. Da die ersten Symptome der pulmonalen Hypertonie unspezifisch sind und sich erheblich mit weit häufigeren Krankheitsbildern überschneiden, ist eine frühe Diagnose erschwert und fokussiert auf eine primär nicht-invasive Diagnostik. Als Basisdiagnostik ist daher eine Lungenfunktionsprüfung, Belastungsuntersuchung und allgemeine Echokardiografie zu fordern.

Wurde hier der Verdacht auf das Vorliegen einer PH geäußert, so sollte eine gezielte echokardiografische Beurteilung des rechten Ventrikels und der rechtsseitigen Hämodynamik erfolgen. Dies ermöglicht eine Differenzierung, ob eine Linksherzoder Lungenerkrankung ursächlich für die pulmonale Hypertonie ist. Kann dies ausgeschlossen werden oder existieren Hinweise auf eine «out-of-proportion» $\mathrm{PH}$, welche durch die Grunderkrankung allein nicht erklärbar sind, sollte eine systematische Abklärung erfolgen. Weitere zu berücksichtigende Merkmale sind die funktionalen sowie hämodynamischen Einschränkungen als auch die Einbeziehung der Ätiologie (Wilkens 2010).

Die Abgrenzung zu Lungen- und Linksherzerkrankungen erfolgt mittels EKG, Lungenfunktion, Röntgenbild und Computertomographie des Thorax. Sollten diese Untersuchungen keinen wegweisenden Befund liefern, wird die Durchführung einer Ventilations-Perfusionsszintigraphie empfohlen. Dies ermöglicht das Herausfiltern 
der Patienten, welche an einer chronisch-thromboembolischen pulmonalen Hypertonie (CTEPH) leiden und daher eventuell operiert werden können. Die Patienten benötigen eine Pulmonalis-Angiografie als zusätzliche Diagnostik. Im nächsten Schritt erfolgt die Durchführung eines Rechtsherzkatheters. Letzteres sollte nach Möglichkeit in einem Zentrum für pulmonale Hypertonie durchgeführt werden. Im Folgenden wird auf die wichtigsten invasiven und nicht-invasiven Methoden separat eingegangen.

\subsubsection{EKG}

Bei Patienten mit einer leichten oder latenten pulmonalen Hypertonie finden sich im Ruhe-EKG meist keine spezifischen Auffälligkeiten, wohingegen in $83 \%$ der Fälle bei einer behandlungsbedürftigen pulmonalen Hypertonie differenzierte pathologische Befunde zu finden sind (Olschewski, Hoeper et al. 2006). Bei den PAH Patienten beträgt die Inzidenz supraventrikulärer Tachykardien, welche zu einer Verschlechterung der Klinik sowie einer rechtskardialen Dekompensation führen kann, etwa $3 \%$ pro Jahr (Tongers, Schwerdtfeger et al. 2007).

\begin{tabular}{|c|c|}
\hline \multicolumn{2}{|c|}{ EKG-Befunde bei Rechtsherzbelastung } \\
\hline 1 & $\begin{array}{l}\text { Herzthythmusstörungen } \\
\text { - Vorhofflimmern/ -flattern } \\
\text { - } \quad \text { ektope SVES/ VES } \\
\end{array}$ \\
\hline 2 & $\begin{array}{l}\text { P-pulmonale/ P- dextroatriale } \\
\text { P- Welle in II > 0,25 mm }\end{array}$ \\
\hline 3 & Inkompletter oder kompletter Rechtsschenkelblock \\
\hline 4 & $\begin{array}{l}\text { Abweichung der Herzachse nach rechts ggf. Sagittalstellung } \\
\left(S_{I} Q_{I I I} \text { oder } S_{I} S_{I I} S_{I I I}-T y p\right)\end{array}$ \\
\hline 5 & $\begin{array}{l}\text { Rechtsherzhypertrophiezeichen } \\
\text { - } \mathrm{R} \text { in } \mathrm{V}_{1} / \mathrm{V}_{2}>0,7 \mathrm{mV} \\
\text { - } \mathrm{S} \text { in } \mathrm{V}_{5} / \mathrm{V}_{6}>0,7 \mathrm{mV} \\
\text { - } \mathrm{R}>\mathrm{S} \text { in } \mathrm{V} 1 \\
\text { - } \text { Sokolow- Lyon- Index: } \mathrm{R} \text { in } \mathrm{V}_{1}+\mathrm{S} \text { in } \mathrm{V}_{5} \text { oder } \mathrm{V}_{6}>1,05 \mathrm{mV}\end{array}$ \\
\hline 6 & $\begin{array}{l}\text { Rechts-präkordiale Repolarisationsstörungen } \\
\text { ST-Streckenveränderungen (deszendierende) meist in } \mathrm{V}_{2}-\mathrm{V}_{4} \text { sowie } \\
\text { horizontale ST-Senkungen in II, III, aVF }\end{array}$ \\
\hline
\end{tabular}

\section{Tabelle 5: EKG-Befunde bei Rechtsherzbelastung}

Das problematische an der Diagnostik der pulmonalen Hypertonie sind die unspezifischen Frühsymptome. Das EKG ist die am breitesten verfügbare und die am häufigsten durchgeführte Untersuchung bei Belastungsintoleranz oder Thoraxschmerz. Dies ist ein Grund weshalb auf den Wert der EKG-Beurteilung 
zunehmendes Augenmerk gelegt wird, insbesondere auf Zeichen der Rechtsherzbelastung (siehe Tabelle 5) zur frühen Identifizierung einer präkapillären pulmonalen Hypertonie (Speich 2011). In einer Studie von Fuster et al. in den 80er Jahren wurden bereits bei $95 \%$ der IPAH-Patienten EKG-Veränderungen, wie eine rechtsventrikuläre Hypertrophie, eine nach rechtsabweichende Herzachse sowie ein P-pulmonale beobachtet (Fuster, Steele et al. 1984). Mit der Entwicklung eines nicht-invasiven Diagnose-Algorithmus zur Identifizierung oder Ausschluss einer präkapillären $\mathrm{PH}$ beschäftigten sich Bonderman et al. Hierbei stellten sich die Zeichen einer Rechtsherzbelastung als stärkste Prädiktoren für eine präkapilläre $\mathrm{PH}$ heraus (Bonderman, Wexberg et al. 2011).

\subsubsection{Röntgen Thorax in zwei Ebenen}

Die sichtbaren Veränderungen in der Thoraxröntgenaufnahme stehen im Zusammenhang mit der Krankheitsdauer und der Krankheitsgenese der pulmonalen Hypertonie. Ist in der Übersichtsaufnahme (p.a. Strahlengang) beispielsweise eine Rechtsherzvergrößerung sowie eine verbreiterte Herzsilhouette zu erkennen, ist dies ein Hinweis auf eine chronische Belastung des rechten Herzens. Darüber hinaus ist eine verlängerte sternale Kontaktfläche im Seitenbild charakteristisch. Des Weiteren sind dilatierte zentrale Pulmonalarterien (Truncus intermedius rechts $>16 \mathrm{~mm}$ ) als auch ein dilatiertes Pulmonalissegment sowie eine Rarefizierung der Gefäße in der Peripherie kennzeichnend für eine chronische pulmonale Hypertonie. Obwohl die Röntgenaufnahme des Thoraxes bei einem Großteil der PAH-Patienten bereits zum Diagnosezeitpunkt Pathologien aufweist, führt ein unauffälliges Röntgenbild jedoch keinesfalls zum Ausschluss einer pulmonalen Hypertonie (Olschewski, Hoeper et al. 2006). Zusätzlich erlaubt es eine Beurteilung des Lungenparenchyms und gibt Hinweise auf eine Linksherzvergrößerung, ein Emphysem, das Vorliegen einer Stauung oder eines Ergusses (Komplikation).

\subsubsection{6-Minuten-Gehtest}

Der Sechs-Minuten-Gehtest (6-MWT) ist ein guter Parameter der kardiopulmonalen Leistungsfähigkeit des Patienten. Er hat als Prognoseparameter große Bedeutung und wurde zur Therapiesteuerung etabliert (Miyamoto, Nagaya et al. 2000). Es erfolgt die Messung der Strecke in Metern, welche der Patient in einem für ihn angenehmen Tempo innerhalb von sechs Minuten zurücklegt. Unter Berücksichtigung individueller Unterschiede gilt eine Strecke von $400 \mathrm{~m}$ beziehungsweise $500 \mathrm{~m}$ 
(jüngere Patienten) als zufriedenstellend (Galie, Hoeper et al. 2009). Entspricht die Gehstrecke weniger als 332 m oder liegt eine Sauerstoffentsättigung von >10\% vor, so ist dies hinweisend auf eine eingeschränkte Prognose bei Patienten mit einer pulmonalen Hypertonie (Miyamoto, Nagaya et al. 2000) (Paciocco, Martinez et al. 2001). Die Aussagekraft dieses Testes ist jedoch bedingt durch Alter, Geschlecht, Körpergröße und -gewicht sowie Komorbiditäten eingeschränkt (2002). Normwerte existieren nicht und die unterschiedlichen Grenzwerte sind nicht für alle Formen der pulmonalen Hypertonie in dieser Art validiert. Gleiches gilt für die unter Therapie auftretenden Veränderungen.

\subsubsection{Lungenfunktion und Blutgasanalyse}

Die Bodyplethysmographie eignet sich zur Differenzierung von Patienten mit normaler Lungenfunktion und Patienten mit obstruktiven oder restriktiven Ventilationsstörungen, die ursächlich für eine pulmonale Hypertonie sein können. Die Lungenfunktionsuntersuchung zeigt bei PAH-Patienten meist eine leicht eingeschränkte Diffusionskapazität sowie eine geringfügige kombinierte Ventilationsstörung, oft ist sie jedoch auch völlig normal. Die Diffusionskapazität für Kohlenmonoxid $\left(\mathrm{DL}_{\mathrm{CO}}\right)$ ist bei einer pulmonal arteriellen Hypertonie im Mittel auf circa $60-80 \%$ des Sollwertes reduziert. Hinweisend auf eine Lungenbeteiligung einer Kollagenose beziehungsweise eine primäre Lungenerkrankung sind Werte unterhalb von $50 \%$ des Solls (Wilkens 2010). In der arteriellen Blutgasanalyse zeigt sich meist eine mäßige Hypoxämie. Aufgrund der Hyperventilation kommt es zu einem erniedrigten $\mathrm{pCO}_{2}$ (Hoeper, Pletz et al. 2007). Letztendlich ist die Bewertung der Blutgasanalyse abhängig von vielen Faktoren einerseits von der korrekten Durchführung bei dyspnoeischen Patienten andererseits vom HZV, von Shunts, von der Diffusionsbarriere dem $\mathrm{Hb}$, als auch der zur Verfügung stehenden ventilatorischen Oberfläche.

\subsubsection{Spiroergometrie}

Bei einer vorliegenden Belastungsdyspnoe liefert die Durchführung einer Spiroergometrie wichtige Hinweise zur Diagnosestellung. Die Früherkennung einer pulmonalen Hypertonie wird mittels charakteristischer Gasausstauschmuster erleichtert (Olschewski, Hoeper et al. 2006; Hansen, Ulubay et al. 2007). Ist bereits die Diagnose einer pulmonalen Hypertonie gestellt, so stellt die Spiroergometrie eine unverzichtbare diagnostische Möglichkeit zur nicht-invasiven Beurteilung des 
Schweregrades, der Prognose sowie zur Beurteilung des Krankheitsverlaufs dar. Wichtige Parameter zur Beurteilung der am Gasaustausch beteiligten Organe sind die maximale Sauerstoffaufnahme $\left(\dot{\mathrm{V}}_{2} \mathrm{max}\right)$, die Kohlendioxidabgabe als auch die Ventilation. Eine maximale Sauerstoffaufnahme kleiner $10,4 \mathrm{ml} / \mathrm{min} / \mathrm{kg}$ stellt eine prognostisch ungünstige Konstellation dar (Dumitrescu and Rosenkranz 2008). Der Sauerstoffpuls $\left(\mathrm{V}_{2} / \mathrm{HR}\right)$ ist bei Patienten mit einer pulmonalen Hypertonie vermindert. Als Normwert ist ein Sauerstoffpuls von mehr als $10 \mathrm{ml}$ pro Herzschlag unter maximaler Belastung anzusehen (Wonisch 2003). Der endexpiratorische $\mathrm{CO}_{2-}$ Partialdruck $\left(\mathrm{PETCO}_{2}\right)$ ist bei den Patienten unter Belastung erniedrigt (Yasunobu, Oudiz et al. 2005). Ein weiterer Effekt welcher bei Patienten mit pulmonaler Hypertonie zu beobachten ist, ist ein im Vergleich zu Gesunden steilerer Anstieg der Beziehung von Ventilation versus Kohlendioxidabgabe ( $\dot{\mathrm{VE}} / \dot{\mathrm{VCO}}_{2}$-Slope) (Dumitrescu, Oudiz et al. 2010). Die Sauerstoffsättigung sowie das Verhalten des Blutdruckes liefern weitere wichtige Informationen. Prognostisch ungünstig ist beispielsweise ein reduzierter maximaler Blutdruckanstieg (systolisch kleiner 120 $\mathrm{mmHg}$ ) unter Belastungsbedingungen (Dumitrescu, Oudiz et al. 2010).

\subsubsection{Blutdiagnostik und Immunologie}

Die Bestimmung laborchemischer Routineparameter (Hämatologie, Schilddrüsenfunktionstest) sowie spezieller Laboruntersuchungen sollte bei allen Patienten mit pulmonaler Hypertonie erfolgen. Dies hilft unter anderem, eine eventuell zugrunde liegende Kollagenose, Hepatitis- oder HIV-Erkrankung zu erkennen (Grünig, Barner et al. 2010). Bei Patienten mit idiopathischer pulmonaler Hypertonie weisen bis zu $40 \%$ positive anti-nukleäre Antikörper (ANA) auf, mit Titern von mindestens 1:80 (Rich, Kieras et al. 1986). Zur Differenzierung der Kollagenosen tragen Parameter wie Centromer- und Anti-Ro, Scl-70, U1- und U3RNP-Antikörper sowie anti-ds DNA-Antikörper bei. Hinweisend auf einen systemischen Lupus erythematodes kann ferner die Existenz von Anti-CardiolipinAntikörpern sein (Grünig, Barner et al. 2010). Bei dem Vorliegen einer chronischthromboembolischen pulmonalen Hypertonie empfehlen sich die Durchführung einer Gerinnungsdiagnostik, sowie der Ausschluss eines Antiphospholipid-Syndroms. Zum Screening sowie zur initialen Risikoabwägung als auch zur Verlaufskontrolle eignet sich die Bestimmung von BNP und NT-proBNP. Patienten mit einem Serum NT-proBNP-Wert $\geq 1400 \mathrm{pg} / \mathrm{ml}$ haben eine schlechtere Langzeitprognose als 
Patienten, welche niedrigere Werte aufweisen (Fijalkowska, Kurzyna et al. 2006). Hinweisend auf eine myokardiale Schädigung und prognostisch bedeutend bei einer akuten Lungenembolie, einem akuten Koronarsyndrom sowie bei einer chronischen PAH oder CTEPH sind erhöhte Werte der Plasmatroponine T sowie I (Eggers, Nygren et al.; Heresi, Tang et al.; Torbicki, Kurzyna et al. 2003). Bei Patienten mit einer pulmonalen Hypertonie können erhöhte Endothelinspiegel nachgewiesen werden (Wilkens, Bauer et al. 2003). Endothelin besitzt neben einer starken vasokonstriktorische Wirkung auch proinflammatorische Eigenschaften.

\subsubsection{Echokardiographie}

Bei allen Patienten mit dem Verdacht einer pulmonalen Hypertonie sollte ein echokardiographisches Screening durchgeführt werden. Dies ist eine gut geeignete nicht-invasive Methode zur Verlaufskontrolle sowie Therapieeinschätzung bei PH-Patienten. Die Untersuchung hilft bei der Differentialdiagnose, ob eine linksventrikuläre Dysfunktion, Klappenfehler oder auch kongenitale Vitien (Shunts) ursächlich für die pulmonale Hypertonie sind. Zudem werden typische Befundkonstellationen einer manifesten $\mathrm{PH}$ aufgezeigt, welche für die prognostische Einschätzung des Patienten von Bedeutung sind. $\mathrm{Zu}$ diesen zählen ein dilatiertes rechtes Atrium, ein vergrößerter rechter Ventrikel, ein abgeflachtes Septum mit paradoxem Bewegungsmuster eine dilatierte Vena cava inferior, sowie der Nachweis eines Perikardergusses (Raymond, Hinderliter et al. 2002). Eine Abschätzung des systolischen pulmonalarteriellen Druckes ist mit Hilfe der dopplersonographischen Messung der Geschwindigkeit des Trikuspidalreflux-Jets möglich, jedoch teilweise fehlerbehaftet. Der Normbereich liegt unter Ruhebedingungen bei unter 2,8 m/s, bei einer leichten Form der $\mathrm{PH}$ bei 2,8-3,4 m/s und bei mittelgradiger $\mathrm{PH}$ bei über 3,4 m/s (Masuyama, Kodama et al. 1986). Zu diesem Druck erfolgt die Addition des geschätzten rechtsatrialen Druckes beziehungsweise 5-15 $\mathrm{mmHg}$ abhängig vom momentanen Kompensationszustand des Patienten. Des Weiteren erfolgt die Bestimmung des Tei-Indexes (myokardialer Performance-Index, MPI). Dieser setzt sich zusammen aus der isovolumetrischen Relaxationszeit sowie der isovolumetrischen Kontraktionszeit dividiert durch die Ejektionszeit (Tei, Ling et al. 1995) (Normwert < 0,3 für den rechten Ventrikel). Prognostisch ungünstig ist ein Tei-Index von > 0,83 (Yeo, Dujardin et al. 1998). Die TAPSE (tricuspid annular plane systolic excursion) hilft neben dem Tei-Index bei der Beurteilung einer 
globalen rechtsventrikulären Funktionsstörung. Normwerte sind im Bereich von $21 \pm 2,7 \mathrm{~mm} \mathrm{zu}$ sehen, pathologische Werte bei unter $20 \mathrm{~mm}$ (Forfia, Fisher et al. 2006).

Bestätigt sich der Verdacht eines Lungenhochdrucks durch die Echokardiographie, ist die weitere invasive Abklärung mittels Rechtsherzkatheter beziehungsweise Linksherzkatheter dringend anzuraten, da eine definitive Diagnose nur mittels dieser Diagnostiken gewonnen werden kann (Grünig and Ley 2008).

\subsubsection{Ventilations-Perfusions-Szintigraphie}

Bei jedem Patienten mit einer pulmonalen Hypertonie unklarer Ätiologie sollte eine Ventilations-Perfusionsszintigraphie (V/Q-Szintigraphie) durchgeführt werden. Dies ermöglicht den Ausschluss einer chronisch-thromboembolischen pulmonalen Hypertonie beziehungsweise hilft bei deren Diagnosestellung. Die V/Q-Szintigraphie ist sensitiver als die Computertomographie und ist daher die Methode der Wahl zum Screening einer potentiell kurativ behandelbaren CTEPH (Tunariu, Gibbs et al. 2007). Es zeigen sich bei PAH-Patienten lediglich kleine Inhomogenitäten der Perfusion, während bei CTEPH größere Perfusionsdefekte nachweisbar sind. Bei der chronisch-thromboembolischen pulmonalen Hypertonie liefert die V/Q-Szintigraphie segmentale Perfusionsausfälle mit einem Missmatch zur Ventilation (Wilkens 2010).

\subsubsection{Computertomographie/ Pulmonalisangiographie}

Die Durchführung einer kontrastmittelgestützten Computertomographie in Spiraltechnik gibt wichtige Hinweise zur Beurteilung der Operabilität einer chronisch-thromboembolischen pulmonalen Hypertonie (Reichelt, Hoeper et al. 2009). Zusätzlich ist eine Beurteilung des Lungenparenchyms möglich. Bei erfahrenen Untersuchern ist zur Indikationsstellung einer potentiellen Endarteriektomie die Pulmonalisangiographie ein sicheres Verfahren und liefert zudem gegenüber der Computertomographie zusätzliche Hinweise auf die Art des obstruierenden Materials in der Lungenstrombahn (Fedullo, Auger et al. 2001).

\subsubsection{Magnetresonanztomographie}

Die Magnetresonanztomographie (MRT) ist eine sehr gute Möglichkeit zur nicht-invasiven Beurteilung der rechtsventrikulären Morphologie, Größe und Funktion. Des Weiteren wird die Bestimmung des Schlagvolumens, des pulmonalen Blutflusses sowie des Herzzeitvolumens und der Dehnbarkeit der Pulmonalarterien 
ermöglicht (Marcus, Gan et al. 2008). Prognostisch ungünstig sind ein erniedrigtes linksventrikuläres sowie ein erhöhtes rechtsventrikuläres enddiastolisches Volumen als auch ein reduziertes Schlagvolumen (Torbicki 2007). Neuere MRT-Verfahren ermöglichen zudem die Beurteilung der Lungenperfusion. Zurzeit erfolgt in Studien die Begutachtung des Stellenwertes dieser Untersuchung zur Verlaufskontrolle bei Patienten mit einer pulmonalen Hypertonie (Grünig, Barner et al.).

\subsubsection{Herzkatheteruntersuchung}

Die Rechtsherzkatheteruntersuchung gehört zur Standarddiagnostik der pulmonalen Hypertonie. Mittels Swan Ganz-Katheter wird die genaue Beurteilung der hämodynamischen Parameter des kleinen Kreislaufs ermöglicht. Es erfolgt die Ermittlung des pulmonalkapillären Verschlussdruckes (PAWP) sowie die Bestimmung des pulmonalarteriellen Druckes (systolisch, diastolisch, Mitteldruck). Dies ermöglicht eine Differenzierung zwischen prä- und postkapillären Ursachen einer pulmonalen Hypertonie. Weitere zu erfassende Parameter sind der rechtsatriale Druck (RAP), der rechtsventrikuläre Druck (RVP) und der transpulmonale Gradient (TPG). Der TPG ergibt sich aus der Differenz zwischen dem pulmonal arteriellen Mitteldruck und dem pulmonalkapillären Verschlussdruck (TPG=PAPm-PCWPm, Normwert $\leq 12 \mathrm{mmHg}$ ) (Opitz, Blindt et al.). Des Weiteren erfolgt mittels Thermodilutionsmethode oder nach dem Fick'schen Prinzip die Ermittlung des Herzzeitvolumens (HZV). Aus den genannten Parametern kann schließlich der pulmonal vaskuläre Widerstand errechnet werden (PVR). Außerdem wird die Sauerstoffsättigung systemisch arteriell, pulmonal arteriell sowie in der Vena cava superior bestimmt. Dieses erfolgt zum Ausschluss eventuell vorliegender Shuntvitien. Definitionsgemäß handelt es sich um eine pulmonale Hypertonie bei der Erhöhung des pulmonalarteriellen Mitteldruckes auf $\geq 25 \mathrm{mmHg}$ gemessen unter Ruhebedingungen. Das Herzzeitvolumen ist entweder normal oder leicht vermindert. Der PAWP ist bei Patienten mit einer präkapillären pulmonalen Hypertonie normwertig, also $\leq 15 \mathrm{mmHg}$. Eine Erhöhung des PVR auf $>240 \mathrm{dyn} / \mathrm{sec} / \mathrm{cm}^{-5}$ resultiert aus dem Anstieg den pulmonalarteriellen Druckes und des Herzzeitvolumen (Badesch, Champion et al. 2009; Galie, Hoeper et al. 2009). Eine Erhöhung des PAWP ist meist Resultat einer Linksherzerkrankung mit pulmonaler Stauung. Eine Übersicht der hämodynamischen Definition der pulmonalen Hypertonie ist in Tabelle $6 \mathrm{zu}$ sehen. Konnte mittels des Rechtsherzkatheters der 
Nachweis einer pulmonalen Hypertonie erbracht werden, erfolgt bei Patienten mit PAH in der gleichen Untersuchung die Durchführung eines Vasoreagibilitätstests. Dieser dient der Identifizierung der Patienten, welche von einer hochdosierten Therapie mittels Kalziumantagonisten profitieren können („Responder“). Die Durchführung der Testung sollte nur mit kurzwirksamen pulmonalen Vasodilatatoren

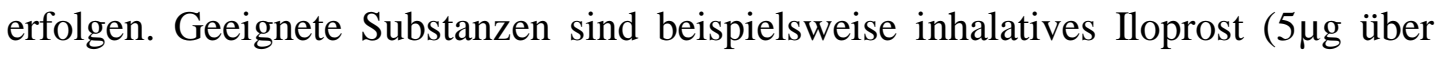
5-10 min) oder auch inhalatives NO (10-20 ppm über 5-10 min) (Badesch, Champion et al. 2009; Jing, Jiang et al. 2009). Ein positives Ergebnis ist durch den Abfall des pulmonal arteriellen Mitteldrucks (PAPm) um mindestens $10 \mathrm{mmHg}$ auf unter 40mmHg, mit ansteigendem beziehungsweise unverändertem Herzzeitvolumen, definiert. Nur ein kleiner Teil der IPAH beziehungsweise HPAH-Patienten $(<10 \%)$ profitiert von der Therapie mittels Kalziumantagonisten (Sitbon, Humbert et al. 2005), bei allen anderen PAH Formen gelang kein Nachweis eines therapeutischer Effekts.

\begin{tabular}{|l|c|}
\hline \multicolumn{1}{|c|}{ Definition } & Kennzeichen \\
\hline Pulmonale Hypertonie & PAPm $\geq 25 \mathrm{mmHg}$ \\
\hline Präkapilläre PH & PAPm $\geq 25 \mathrm{mmHg}$ \\
& PAWP $\leq 15 \mathrm{mmHg}$ \\
& $\mathrm{HZV} \downarrow$ oder normal \\
\hline Postkapilläre PH & PAPm $\geq 25 \mathrm{mmHg}$ \\
& PAWP $>15 \mathrm{mmHg}$ \\
passiv & $\mathrm{HZV} \downarrow$ oder normal \\
reaktiv (out of proportion) & $\mathrm{TPG} \leq 12 \mathrm{mmHg}$ \\
\hline TPG- transpulmonaler Gradient (PAPm-PAWP) & $\mathrm{TPG}>12 \mathrm{mmHg}$ \\
PAWP-mittlerer pulmonal-kapillärer Verschlussdruck \\
\hline
\end{tabular}

Tabelle 6: Hämodynamik der PH (Opitz, Blindt et al.)

\subsection{Therapieoptionen der PAH}

Eine Heilung der PAH ist auch im Zuge der Entwicklung neuer spezifischer Medikamente immer noch nicht möglich. Jedoch haben sich in den letzen Jahren die Therapiemöglichkeiten für Patienten mit pulmonaler Hypertonie zunehmend verbessert. Nach Durchführung einer gezielten Diagnostik und ätiologischer Klassifikation kann die Einleitung einer spezifischen Therapie erfolgen. Dies gilt insbesondere für symptomatischen Patienten, also Patienten welche sich in der WHO functional class III oder IV befinden (Rosenkranz 2007). Für einzelne Medikamente konnte die Therapie-Indikation auch auf weniger schwere Funktionseinschränkungen 
erweitert werden (Galie, Rubin et al. 2008). Bisher besteht bei den spezifischen Therapeutika lediglich eine Zulassung in der Gruppe 1 (pulmonal arterielle Hypertonie), während die anderen Formen der PH noch nicht eingeschlossen wurden. Wesentliche Ziele der Therapie sind zum einen die Verminderung des pulmonal-vaskulären Widerstandes und zum anderen eine Reduktion der pulmonal arteriellen Hypertonie sowie ein Rückgang der rechtsventrikulären Dilatation beziehungsweise Hypertrophie. Ein weiterer wichtiger therapeutisch anzugehender Punkt ist die Verbesserung der körperlichen Belastbarkeit und damit der Lebensqualität. Dies wird am ehesten durch eine Erhöhung des Herzzeitvolumens und die damit verbundene verbesserte Sauerstoffzufuhr in den peripheren Organen und im Gewebe ermöglicht. Zu den allgemeinen und supportiven Maßnahmen in der Behandlung zählen die Gabe von Sauerstoff, Diuretika, Digitalis-Präparaten (Galie, Hoeper et al. 2009). Nach Expertenmeinung wird auch für PAH-Formen, welche nicht als CTEPH eingestuft werden, eine orale Antikoagulation als indiziert erachtet. Für die gezielte medikamentöse Therapie der PAH stehen mittlerweile die folgenden drei Substanzklassen zur Verfügung: Phosphodiesterase 5-Inhibitoren (PDE 5Inhibitoren), Endothelin-Rezeptor-Antagonisten (ERA) sowie Prostazyklin-Analoga. Je nach Schwere des Krankheitsverlaufes beziehungsweise bei unzureichendem Ansprechen auf eine spezifische Monotherapie kann eine Kombinationstherapie erfolgen (Behr, Borst et al. 2005) (Hoeper, Markevych et al. 2005). Als letzte Behandlungsoption beziehungsweise bei fehlendem Ansprechen auf eine Kombinationstherapie besteht die Möglichkeit einer Ballonatrioseptostomie oder Lungentransplantation (Ghofrani, Distler et al.). Bei Patienten mit einer chronisch thromboembolischen pulmonalen Hypertonie ist primär das chirurgische Verfahren der pulmonalen Endarteriektomie (PEA) anzustreben, die Operabilität sollte bei jedem Patienten durch einen PEA-Chirurgen beurteilt werden.

\subsubsection{Kalzium-Antagonisten}

Die Empfehlung zur Verwendung von Kalzium-Antagonisten wird nur für PAH-Patienten gegeben, welche die Responder-Kriterien bei der invasiven Diagnostik mittels Rechtsherzkatheter erfüllen (Sitbon, Humbert et al. 2005). Sollten die Patienten unter der Behandlung mit Kalzium-Antagonisten keine Besserung der Hämodynamik aufweisen oder eine Verschlechterung des funktionellen 
NYHA-Stadiums auftreten, wird eine Änderung der Therapiestrategie mit anderen gezielten PAH-Medikamenten befürwortet (Barst, Gibbs et al. 2009).

\subsubsection{Phosphodiesterase 5- Inhibitoren}

Aufgabe der Phosphodiesterase 5-Inhibitoren (PDE 5-Inhibitoren) ist die Verstärkung der vasodilatativen Wirkung von endogenem Stickoxid (NO). Bedingt durch die Hemmung der Phosphodiesterase 5 kommt es zu einem Anstieg des intrazellulären zyklischem Guanosinmonophosphat (cGMP) durch dessen verminderten Abbau (Mehta 2003). In randomisierten, placebokontrollierten Studien, wie der PHIRST-Studie (Tadalafil) und der SUPER-Studie (Sildenafil), konnte eine Verbesserung der Hämodynamik sowie der Leistungsfähigkeit nachgewiesen werden (Galie, Ghofrani et al. 2005; Galie, Brundage et al. 2009).

\subsubsection{Prostazyklin-Analoga}

Die Substitution der Prostazykline verursacht durch die Adenylatzyklase- abhängige Freisetzung von zyklischem Adenosinmonophosphat (cAMP) eine Vasodilatation. Sie führt zu einer Hemmung der Proliferation der glatten Gefäßmuskelzellen sowie der Thrombozytenaggregation (Rosenkranz 2007). Aufgrund der Instabilität von Prostazyklin erfolgte zur medikamentösen Behandlung die Entwicklung verschiedener Prostazyklin-Analoga. 1995 erfolgte die Zulassung des intravenös zu verabreichende Medikaments Epoprostenol zur Behandlung der pulmonal arteriellen Hypertonie (Barst, Gibbs et al. 2009). Hierzu ist jedoch die intravenöse Applikation mittels transportabler Pumpensysteme erforderlich. Für das inhalative Prostanoid Iloprost konnte in der AIR-Studie, welche PAH-Patienten sowie Patienten mit einer CTEPH einschloss, eine Verbesserung der Hämodynamik und der körperlichen Leistungsfähigkeit nachgewiesen werden. Es kam zu einer Verbesserung der Atemnot sowie des Schweregrades der Erkrankung (Olschewski, Simonneau et al. 2002).

\subsubsection{Endothelin-Rezeptor-Antagonisten}

Die Endothelin-Rezeptoren befinden sich im Endothel sowie in den glatten Gefäßmuskelzellen. Durch deren Inhibition kommt es zu einer Vasodilatation sowie $\mathrm{zu}$ einem antiproliferativen Effekt. Patienten mit pulmonaler Hypertonie weisen erhöhte Endothelinspiegel auf. Endothelin besitzt neben proinflammatorischen Eigenschaften auch eine starke vasokonstriktorische Wirkung (Barton and 
Yanagisawa 2008). Die zurzeit zugelassenen Substanzen der Endothelin-RezeptorAntagonisten (ERA) sind Bosentan sowie Ambrisentan. Bosentan bewirkt eine duale Blockade des Endothelin A- und Endothelin-B-Rezeptors. Randomisierte Studien zeigten unter Therapie eine Steigerung der körperlichen Leistungsfähigkeit, eine Besserung des klinischen Schweregrades der Erkrankung sowie eine Verbesserung der hämodynamischen Parameter. Des Weiteren konnte die Zeit bis zur klinischen Verschlechterung hinausgezögert werden (Galie, Rubin et al. 2008) (Barst, Gibbs et al. 2009). Ambrisentan blockiert im Gegensatz zu Bosentan selektiv den $\mathrm{ET}_{\mathrm{A}^{-}}$ Rezeptor. Ein Vorteil gegenüber der dualen Blockade ist bisher nicht nachgewiesen. Unter der Therapie mit Endothelin-Rezeptor-Antagonisten kann es aufgrund der potentiellen Hepatotoxizität zu einem Anstieg der Transaminasen kommen (Barst, Gibbs et al. 2009). Daher sind vierwöchige Laborkontrollen für den Zeitraum der Behandlung indiziert.

\subsection{Historisches zur Impedanzmessung}

Die Ursprünge der Bestimmung des Herzzeitvolumens mit Hilfe der Impedanzkardiographie sind in den 30er Jahren zu finden. Atzler und Lehmann entwickelten eine Methode zur Registrierung von Kapazitätsänderungen, welche darauf beruhten, dass Thorax und Herz der zu untersuchenden Person zwischen die Platten eines Kondensators gebracht wurden und die Volumenschwankungen pro Herzschlag ermittelt wurden (Atzler 1932). Ein grundlegender Faktor dieser Methode ist die Dielektrizitätskonstante sowie das Volumen des eingebrachten Körpers. Die Dielektrizitätskonstante von Wasser beziehungsweise Blut beträgt etwa 81, die der Luft entspricht 1. Da sich das Herz innerhalb eines Herzzyklus im luftgefüllten Thorax vergrößert und verkleinert, nahmen Atzler und Lehmann die Differenzen der Dielektrizitätskonstanten zur Registrierung von Volumenschwankungen. Dies war die Geburtsstunde des ersten Dielektrographen. Die endgültige Anwendung dieser Methode erfolgte jedoch erst in den 60er Jahren durch Kubicek zur Gewinnung von Informationen über das Verhalten des HerzKreislaufsystems bei Astronauten im Zustand der Schwerelosigkeit (Kubicek, Karnegis et al. 1966). Die Akzeptanz dieser Methode war aufgrund der Umständlichkeit der Anwendung, mit um den Thorax gewickelten Aluminiumstreifen und der zeitintensiven Berechnung der Daten relativ gering. Des Weiteren gingen die Meinungen hinsichtlich der Präzision der transthorakalen 
Impedanzmessung im Vergleich zur Thermodilution auseinander (Naggar, Dobnik et al. 1975; Keim, Wallace et al. 1976). Die von Kubicek entwickelte Formel zur Berechnung des Schlagvolumens mittels Impedanzkardiographie wurde mehrfach modifiziert, unter anderem von Sramek (Sramek 1981) sowie Bernstein (Bernstein 1986).

Der NCCOM3-R7S ist ein System, mit welchem nicht-invasiv, basierend auf Impedanzänderungen im Thorax zwischen Systole und Diastole, die hämodynamischen Parameter entweder kontinuierlich (von Herzschlag zu Herzschlag) oder gemittelt bestimmt werden können. Die entstehenden Impedanzänderungen werden mittels normaler EKG-Elektroden erfasst (siehe Abbildung 3). In der vorliegenden Untersuchung erfolgte eine nicht-invasive Beurteilung der Hämodynamik auf Basis dieser Technik bei Patienten mit pulmonaler Hypertonie und gesunden Vergleichspersonen unter Belastungsbedingungen.

\subsection{Kardiale Bioimpedanzmessung}

Bei der Impedanz (Z [Ohm]), auch Wechselstromwiderstand, handelt es sich um den Quotienten aus elektrischer Spannung und Stromstärke. Sie repräsentiert den frequenzabhängigen Widerstand eines Leiters. Für Wechselströme von $20-100 \mathrm{kHz}$ weist die Impedanz des Thorax pulssynchrone und atemabhängige Änderungen auf. Diese Gegebenheit nutzten bereits in den 30er Jahren Atzler und Lehmann zur Ermittlung des Herzzeitvolumens mit ihrem Dielektrographen (Atzler 1932). Der Anteil elektrisch gut leitender extrazellulärer Flüssigkeit ermöglicht die Bestimmung der Impedanz. Eine Differenzierung zwischen thorakaler und Ganzkörperimpedanz wird durch die Positionierung der Ableitelektroden erlaubt. In den 60er Jahren entwickelten Kubicek et al. eine Methode zur Bestimmung des Herzzeitvolumens (HZV) mittels der thorakalen Bioimpedanzmessung. Grundlage der Berechnungen ist die Überlegung, dass der Thorax als ein mit Flüssigkeit gefüllter Zylinder betrachtet wird (van der Meer, Woltjer et al. 1996). Das Schlagvolumen ergibt sich aus der Änderung der Flüssigkeitsmenge. In den 80er Jahren wurde die Formel zur Berechnung des HZV von Sramek dahingehend modifiziert, dass er den Thorax nicht als Zylinder, sondern als einen Kegelstumpf definierte und einen Korrekturfaktor für das Körpergewicht einsetzte (van der Meer, Woltjer et al. 1996). 
Die Änderungen der Impedanz sind neben dem erhöhten aortalen Volumen zum Zeitpunkt des Auswurfes auch von weiteren Faktoren abhängig, wie beispielsweise dem Luftgehalt der Lunge. Darüber hinaus kommt es zu einer Ausrichtung der Erythrozytenfläche parallel zur Flussrichtung, hierdurch ergibt sich bei der Messung in Flussrichtung eine höhere Leitfähigkeit des Blutes (Hoeft 2008).

Bei dem in dieser Studie genutzten nichtinvasiven hämodynamischen Monitoring der kardialen Bioimpedanz ist zur Registrierung der Impedanzänderungen eine modifizierte Elektrodenanordnung erforderlich. Es werden 4 Elektrodenpaare (AgCl-Elektroden) benötigt. Zur genauen und reproduzierbaren Messung sollten die Ableitknöpfe von zwei Elektroden $5 \mathrm{~cm}$ voneinander entfernt befestigt werden. Die Anordnung der Elektroden ist wie folgt: jeweils ein Elektrodenpaar auf der rechten und linken Halsseite (Frontalebene) sowie an der rechten und linken lateralen Thoraxapertur in Höhe des Xyphoids in der mittleren Axillarlinie (Castor 1989). Ein elektrischer Wechselstrom von wenigen Milliampere wird über die äußeren Elektroden durch den Thorax geleitet (siehe Abbildung 2), der Strom sucht sich den Weg des geringsten Widerstandes, in diesem Fall die Aorta. Die inneren Elektroden erfassen stromlos den Spannungsabfall und somit die thorakale Impedanz. Es erfolgte eine EKG-Ableitung mit zwei Brustwandelektroden sowie die Eingabe von Geschlecht, Körpergewicht und Körpergröße der zu untersuchenden Person. Der NCCOM3-R7S (BoMed) ist ein System welches auf dieser Methode basiert. Mit Unterstützung von zwei Mikroprozessoren werden die unter Punkt 3.3 aufgeführten physiologischen und hämodynamischen Parameter berechnet und basierend auf der Körperoberfläche (KOF) oder als Absolutwert im Display des NCCOM3-R7S angezeigt. 


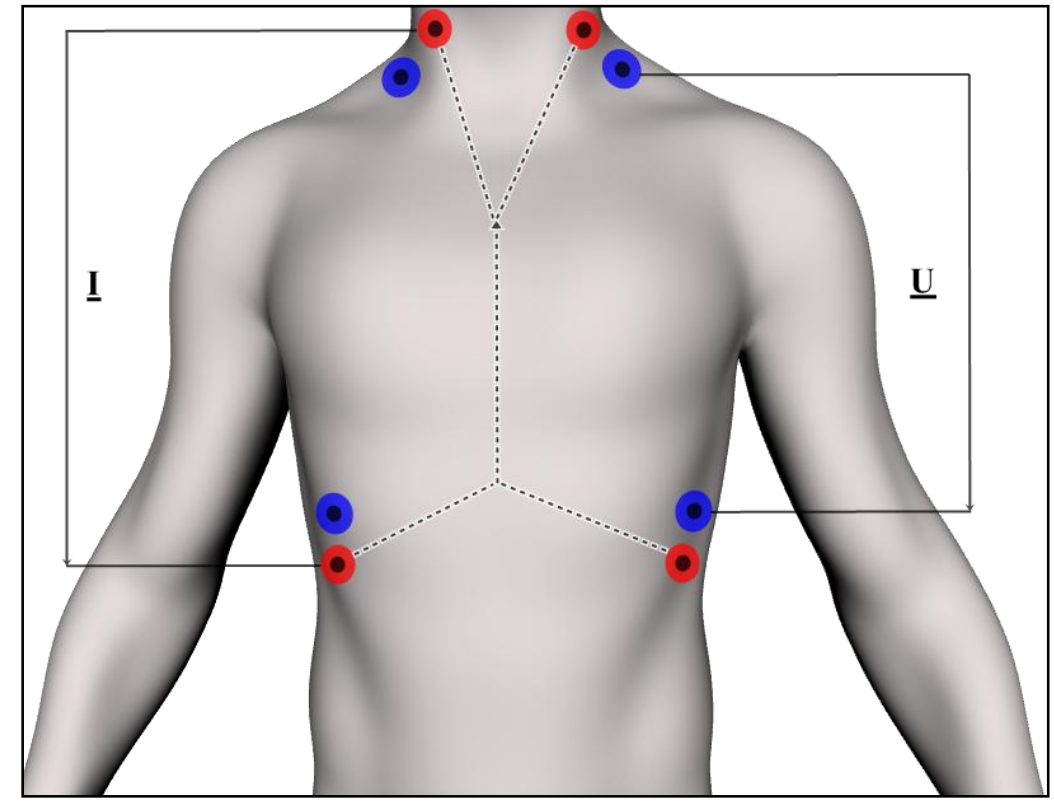

Abbildung 2: Elektrodenanordnung zum Monitoring der kardialen Bioimpedanz

\subsection{Ziel der Arbeit}

Etwa $80 \%$ aller Patienten mit einer pulmonalen Hypertonie befinden sich zum Zeitpunkt der Diagnosestellung in der WHO functional class I-III (Humbert, Sitbon et al. 2006) und sind somit unter Ruhebedingungen weitestgehend beschwerdefrei. Die eigentlichen, limitierenden Beschwerden, wie Dyspnoe und thorakales Druckgefühl, treten vor allem unter körperlicher Anstrengung auf. Die Hämodynamik unter Ruhebedingungen wurde bisher ausführlich untersucht. Dagegen liegen so gut wie keine Daten hinsichtlich des hämodynamischen Verhaltens unter körperlicher Belastung vor. Die Diagnostik mit Hilfe der kardialen Bioimpedanz könnte eine gute Methode zur nicht-invasiven Beurteilung des Verhaltens der hämodynamischen Parameter unter Belastungsbedingungen sein.

Besonderes Augenmerk sollte auf zwei Gegebenheiten geworfen werden. Zum Einen ist das Leitsymptom der Patienten mit einer pulmonalen Hypertonie die Belastungsdyspnoe. Diesbezügliche Diagnostik erfolgte jedoch bisher vornehmlich unter Ruhebedingungen. Zum Anderen liegen nur unzureichende Daten in Bezug auf die Hämodynamik (wie beispielsweise der mPAP) unter Belastung vor.

Ziel der vorliegenden Arbeit ist es zu untersuchen, ob die kardiale Bioimpedanz zur Beurteilung der linksventrikulären Parameter unter Belastung geeignet ist und ob die spezifischen Parameter dieser Methodik für die Beurteilung der Hämodynamik unter Belastung von zusätzlichem Wert sein können. 


\section{Material und Methodik}

\subsection{Patientenkollektiv}

Alle Patienten mit einer pulmonalen Hypertonie, bei denen im Rahmen der klinischen Diagnostik eine Spiroergometrie geplant war, wurden gefragt, ob sie während dieser Untersuchung gleichzeitig an der Bioimpedanz-Studie teilnehmen wollten. Die Kontrollpersonen wurden unter den Mitarbeitern der Inneren Medizin V rekrutiert. Anschließend erfolgte nach Zustimmung der Patienten beziehungsweise der gesunden Probanden zunächst die Erhebung der persönlichen Daten (wie Name, Geburtsdatum, Gewicht, Größe, Geschlecht, aktuelle Medikation).

Die Erhebung der Daten erfolgte über einen Zeitraum von 13 Monaten. Die Kontrollgruppe schloss 19 gesunde Probanden ein. In der Gruppe der Patienten mit pulmonaler Hypertonie befanden sich 24 Patienten, welche sich in der WHO-Klasse II oder III befanden. Das Patientenkollektiv umfasste sowohl Patienten mit einer pulmonalarteriellen Hypertonie als auch Patienten mit einer chronisch thromboembolischen pulmonalen Hypertonie (siehe Tabelle 7).

\section{Basisparameter}

\begin{tabular}{|l|c|c|c|c|}
\hline & $\begin{array}{c}\text { Kontrolle } \\
\text { MW } \pm \text { SD }\end{array}$ & $\begin{array}{c}\text { Patienten } \\
\text { MW } \pm \text { SD }\end{array}$ & $\begin{array}{c}\text { PAH } \\
\text { MW } \pm \text { SD }\end{array}$ & $\begin{array}{c}\text { CTEPH } \\
\text { MW } \pm \text { SD }\end{array}$ \\
\hline Anzahl [n] & 19 & 24 & 13 & 11 \\
\hline Alter [Jahre] & $47,6 \pm 14,7$ & $57,1 \pm 17,4$ & $53,2 \pm 18$ & $61,7 \pm 16,3$ \\
\hline Geschlecht [m/ w] & $11 / 8$ & $5 / 19$ & $1 / 12$ & $4 / 7$ \\
\hline WHO- FC & 0 & II - III & II - III & II - III \\
\hline
\end{tabular}

Die hämodynamischen Parameter der Patienten sind in Tabelle 8 zusammengefasst. Der pulmonal-vaskuläre Widerstand des Patientenkollektives lag im Mittel bei 940 dyn* $\mathrm{sec}^{*} \mathrm{~cm}^{-5}$. Die Erhebung der Daten erfolgte invasiv mittels Rechtsherzkatheter entweder zum Zeitpunkt der Diagnosestellung oder im Zuge des Krankheitsverlaufs. 


\section{Hämodynamik}

\begin{tabular}{|c|c|c|c|}
\hline & $\begin{array}{l}\text { Patienten } \\
M W \pm S D\end{array}$ & $\begin{array}{c}\text { PAH } \\
\mathrm{MW} \pm \mathrm{SD}\end{array}$ & $\begin{array}{c}\text { CTEPH } \\
\mathrm{MW} \pm \mathrm{SD}\end{array}$ \\
\hline $\mathbf{P A P}_{\text {sys }}[\mathrm{mmHg}]$ & $83,4 \pm 24,9$ & $82 \pm 22,6$ & $84,9 \pm 28,5$ \\
\hline $\mathbf{P A P}_{\text {dia }}[\mathrm{mmHg}]$ & $35,4 \pm 15,4$ & $38,9 \pm 16,8$ & $31,6 \pm 13,6$ \\
\hline $\mathbf{P A P}_{\mathrm{m}}[\mathrm{mmHg}]$ & $49,4 \pm 12,8$ & $51,2 \pm 13,0$ & $46,5 \pm 12,1$ \\
\hline PAWP[mmHg] & $11 \pm 3,0$ & $10,7 \pm 2,1$ & $11,4 \pm 3,9$ \\
\hline HZV $\quad[\mathrm{l} / \mathrm{min}]$ & $3,8 \pm 1,1$ & $4,0 \pm 1,1$ & $3,5 \pm 0,9$ \\
\hline PVR $\left[\mathrm{dyn}^{*} \sec ^{*} \mathrm{~cm}^{-5}\right]$ & $948,0 \pm 454,8$ & $1024,5 \pm 591,6$ & $880,0 \pm 310,5$ \\
\hline
\end{tabular}

Tabelle 8: Hämodynamische Eigenschaften des Patientenkollektivs

Die in unsere Datenerhebung eingeschlossenen Patienten wiesen keine Reizüberleitungsstörungen oder kardiale Arrhythmien auf. Sie erhielten darüber hinaus keine negativ chronotrope oder inotrope Medikation. In der Vorgeschichte des Patientenkollektives lagen in Bezug auf die Atemwege keine signifikanten Erkrankungen vor. Des Weiteren erfolgte der Ausschluss derjenigen Patienten, welche an einer koronaren Herzkrankheit oder Klappenvitien litten. Im Rahmen der Datenermittlung konnte unter Ruhebedingungen eine erhaltene systolische linksventrikuläre Funktion echokardiographisch nachgewiesen werden.

\subsection{Versuchsanordnung}

Alle Teilnehmer der Untersuchung waren über den Versuchsablauf aufgeklärt.

Nach der Zustimmung für die geplante Untersuchung wurden alle fahrradergometrischen Einstellungen vorgenommen und die EKG-Elektroden angelegt. Nach dem Reinigen der Haut an den lateralen Halsseiten sowie an der unteren Thoraxapertur erfolgte die Anlage der vier Elektrodenpaare im Abstand von jeweils fünf Zentimetern zur Ableitung der Parameter der kardialen Bioimpedanz. Des Weiteren wurden zwei zusätzliche Elektroden, welche eine EKG-getriggerte Darstellung der $\mathrm{zu}$ erhebenden Parameter erlauben, angebracht. Als Nächstes erfolgten unter Ruhebedingungen die Ermittlung des Blutdrucks sowie des RuheEKGs.

Mit Hilfe der kardialen Bioimpedanz konnten folgende Parameter nicht-invasiv erhoben werden. Als Basisparameter wurden die Herzfrequenz, das Schlagvolumen, das Herzminutenvolumen, die linksventrikuläre Ejektionsfraktion sowie das linksventrikuläre enddiastolische Volumen bestimmt. Des Weiteren erfolgte die Berechnung des Beschleunigungsindexes, des Kontraktilitätsindexes sowie des Peak Flows. Der Verlauf des Auswurfes zeigte sich in der ventrikulären Ejektionszeit 
(VET) sowie im Auswurfverhältnis, welches sich aus der VET und dem RR-Intervall zusammensetzt (siehe Tabelle 9).

\section{Basisparameter}

\begin{tabular}{|l|r}
\hline Herzfrequenz (HF) & {$[1 / \mathrm{min}]$} \\
\hline Schlagvolumen (SV) & {$[\mathrm{ml}]$} \\
\hline Herzminutenvolumen (HMV) & {$[1 / \mathrm{min}]$} \\
\hline Herzindex (CI) & {$\left[1 / \mathrm{min} / \mathrm{m}^{2}\right]$} \\
\hline Ejektionsfraktion (EF) & {$[\%]$} \\
\hline Enddiastolisches Volumen (EDV) & {$[\mathrm{ml}]$} \\
\hline
\end{tabular}

\section{Flussparameter}

Peak Flow (PF) $[\mathrm{ml} / \mathrm{sec}]$

Kontraktilitätsindex (IC)

Beschleunigungsindex (ACI) $[1 / \mathrm{sec}]$

Zeitparameter

Ventrikuläre Ejektionszeit (VET)

Auswurfverhältnis (ER) $=$ VET/RR-Intervall

$[\mathrm{msec}]$

Tabelle 9: Parameter der kardialen Bioimpedanz

Während der Belastungsuntersuchung, welche mit Hilfe einer konventionellen Spiroergometrie durchgeführt wurde, erfolgte an sechs vorher festgelegten Messpunkten die Erhebung der eingangs erwähnten Parameter. Die erste Datenaufnahme fand unter Ruhebedingungen (BL) statt. Weitere Zeitpunkte waren während des Leertretens (UP) sowie in der frühen Belastungsphase (EE). Die Festlegung der frühen Belastungsphase erfolgte, auf Grundlage eines Blutdruckanstieges beziehungsweise Pulsanstieges von 10-20\% in Relation zur Phase des Leertretens. Weitere Datenaufzeichnungen fanden im Bereich der anaeroben Schwelle (AT) sowie unter submaximaler Belastung (SM) statt. Die Festlegung der anaeroben Schwelle erfolgte nach Erreichen eines respiratorischen Quotienten von 1. Die letzten Messwerte wurden in der Erholungsphase (RC) ermittelt, drei Minuten nach Belastungsende.

\subsection{Parameter der kardialen Bioimpedanz}

\subsubsection{Peak Flow Index}

$$
P F=V E P T \times I C \times \text { const }\left[\frac{m l}{s e c}\right]
$$

Formel 1: Berechnung des Peak Flow 
Der Peak Flow (PF) repräsentiert den maximalen links-ventrikulären Blutfluss während der Austreibungsphase. Des Weiteren wird er als ein Parameter der Kontraktilität angesehen und ist als solcher abhängig vom Füllungsvolumen des linken Ventrikels.

$$
P F I=\frac{\text { PeakFlow }(P F)}{\text { Körperoberfläche }(K O F)}\left[\mathrm{ml} / \mathrm{sec} / \mathrm{m}^{2}\right]
$$

Formel 2: Berechnung des Peak Flow Indexes

Normal: $\quad 170-370 \mathrm{ml} / \mathrm{sec} / \mathrm{m}^{2}(1992)$

Der Peak Flow Index (PFI) ist volumenabhängig. Dies bedeutet, dass er bei hypovolämischen Patienten trotz der Möglichkeit eines normalen Inotropiestatus niedriger ausfallen wird. Gleiches gilt für den Kontraktilitätsindex.

\subsubsection{Ejektions-Fraktion}

$$
E F=\frac{\text { Schlagvolumen }(S V)}{\text { enddiastolisches Volumen }(E D V)}[\%]
$$

Formel 3: Berechnung der Ejektionsfraktion

$$
\text { linksventrikuläre } E F=0,84-0,64 \times\left(\frac{P E P}{L V E T}\right)
$$

Formel 4: Algorithmus der linksventrikulären Ejektionsfraktion von L. Capan (Capan 1987)

PEP Praeejektionsperiode

LVET linksventrikuläre Austreibungszeit

$$
E F=[0,84-(0,64 \times S T R)] \times 100[\%]
$$

Formel 5: Berechnung der Ejektionsfraktion nach L.Capan (Capan 1987)

Normal: 50-65 \% (1992)

Das Verhältnis aus Schlagvolumen und enddiastolischem Füllungsvolumen wird als Ejektionsfraktion $\quad(\mathrm{EF}) \quad$ bezeichnet. Die linksventrikuläre Ejektionsfraktionsberechnung erfolgt nach einem Algorithmus von L. Capan und wird aus dem systolischen Zeitverhältnis (STR) berechnet, die Angabe erfolgt in Prozent (Capan 1987). Die Berechnung der EF unterscheidet sich je nach genutztem Verfahren. Somit erklären sich auch die geringfügig veränderten Normwerte dieser Methodik. 


\subsubsection{Enddiastolisches Volumen / Enddiastolischer Volumenindex}

$$
E D V=\frac{\text { Schlagvolumen }(S V)}{\text { EjektionsFraktion }(E F)}[\mathrm{ml}]
$$

Formel 6: Berechnung des enddiastolischen Volumens

Das enddiastolische Volumen (EDV) beziehungsweise der enddiastolische Index (EDI) sind wie das Herzminutenvolumen, das Schlagvolumen sowie der Peak Flow abhängig vom Körpergewicht und sollten als Index verwendet werden, um die linksventrikulären Parameter vergleichen zu können.

Enddiastolischer Volumenindex

$$
E D I=\frac{\text { Enddiastolisches Volumen }(E D V)}{\text { Körperoberfläche }(K O F)}\left[\frac{\mathrm{ml}}{\mathrm{m}^{2}}\right]
$$

Formel 7: Berechnung des enddiastolischen Volumenindexes

Normal: 45-100 ml (1992)

\subsubsection{Cardiac Output/ Herzminutenvolumen}

$$
C O=\frac{(S V \times H R)}{1000}\left[\frac{l}{\min ^{2}}\right]
$$

Formel 8: Berechnung des Herzminutenvolumens

Normal: 4,5- 5 1/min (1992)

Das Herzzeitvolumen (Cardiac Output, CO) ist ein Maß für die Pumpfunktion des Herzens und beschreibt das Blutvolumen, welches in einer Minute in den Kreislauf gepumpt wird. Die Berechnung des Schlagvolumens erfolgt anhand der KubicekFormel modifiziert von Sramek auf Basis der thorakalen Impedanzänderungen (Bernstein 1986). Das Cardiac Output allein erlaubt keine Aussagen über die Effizienz der Perfusion. Zur besseren interindividuellen Vergleichbarkeit des HZV wird dieses in der Regel auf die Körperoberfläche normiert (Herzindex).

$$
C I=\frac{\text { Herzminutenvolumen }(H M V)}{\text { Körperoberfläche }(K O F)}\left[\mathrm{l} / \mathrm{min} / \mathrm{m}^{2}\right]
$$

Formel 9: Berechnung des Herzindex

Normal: $2,5 \mathrm{l} / \mathrm{min} / \mathrm{m}^{2}(1992)$ 
Der Herzindex (CI) berechnet sich aus dem Herzminutenvolumen und der Körperoberfläche und ist ein Parameter zur Beurteilung Herzleistung.

\subsubsection{Herzfrequenz}

Unter körperlicher Belastung kommt es zu einem Anstieg der Herzfrequenz (HR) bedingt durch zirkulierende Katecholamine sowie durch die vegetative Innervation des Sinusknotens (Myers and Froelicher 1993). Eine Steigerung der Herzfrequenz geschieht auf Kosten der Diastolendauer mit daraus resultierender reduzierter Füllungszeit. Die maximale Herzfrequenz und die damit verbundene Erhöhung des Herzminutenvolumens sind abhängig von verschiedenen Faktoren. Zu diesen zählen beispielsweise der Volumenstatus, das Alter, die Medikation, die Trainingszustand als auch die zugrundeliegende Erkrankung sowie klimatische Bedingungen (Trappe and Lollgen 2000).

Normal: 65- 75 [Schläge/min] (1992)

\subsubsection{Schlagvolumen/ Schlagvolumenindex}

Das Schlagvolumen (SV) wird nach der Formel von Sramek berechnet:

$S V=V E P T \times V E T \times I C[\mathrm{ml}]$

Formel 10: Berechnung des Schlagvolumens

VEPT [ml] (volume of electrically participating tissue (intrathorakal))

Die Volumenberechnung erfolgt aus Geschlecht, Größe und

Gewicht des Probanden.

VET [sec] ventrikuläre Ejektionszeit

IC $\quad\left[\mathrm{sec}^{-1}\right]$ Kontraktilitätsindex Dies entspricht dem Verhältnis von $(\mathrm{dZ} / \mathrm{dt})_{\max } / \mathrm{TFI}$

$(\mathrm{dZ} / \mathrm{dt})_{\max }[\mathrm{Ohm}-\mathrm{cm}]$ Quotient der Änderung des Widerstandes

Normal: 70-100 [ml] (1992)

Das Schlagvolumen (SV) beschreibt das Volumen, welches pro Herzkontraktion vom Herzen ausgeworfen wird. Der Ruhewert ist abhängig vom Alter, Trainingszustand, Volumenstatus sowie der Körpergröße. Neben der Herzfrequenz und der Vorlast, hat auch die Kontraktilität und die Nachlast einen Einfluss auf das Schlagvolumen. Vorlastschwankungen machen sich somit in Schwankungen des 
Schlagvolumens bemerkbar. Eine Erhöhung der Vorlast verursacht demnach einen Anstieg des Schlagvolumens und somit auch eine Steigerung der ventrikulären Ejektionszeit.

$$
S I=\frac{S V}{B S A}\left[\frac{m l}{m^{2}}\right]
$$

Formel 11: Berechnung des Schlagvolumenindexes

Normal: $\quad 30-65 \mathrm{ml} / \mathrm{m}^{2}(1992)$

Eine Berechnung des Schlagvolumenindexes erfolgt nach oben angegebener Formel, genormt auf die Körperoberfläche.

\subsubsection{Kontraktilitätsindex}

$$
I C=\frac{\left(\frac{d Z}{d t}\right) \max }{T F I(=\mathrm{Z} 0)}\left[\frac{1}{\sec }\right]
$$

Formel 12: Berechnung des Kontraktilitätsindexes

Normal: $0,033-0,060 \mathrm{sec}^{-1}(1992)$

Der Kontraktilitätsindex (IC) beschreibt das Verhältnis zwischen dem Maximum der ersten Ableitung der Impedanzänderung nach der Zeit $(\mathrm{dZ} / \mathrm{dt})_{\max }$ und dem Gesamtflüssigkeitsgehalt des Thorax $\left(\mathrm{Z}_{0}\right)$. Die Angabe der Messung erfolgt pro Sekunde. Neben dem Peak Flow ist auch der Kontraktilitätsindex ein volumenabhängiger Kontraktilitätsparameter.

\subsubsection{Thorakaler Flüssigkeitsindex}

Normal: $\quad$ Männer $33 \mathrm{Ohm}$

Frauen 27- 48 Ohm (1992)

Der thorakale Flüssigkeitsindex (TFI) ist ein Maß für die totale Gesamtimpedanz des Thorax, welche vom Thoraxquerschnitt abhängig ist. Je mehr Flüssigkeit sich im Thorax befindet, desto leitfähiger wird dieser. Dies bedeutet, dass der TFI mit steigendem Flüssigkeitsgehalt niedriger ausfällt.

Mit Hilfe des TFI-Wertes und des Kontraktilitätsindexes (IC) ist eine Differenzierung zwischen kardialem und nichtkardialem Lungenödem möglich. Der thorakale Flüssigkeitsindex ist in beiden Fällen erniedrigt, wobei der Kontraktilitätsindex bei dem kardialen Lungenödem niedrigere $(<0,035)$ und bei 
einer nicht kardialen Ursache höhere Werte aufweist (> 0,065). Eine Zunahme des TFI ist gleichzusetzen mit einer Reduktion des pulmonalen Flüssigkeitsgehaltes (Boldt, Kling et al. 1988).

\subsubsection{Akzelerationsindex}

Normal: $\quad$ Männer $0,7-1,5 \mathrm{sec}^{-2}$

$$
\text { Frauen } 0,9-1,7 \mathrm{sec}^{-2}(1992)
$$

Der Akzelerationsindex (ACI) oder Beschleunigungsindex ist ein Inotropie-Prameter des linken Ventrikels. Er beschreibt die linksventrikuläre Beschleunigung des Blutflusses während der ersten 10-20 Millisekunden (msec) nach dem Öffnen der Aortenklappe. Des Weiteren kann der ACI als Impedanzäquivalent des invasiv, mittels Herzkatheter, ermittelten $\mathrm{dp} / \mathrm{dt}_{\max }$ gesehen werden. Im Vergleich zum Peak Flow und Kontraktilitätsindex ist dieser Kontraktilitätsparameter weitaus weniger von der Vor- beziehungsweise Nachlast abhängig (Castor 1989).

\subsubsection{Ventrikuläre Ejektionszeit}

$$
V E T=\frac{(E R \times H R P)}{100}[\mathrm{msec}]
$$

Formel 13: Berechnung der ventrikulären Ejektionszeit

Normal:

\begin{tabular}{|l|l|}
\hline HR & $\begin{array}{l}\text { VET } \\
{[\mathbf{m s e c}]}\end{array}$ \\
\hline $\mathbf{5 0}$ & 360 \\
\hline $\mathbf{8 0}$ & 310 \\
\hline $\mathbf{1 2 0}$ & 250 \\
\hline $\mathbf{1 8 0}$ & 190 \\
\hline
\end{tabular}

Tabelle 10: Normwerte Herzfrequenz und ventrikuläre Ejektionszeit (1992)

Die ventrikuläre

Ejektionszeit (VET) ist abhängig von der Herzfrequenz und der Ejektionsfraktion.

Der Absolutwert hat keine spezifische diagnostische oder therapeutische Relevanz. Es erfolgt dennoch die Berechnung, da sie dem Anwender die Kalkulation der PreEjektionsperiode (PEP) erlaubt.

$$
P E P=V E T \times S T R
$$




\subsubsection{Auswurfverhältnis/ Ejektionsverhältnis}

$E R=\frac{V E T}{H R P}[\%]$

Formel 15: Berechnung des Ejektionsverhältnisses

$$
H R P=\frac{60}{H R}[\mathrm{sec}]
$$

Formel 16: Berechnung der Herzfrequenzperiode

Normal:

\begin{tabular}{|c|c|c|c|}
\hline HR [bpm] & VET [msec] & HRP [msec] & ER [\%] \\
\hline $\mathbf{5 0}$ & 360 & 1200 & 30 \\
\hline $\mathbf{8 0}$ & 310 & 750 & 41 \\
\hline $\mathbf{1 2 0}$ & 250 & 500 & 50 \\
\hline $\mathbf{1 8 0}$ & 190 & 330 & 57 \\
\hline
\end{tabular}

Tabelle 11: Normwerte Herzfrequenz, VET, Herzfrequenzperiode, ER (1992)

Der Einfluss der Herzfrequenz auf die ventrikuläre Austreibungszeit wird durch die Normierung auf die Herzfrequenzperiode (HRP) aufgehoben. Der wichtigste diagnostische und therapeutische Wert des Ejektionsverhältnisses (ER) ist die Reaktion auf Veränderungen in der Vorlast. Daher kann dieser Parameter auch als Vorlast-Index bezeichnet werden. Eine Erhöhung der Vorlast verursacht einen Anstieg des Enddiastolischen Volumens (EDV) und beim gesunden Herzen eine Erhöhung des Schlagvolumens (SV) was wiederum eine verlängerte Auswurfzeit nach sich zieht.

\subsubsection{Systolisches Zeitverhältnis}

$$
S T R=\frac{P E P}{V E T}[\%]
$$

Formel 17: Berechnung des systolisches Zeitverhältnisses

Normal: 30-50 \% (1992)

Das systolische Zeitverhältnis (STR) wird von der Messung des systolischen Zeitintervalls, der Pre-Ejektionsperiode (PEP) zur ventrikulären Austreibungszeit (VET), abgeleitet. Es beschreibt das Verhältnis der elektrischen zur mechanischen 
Systole. Die Pre-Ejektionsperiode entspricht der Dauer der elektrischen Systole und stellt die Dauer der isovolumetrischen Kontraktion des Ventrikels dar.

\subsection{Parameter der Spiroergometrie}

Es handelt sich bei der Spiroergometrie um ein Verfahren, bei welchem unter Belastung Herz-Kreislaufparameter, Atmung sowie die kardiopulmonale Belastbarkeit beurteilt werden. Aufgrund der kontinuierlichen Ermittlung der Sauerstoffaufnahme sowie der Kohlendioxidabgabe, als auch der Erhebung von Herzfrequenz und Atemstromstärke, wird eine Differenzierung der leistungslimitierenden Organstörung möglich (Wonisch 2003).

Die Spiroergometrie kann im Zuge der thoraxchirurgischen Eingriffe zur präbeziehungsweise postoperativen Risikoabschätzung herangezogen werden (Goeckenjan, Sitter et al. 2010) (Westhoff 2007). Sie nimmt hinsichtlich der prognostischen Einschätzung sowie in der Funktionsbeurteilung von Erkrankungen wie der pulmonalen Hypertonie, einen hohen Stellenwert ein (Westhoff 2011). Gleiches gilt für die Beurteilung des Verlaufs und die Bewertung der durchgeführten Therapie.

Die mittels Spiroergometrie erhobenen Parameter sind trotz Normierung der Protokolle auch abhängig von der Art der durchgeführten Belastung. Fahrradergometrische Belastungstests mit ansteigender Belastung finden bei den herkömmlichen klinischen Fragestellungen ihre Verwendung. Hierbei wird zwischen Belastungstest mit stufenförmiger Steigerung (drei bis fünf-minütliche Belastungssteigerung) und kontinuierlich ansteigender Belastung (Rampenbelastung) unterschieden. Die Nutzung der Rampenbelastung führt im Gegensatz zur stufenweisen Belastung $\mathrm{zu}$ einer schnelleren Ermittlung der maximalen Leistungsfähigkeit (Myers, Buchanan et al. 1991). Für die Gesamtdauer der Belastung wird eine Zeit von circa zehn Minuten empfohlen. Kürzere Belastungszeiten führen zu einer Diskrepanz zwischen Sauerstoffaufnahme und Arbeitstempo, wohingegen eine Verlängerung zu keinem adäquaten Anstieg führt (Myers and Froelicher 1993). Während der Belastungsuntersuchung erfolgten die kardiale Überwachung mittels eines 12-Kanal-EKGs, sowie die Ermittlung des Blutdrucks und der respiratorischen Parameter.

Nachfolgend eine Übersicht der Kenngrößen der Spiroergometrie hinsichtlich des Gasaustausches, sowie der Ventilation und dem Belastungsverhalten. 


\begin{tabular}{|c|c|c|}
\hline \multicolumn{3}{|c|}{ Belastung-/ Leistungsparameter } \\
\hline & Herzfrequenz & {$[1 / \mathrm{min}]$} \\
\hline & Blutdruck & {$[\mathrm{mmHg}]$} \\
\hline & Leistung & [Watt] \\
\hline & Elektrokardiogramm & \\
\hline \multicolumn{3}{|c|}{ Parameter des Gasaustausches } \\
\hline \multirow[t]{2}{*}{ ermittelt } & Sauerstoffaufnahme $\dot{\mathrm{VO}}_{2}$ & {$[\mathrm{ml} / \mathrm{min}]$} \\
\hline & Kohlendioxidabgabe $\dot{\mathrm{VCO}}_{2}$ & {$[\mathrm{ml} / \mathrm{min}]$} \\
\hline \multirow[t]{4}{*}{ abgeleitet } & Sauerstoffaufnahme pro kg Körpergewicht $\dot{\mathrm{VO}}_{2} / \mathrm{kg}$ & {$[\mathrm{ml} / \mathrm{min} / \mathrm{kg}]$} \\
\hline & Anaerobe Schwelle VAT & {$[\mathrm{ml} / \mathrm{min}]$} \\
\hline & Respiratorischer Quotient $\mathrm{RQ}=\dot{\mathrm{V}} \mathrm{CO}_{2} / \dot{\mathrm{VO}}_{2}$ & \\
\hline & Sauerstoffpuls O2-Puls $=\dot{\mathrm{VO}} 2 / \mathrm{HF}$ & {$[\mathrm{ml}]$} \\
\hline \multicolumn{3}{|c|}{ Ventilationsparameter } \\
\hline \multirow[t]{5}{*}{ ermittelt } & Atemzugvolumen VT & {$[\mathrm{ml}]$} \\
\hline & Atemfrequenz AF & {$[1 / \mathrm{min}]$} \\
\hline & Atemreserve & {$[1 / \mathrm{min}]$} \\
\hline & Endexpiratorischer Sauerstoffpartialdruck $\mathrm{P}_{\mathrm{ET}} \mathrm{O}_{2}$ & {$[\mathrm{kPa}$} \\
\hline & Endexpiratorischer Kohlendioxidpartialdruck $\mathrm{P}_{\mathrm{ET}} \mathrm{CO}_{2}$ & {$[\mathrm{kPa}$} \\
\hline \multirow[t]{7}{*}{ abgeleitet } & Atemäquivalent für Sauerstoff $\mathrm{EqO} 2=\dot{\mathrm{VE}} / \dot{\mathrm{V}}_{2}$ & \\
\hline & Atemäquivalent für Kohlendioxid $\mathrm{EqCO}_{2}=\dot{\mathrm{VE}} / \dot{\mathrm{VCO}}_{2}$ & \\
\hline & Atemminutenvolumen $\dot{V} E$ & {$[1 / \mathrm{min}]$} \\
\hline & Totraum VD & {$[\mathrm{ml}]$} \\
\hline & Totraumventilation $\mathrm{V}_{\mathrm{D}} / \mathrm{V}_{\mathrm{T}}$ & \\
\hline & $\begin{array}{l}\text { Endexpiratorisch alveoläre/arterielle } \\
\text { Partialdruckdifferenz für Sauerstoff } \mathrm{AaDO}_{2}\end{array}$ & {$[\mathrm{kPa}]$} \\
\hline & $\begin{array}{l}\text { Endexpiratorisch alveoläre/arterielle } \\
\text { Partialdruckdifferenz für Kohlendioxid } \mathrm{D}_{\mathrm{Aa}} \mathrm{CO}_{2}\end{array}$ & {$[\mathrm{kPa}]$} \\
\hline
\end{tabular}
Tabelle 12: Parameter der Spiroergometrie

\subsubsection{Herzfrequenz/ Blutdruck}

Die Berechnung des Sollwertes der maximalen Herzfrequenz unter Belastungsbedingungen erfolgt nach der Formel 220-Alter. Für ältere Patienten wird jedoch die Ermittlung nach folgender Formel: (210- 0,65xAlter) oder (202-0,72 [Alter]) laut Richtlinien der American Thoracic Society empfohlen (Ross 2003). Ein unzureichender Anstieg der Herzfrequenz, nach Ausschluss medikamentöser Ursachen (Betablocker), kann hinweisend auf eine reduzierte linksventrikuläre Pumpfunktion sein oder auch auf eine Störung im Bereich des Sinusknotens hindeuten (Ellestad and Wan 1975).

Während der Belastung steigt der Blutdruck physiologisch kontinuierlich an. Während der systolische Wert bis zu 200mmHg ansteigen kann, zeigen sich im diastolischen Bereich weitaus geringere Schwankungen, verglichen mit dem 
Ausgangswert von etwa $\pm 10 \mathrm{mmHg}$ (White, Lund-Johansen et al. 1990). Auf eine schwerwiegende myokardiale Störung der Pumpfunktion beziehungsweise auf eine koronare Mehrgefäßerkrankung kann ein unzureichender systolischer Blutdruckanstieg $(<120 \mathrm{mmHg})$ sowie ein Absinken unterhalb der Vorwerte hinweisen (Lele, Scalia et al. 1994). Bei Patienten mit pulmonaler Hypertonie ist ein fehlender Blutdruckanstieg ein prognostisch ungünstiges Zeichen (Dumitrescu and Rosenkranz 2008).

\subsubsection{Sauerstoffaufnahme/ Kohlendioxidabgabe}

Der Parameter $\dot{\mathrm{VO}}_{2 \max }$, welcher die höchste Sauerstoffaufnahme zum Zeitpunkt der maximalen Belastung repräsentiert, spiegelt die Leistungsfähigkeit des kardiopulmonalen Systems wider. Er gibt an, wie viele Milliliter Sauerstoff pro Zeiteinheit von dem eingeatmeten Gasvolumen extrahiert werden. Die Angabe der $\dot{\mathrm{VO}}_{2}$ erfolgt in $[\mathrm{ml} / \mathrm{min}]$, zur einheitlichen Beurteilung erfolgt eine Anpassung auf das Körpergewicht $[\mathrm{ml} / \mathrm{min} / \mathrm{kg}]$. Im Leerlauf kann die Berechnung der Sauerstoffaufnahme $\dot{\mathrm{V}} \mathrm{O}_{2}$ nach der Formel $\dot{\mathrm{V}}_{2}=150+(6 \mathrm{xGewicht}$ [kg]) erfolgen (Wasserman 2005). Die Ermittlung der Sollwerte für $\dot{V}_{2 \max }$ ist gewichtsabhängig. Mit Hilfe des Wasserman-Algorithmus kann eine orientierende Ermittlung für idealgewichtige Patienten nach der Formel $\left(\dot{\mathrm{V}}_{2 \max }=\right.$ Größe $[\mathrm{cm}]-$ Alter [Jahre] x F) erfolgen (der Faktor F beträgt bei Frauen 14 und bei Männern 20), anderenfalls sollten umfangreichere Berechnungen für die Sollwerte nach Wasserman genutzt werden (Westhoff 2011). Wie in der Formel ersichtlich, sind neben der Körpergröße und dem jeweiligen Belastungsprogramm, das Geschlecht sowie das Alter und das Körpergewicht weitere beeinflussende Parameter der maximalen Sauerstoffaufnahme (Winter, Gitt et al. 1994).

Die Kohlendioxidabgabe $\left(\dot{\mathrm{V}} \mathrm{CO}_{2}\right)$ beschreibt die Menge an Kohlendioxid, welche pro Zeiteinheit abgeatmet wird. Während hoher Belastungsintensitäten kommt es, bedingt durch das Anfluten von Laktat, zu einer erhöhten $\mathrm{CO}_{2}$-Abatmung. Dieses dient der respiratorischen Kompensation einer unter Belastung entstehenden metabolischen Azidose (Wonisch 2003). Die Normwerte der Kohlendioxidabgabe sind, ebenso wie die der Sauerstoffaufnahme, von dem Körpergewicht und -größe, von Alter sowie Geschlecht abhängig. 


\subsubsection{Respiratorischer Quotient}

Der respiratorische Quotient (RQ) setzt sich aus dem Verhältnis von Kohlendioxidabgabe $\left(\dot{\mathrm{VCO}}_{2}\right)$ zu Sauerstoffaufnahme $\left(\dot{\mathrm{V}}_{2}\right)$ zusammen. Die Normwerte des respiratorichen Quotienten sind abhängig von den zur Energiegewinnung genutzten Substraten. Somit kann dieser zur Abschätzung der Kohlenhydrat- sowie Eiweiß- und Fettverwertung genutzt werden. Bei der reinen Kohlenhydratnutzung entspricht der $\mathrm{RQ}=1$. Eine ausschließlich auf Eiweiß beruhende Ernährung führt $\mathrm{zu}$ einen $\mathrm{RQ}=0,81$. Eine reine Fettverwertung führt dahingegen zu einem RQ von circa 0,7. Während einer normalen Ernährung kommt es allerdings zu einer Durchmischung dieser drei Brennstoffe. Der RQ ist somit unter Ruhe vom jeweiligen Ernährungs- sowie Trainingszustand abhängig (Putman, Spriet et al. 1993) (Poehlman, McAuliffe et al. 1990).

\subsubsection{Anaerobe Schwelle}

Die anaerobe Schwelle (AT) erlaubt bei Patienten mit bestehenden kardiovaskulären Erkrankungen sowie bei Gesunden ohne maximale Ausbelastung sowie willentliche Beeinflussung eine objektive Beurteilung der aeroben Leistungsfähigkeit (Wasserman 1988; Itoh, Taniguchi et al. 1990; Jennings and Esler 1990). Zur Bestimmung der anaeroben Schwelle gibt es mehrere Möglichkeiten. Nach Beaver kann die ventilatorische Schwellenbestimmung indirekt mittels der V-slope-Methode erfolgen (Beaver, Wasserman et al. 1986). Hierzu wird die Kohlendioxidabgabe zur Sauerstoffaufnahme in einem Koordinatensystem aufgetragen und anschließend die AT anhand der Änderung der Anstiegssteilheit von Kohlendioxidabgabe gegenüber der Sauerstoffaufnahme bestimmt (Wasserman, Stringer et al. 1994). Eine weitere Ermittlung der AT ist über die Atemäquivalente möglich (Anstieg des $\dot{\mathrm{VE}} / \dot{\mathrm{V}} \mathrm{CO}_{2}$ bei gleichbleibendem $\dot{\mathrm{V} E} / \dot{\mathrm{V}}_{2}$ ). Durch die beginnende Laktatazidose, oberhalb dieser Schwelle, kommt es zu einer erhöhten Freisetzung von $\mathrm{CO}_{2}$ aufgrund der Bikarbonatpufferung, dies führt zu einem erhöhten Anstieg der Kohlendioxidabgabe im Vergleich zur Sauerstoffaufnahme. Anhand der direkten Messungen des Laktatanstieges im arteriellen Blut resultiert die Festlegung der anaeroben Schwelle nach Wasserman (Wasserman, Stringer et al. 1994). Bei einer bestehenden ventilatorischen Einschränkung ist ein Erreichen der AT häufig nicht möglich. Bei Patienten mit pulmonaler Hypertonie zeigt sich durch einen eingeschränkten 
Sauerstofftransport eine reduzierte anaerobe Schwelle (Dumitrescu and Rosenkranz 2008).

\subsubsection{Sauerstoffpuls}

Der Sauerstoffpuls kann als Korrelat des Schlagvolumens angesehen werden (Sun, Hansen et al. 2001). Normwerte liegen unter Ruhebedingungen im Bereich von 4-6ml/Schlag und steigen unter maximaler Belastung auf etwa 10-20 $\mathrm{ml} / \mathrm{Schlag}$ an (Wonisch 2003). Er setzt sich zusammen aus dem Quotienten von Sauerstoffaufnahme $\left(\dot{\mathrm{V}}_{2}\right)$ und Herzfrequenz. Das Herzzeitvolumen ergibt sich wiederum aus dem Schlagvolumen und der Herzfrequenz. Somit kann eine Aussage über die kardiale Funktion anhand des zeitlichen Verlaufs der Sauerstoffpulskurve getätigt werden (Westhoff 2011). Zu erniedrigten Werten können beispielsweise eine geringe Oxygenierung, reduzierte Hämoglobinwerte oder das Vorhandensein eines Rechts-Links-Shunts beitragen (Wonisch 2003). Der Sauerstoffpuls ist abhängig vom Schlagvolumen, welches bei einer bestehenden Herzinsuffizienz reduziert sein kann. Daher stellt dieser unter Belastungsbedingungen einen bedeutenden Parameter bezüglich der myokardialen Funktion dar. Erniedrigte Werte sind vor allem bei Patienten mit einer reduzierten Auswurfleistung und frühen Ausbildung eines Plateaus unter steigender Belastung anzutreffen (Smith, Johnson et al. 1993). Die Einnahme von Betablockern bei bestehender Herzinsuffizienz führt, bedingt durch die reduzierte Herzfrequenz, zu höheren Absolutwerten des Sauerstoffpulses auf der jeweiligen Belastungsstufe (Wonisch, Hofmann et al. 2002).

\subsubsection{Atemzugvolumen/ Atemfrequenz und Atemreserve}

Die Atemfrequenz (AF) definiert sich über die Anzahl der Atemzüge pro Minute. Die Normwerte liegen unter Ruhebedingungen bei etwa 10 bis 15 Atemzüge pro Minute, das Atemzugvolumen liegt bei circa 400 bis $600 \mathrm{ml}$, umgerechnet auf das Körpergewicht bei etwa 7 bis $10 \mathrm{ml} / \mathrm{kg}$ (Rühle 2008). Die klinische Relevanz ist in der Beurteilung der pulmonalen Leistungslimitierung $\mathrm{zu}$ sehen. Eine flache und schnelle Atmung ist hinweisend auf eine drohende ventilatorische Erschöpfung beziehungsweise eine Ausschöpfung der vorhandenen Atemreserven (Wasserman 1994). Das Atemzugvolumen (VT) entspricht dem Luftvolumen, welches pro Atemzug ein- beziehungsweise ausgeatmet wird. Gesunde sind in der Lage, ihr Atemminutenvolumen mit ansteigender Belastung zunächst über eine Erhöhung des Atemzugvolumens zu regulieren. Im weiteren Verlauf der Belastung kommt es zu 
einer Zunahme der Atemfrequenz (Wasserman 1994). Wenn das Atemzugvolumen circa 50-60\% der Vitalkapazität beträgt, ist eine Ausbelastung erreicht (Rühle 2008).

Das Atemzugvolumen sowie die Atemfrequenz sind neben dem Atemminutenvolumen und der Atemreserve (BR) wesentliche Parameter zur Einschätzung obstruktiver als auch restriktiver Atemmuster. Die Atemreserve lässt sich aus der Differenz des Atemgrenzwertes (MVV-maximal voluntary ventilation) $\left(\mathrm{FEV}_{1}\right.$ x 35) und dem aktuellen Atemminutenvolumen berechnen, es sollten $15 \mathrm{l} / \mathrm{min}$ beziehungsweise 10-40\% der MVV nicht unterschritten werden (Rühle 2008). Erfolgt eine Unterschreitung des MVV ist dies hinweisend auf eine ventilatorische Limitierung.

\subsubsection{Atemäquivalente für Sauerstoff $\mathrm{EqO}_{2}$ und $\mathrm{Kohlendioxid} \mathrm{EqCO}_{2}$}

Die Atemäquivalente beschreiben die erforderliche Ventilation, welche zur Aufnahme beziehungsweise Abgabe einer bestimmten Menge Sauerstoff oder Kohlendioxid erforderlich sind (Rühle 2008). An der anaeroben Schwelle wird der niedrigste Wert für $\mathrm{EqO}_{2}$ gemessen. Im Zuge der Belastung kommt es zu einem anfänglichen Anstieg von $\mathrm{EqO}_{2}$ bedingt durch die Hyperventilation, um das durch die Laktatpufferung entstehende zusätzliche Kohlendioxid zu entfernen. Der anschließende Anstieg des EqCO2 entspricht nach Wasserman dem respiratorischen Kompensationspunkt. Am aerob/ anaeroben Übergang liegen die Normalwerte für $\mathrm{EqO}_{2}$ und $\mathrm{EqCO} 2$ bei $25 \pm 3$ (Westhoff 2011).

\subsection{Datenerfassung und Statistik}

Die Zusammenführung der Daten der kardialen Bioimpedanzmessung erfolgte zusammen mit den Daten der Spiroergometrie, der Echokardiographie, der Hämodynamik sowie den Patientendaten in einer Microsoft ${ }^{\circledR}$-Excel-Datenbank. Die statistischen Berechnungen wurden mit Aabel (Gigawiz) 3.0.3 unter Mac OS X durchgeführt. Ausgeschlossen wurden Daten außerhalb eines Intervalls von drei Standardabweichungen (SD) um den Mittelwert $(\mu \pm 3 \sigma)$. Falls nicht anders angegeben, erfolgen Darstellung und Angabe der Werte als Mittelwert \pm Standardabweichung (SD). Die Testung der Parameter auf Normalverteilung erfolgte mit dem Shapiro-Wilk-Test, Gruppenvergleiche mit dem Mann-Whitney-U-Test, Korrelationen mit Kendall's Tau. Ein p-Wert kleiner 0,05 wurde als statistisch signifikant angesehen. 


\section{Ergebnisse}

\subsection{Qualitätskontrolle}

\begin{tabular}{|l|c|}
\hline & Mittlere Schwankungsbreite [\%] \\
\hline PFI & $6,8 \pm 3,15$ \\
\hline EF & $2,92 \pm 2,67$ \\
\hline EDI & $4,18 \pm 1,65$ \\
\hline CI & $5,42 \pm 2,05$ \\
\hline HR & $2,81 \pm 2,46$ \\
\hline SI & $3,39 \pm 1,71$ \\
\hline \multicolumn{2}{|c|}{ Tabelle 13: Qualitätskontrolle $(n=6)$} \\
\hline
\end{tabular}

Anhand einer Stichprobe von sechs Patienten wurde eine interne Qualitätskontrolle unter Ruhebedingungen durchgeführt. Es erfolgte die Ermittlung der mittleren Schwankungsbreite für die Parameter: Peak Flow Index, Ejektionsfraktion, enddiastolischer Volumenindex, Herzindex, Herzfrequenz sowie für den Schlagvolumenindex. Hierbei zeigte sich für alle Parameter eine geringe intraindividuelle Variabilität. Die höchste Schwankungsbreite wies der Peak Flow Index (PFI) mit 6,8\% und einer Standardabweichung von 3,15 auf.

\subsection{Basischarakteristika}

\begin{tabular}{|c|c|c|c|c|}
\hline \multirow{2}{*}{ Basisparameter } & Kontrollgruppe & Patienten & PAH & СТЕРН \\
\hline & $\mathrm{MW} \pm \mathrm{SD}$ & $\mathrm{MW} \pm \mathrm{SD}$ & $\mathrm{MW} \pm \mathrm{SD}$ & $\mathrm{MW} \pm \mathrm{SD}$ \\
\hline Anzahl & 19 & 24 & 13 & 11 \\
\hline [Jahre] & $47,6 \pm 14,7$ & $57,1 \pm 17,4(*)$ & $53,2 \pm 18$ & $61,7 \pm 16,3 \#$ \\
\hline Geschlecht $[\mathrm{m} / \mathrm{w}]$ & $11 / 8$ & $5 / 19$ & $1 / 12$ & $4 / 7$ \\
\hline Körpergewicht [kg] & $79 \pm 14,5$ & $75,5 \pm 19,2$ & $74,3 \pm 23,8$ & $77 \pm 13$ \\
\hline Körpergröße [cm] & $175,6 \pm 8,9$ & $165,5 \pm 8,1 *$ & $163,8 \pm 6,9 \#$ & $167,4 \pm 9,4 \#$ \\
\hline Körperoberfläche $\left[\mathrm{m}^{2}\right]$ & $1,94 \pm 0,22$ & $1,81 \pm 0,24^{*}$ & $1,78 \pm 0,29 \#$ & $1,84 \pm 0,17$ \\
\hline BMI $\left[\mathrm{kg} / \mathrm{m}^{2}\right]$ & $25,5 \pm 3,04$ & $27,5 \pm 6,06$ & $27,3 \pm 6,85$ & $27,7 \pm 5,30$ \\
\hline WHO-FC & 0 & II - III & II - III & II - III \\
\hline
\end{tabular}

Tabelle 14: Übersicht der Basischarakteristika

In der Kontrollgruppe befanden sich 19 gesunde Probanden. Das gesamte Patientenkollektiv umfasste 24 Patienten, von welchen 13 an einer pulmonal arteriellen Hypertonie (PAH) und 11 an einer chronisch thromboembolischen pulmonalen Hypertonie (CTEPH) litten. 


\section{Studienteilnehmer}

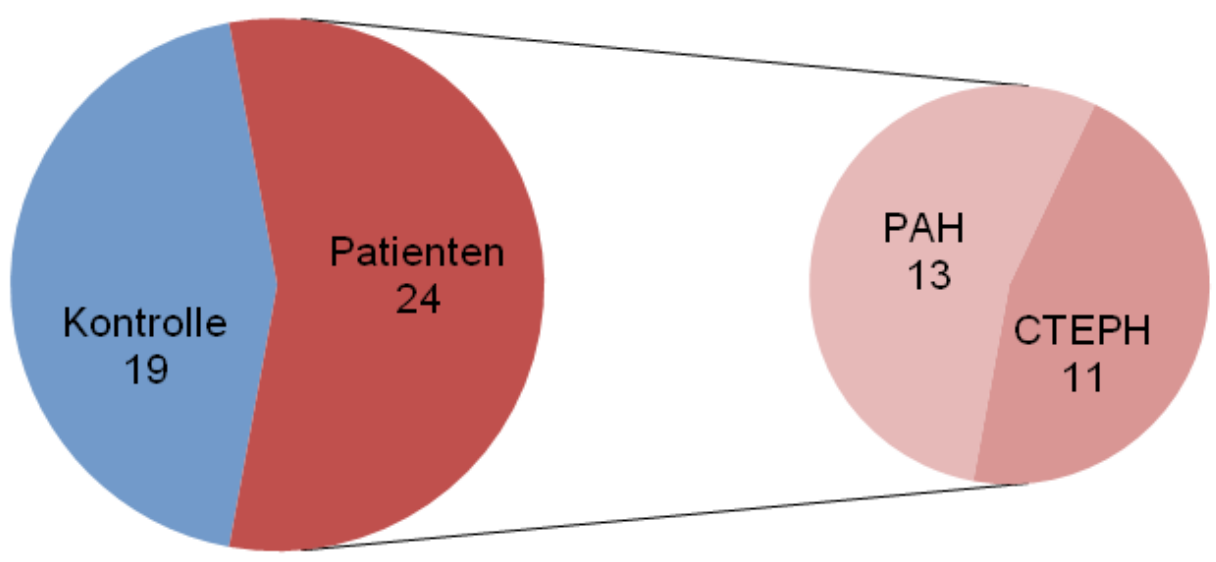

Abbildung 3: Verteilung und Anzahl der Studienteilnehmer

Das Alter aller Studienteilnehmer lag im Mittel bei $53 \pm 17$ Jahren, der jüngste Studienteilnehmer war zum Zeitpunkt der Untersuchung 21 und der älteste 80 Jahre.

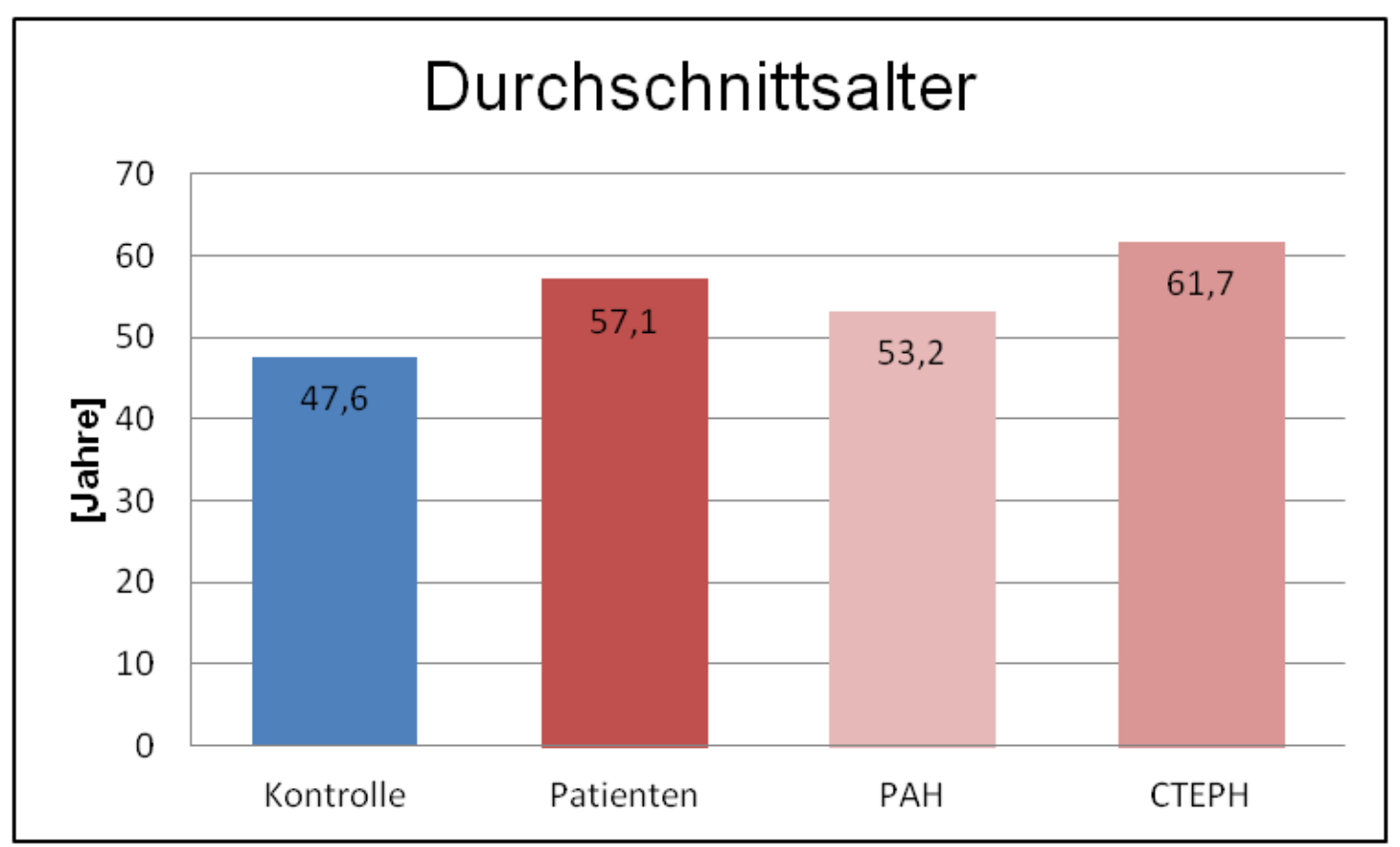

Abbildung 4: Durchschnittsalter der Studienteilnehmer 
4.3 Medikation zum Zeitpunkt der Untersuchung

\begin{tabular}{|l|r|r|r|r|}
\hline \multirow{2}{*}{$\begin{array}{l}\text { Medikation } \\
\mathrm{n}\end{array}$} & Kontrollgruppe & Patienten & \multicolumn{1}{c|}{ PAH } & \multicolumn{1}{c|}{ CTEPH } \\
\cline { 2 - 5 } & 19 & 24 & 13 & 11 \\
\hline PDEI & 0 & $13(54 \%)$ & $11(85 \%)$ & $2(18 \%)$ \\
\hline ERA & 0 & $11(46 \%)$ & $8(62 \%)$ & $3(27 \%)$ \\
\hline PC & 0 & $6(25 \%)$ & $6(46 \%)$ & 0 \\
\hline CAA & 0 & $2(8 \%)$ & $1(8 \%)$ & $1(9 \%)$ \\
\hline
\end{tabular}

Tabelle 15: Medikation zum Untersuchungszeitpunkt

\begin{tabular}{|r|r|r|r|r|}
\hline \multirow{2}{*}{$\begin{array}{l}\text { Anzahl der Medikation } \\
\text { n }\end{array}$} & Kontrollgruppe & Patienten & \multicolumn{1}{|c|}{ PAH } & CTEPH \\
\cline { 2 - 5 } & 19 & 24 & 13 & 11 \\
\hline 0 & $19(100 \%)$ & $7(29 \%)$ & 0 & $7(64 \%)$ \\
\hline 1 & 0 & $6(25 \%)$ & $4(31 \%)$ & $2(18 \%)$ \\
\hline 2 & 0 & $7(29 \%)$ & $5(38 \%)$ & $2(18 \%)$ \\
\hline 3 & 0 & $4(17 \%)$ & $4(31 \%)$ & 0 \\
\hline
\end{tabular}

Tabelle 16: Anzahl der Medikation zum Untersuchungszeitpunkt

In den Tabellen ist die Medikation der Patienten zum Untersuchungszeitpunkt nachzuvollziehen. Mehr als die Hälfte aller Patienten nahm bei der Datenerhebung Phosphodieserase 5-Inhibitoren (PDEI) ein, 46\% Endothelin-Rezeptor-Antagonisten (ERA). Auf die Prostanoide und Calciumantagonisten fiel nur ein geringer Prozentsatz. Die meisten PAH-Patienten wurden mit einer Kombinationstherapie behandelt.

\subsection{Vergleich der Sauerstoffaufnahme und Leistung (spiroergometrische Parameter)}

In der Auswertung der maximalen Sauerstoffaufnahme und maximalen Leistung erfolgte eine zusammengefasste Betrachtung der Patienten mit pulmonal arterieller Hypertonie und chronisch thromboembolischer pulmonaler Hypertonie im Vergleich zur gesunden Kontrollgruppe.

\begin{tabular}{|c|c|c|}
\hline & Kontrollgruppe & Patienten \\
\hline & $\mathrm{MW} \pm \mathrm{SD}$ & $\mathrm{MW} \pm \mathrm{SD}$ \\
\hline Maximale Leistung [Watt] & $185,4 \pm 85,4$ & $53,7 \pm 35,0^{*}$ \\
\hline $\begin{array}{l}\text { Maximale Sauerstoffaufnahme } \\
\left(\dot{\mathrm{V}} \mathrm{O}_{2} \text { max }\right)[\mathrm{ml} / \mathrm{min}]\end{array}$ & $2157,7 \pm 918$ & $850,8 \pm 418 *$ \\
\hline
\end{tabular}

Tabelle 17: maximale Sauerstoffaufnahme und Leistung der Patienten im Vergleich zur Kontrollgruppe 


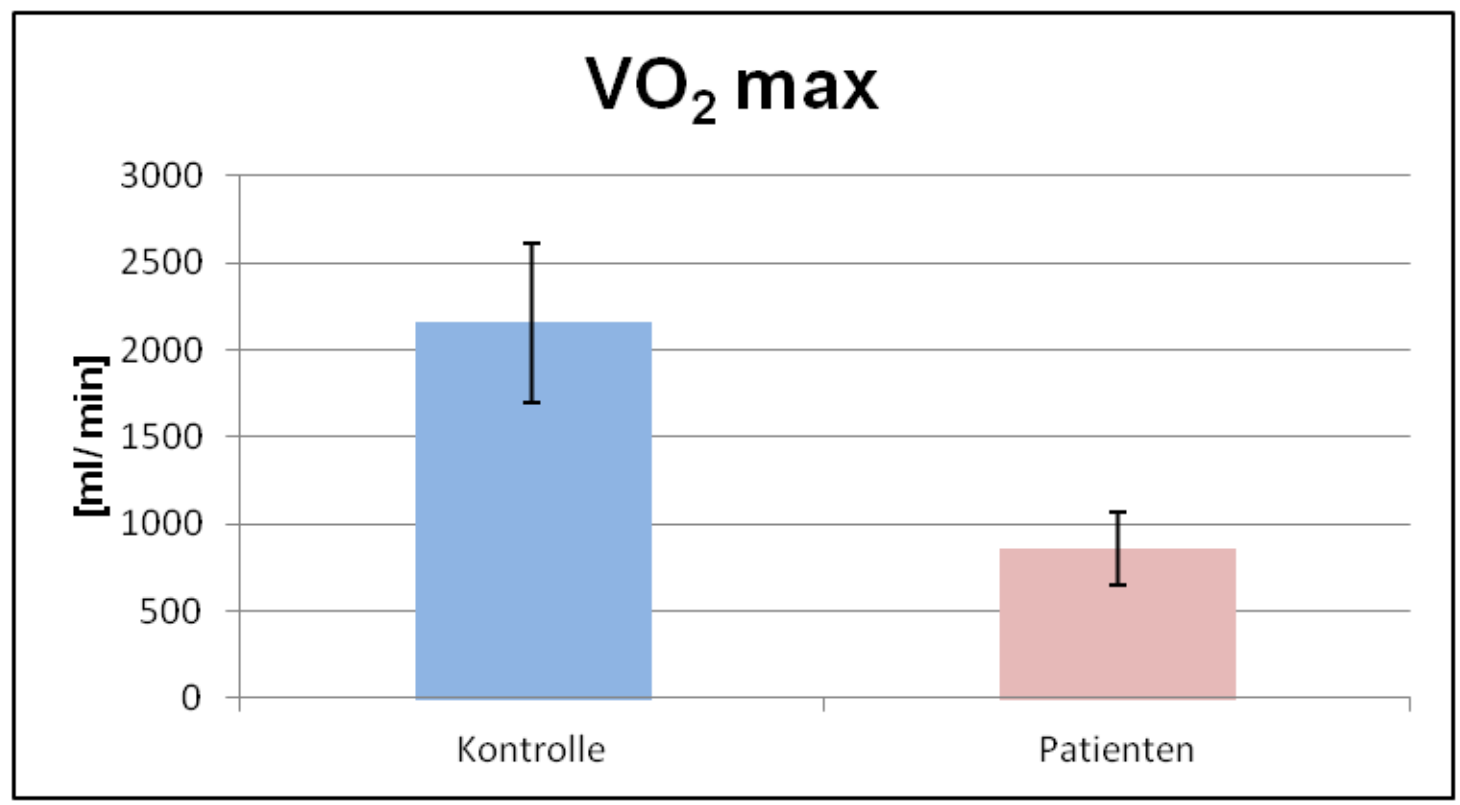

Abbildung 5: Vergleich der maximalen Sauerstoffaufnahme zwischen Patienten- und Kontrollgruppe

Die Sauerstoffaufnahme im Bereich der maximalen Belastung $\left(\dot{\mathrm{V}}_{2 \max }\right)$ beschreibt die individuelle Leistungsfähigkeit. Die Patienten wiesen eine weitaus geringere maximale Sauerstoffaufnahme $\left(\dot{\mathrm{V}}_{2} \max \right)(850,8 \pm 418 \mathrm{ml} / \mathrm{min})$ im Vergleich zur gesunden Kontrollgruppe auf $(2157,7 \pm 918 \mathrm{ml} / \mathrm{min})$. Die $\dot{\mathrm{VO}}_{2} \mathrm{max}$ der Patienten war im Vergleich zur gesunden Kontrolle um etwa $60 \%$ reduziert.

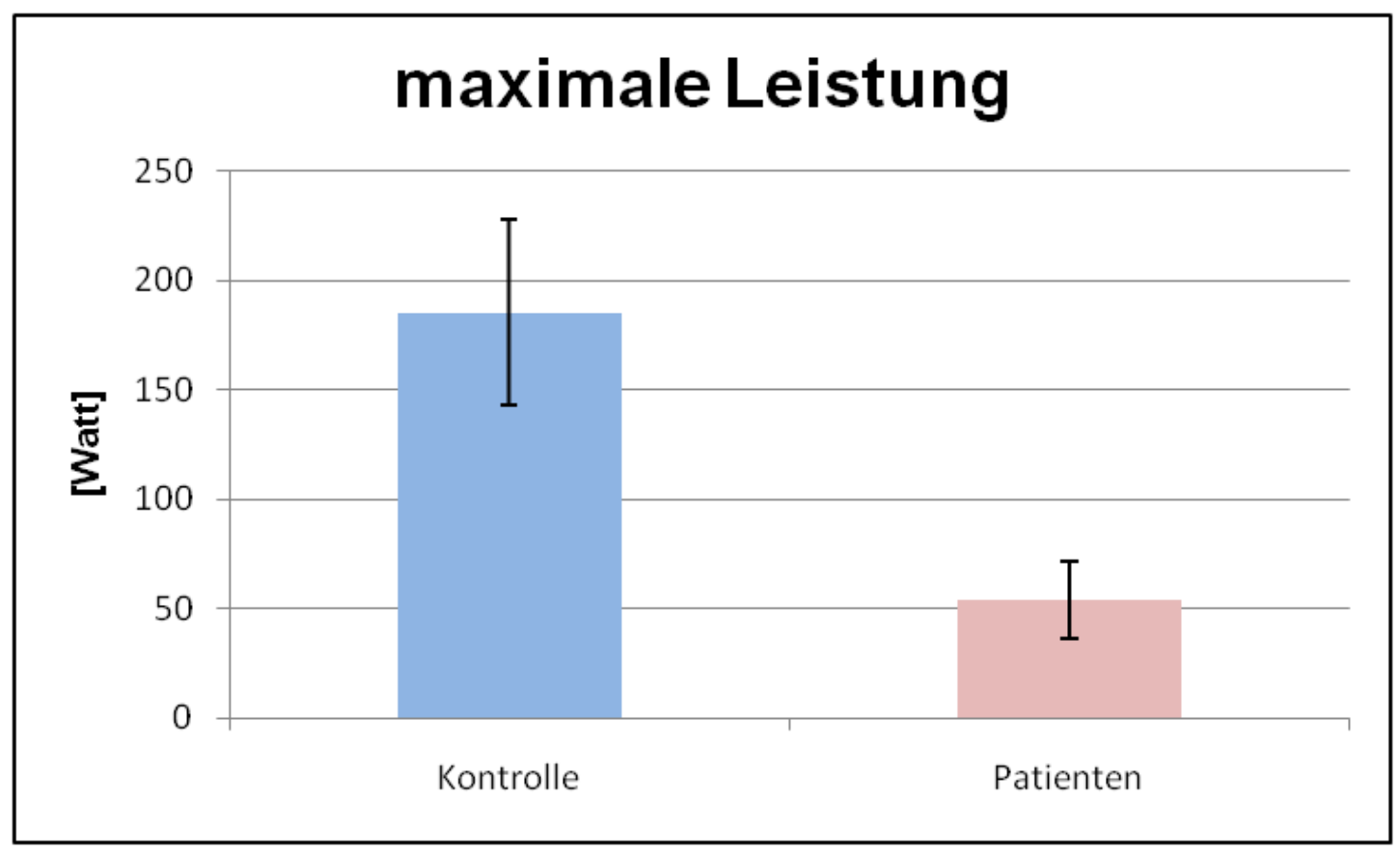

Abbildung 6: Vergleich der maximalen Leistung zwischen Patienten- und Kontrollgruppe

Wie zu erwarten zeigten die Untersuchungen, dass das Patientenkollektiv weitaus geringere maximale Belastungsstufen $(53,7 \pm 35,0$ Watt) im Vergleich zur gesunden 
Kontrollgruppe $(185,4 \pm 85,4$ Watt) erreichen konnte. Die Patienten erbrachten in etwa $30 \%$ der Leistung der gesunden Probanden.

\subsubsection{Vergleich der Leistungsfähigkeit an den jeweiligen \\ Belastungszeitpunkten}

\begin{tabular}{|l|c|c|c|c|}
\hline \multirow{2}{*}{ Watt } & Kontrollgruppe & Patienten & PAH & CTEPH \\
\cline { 2 - 5 } & $\mathrm{MW} \pm \mathrm{SD}$ & $\mathrm{MW} \pm \mathrm{SD}$ & $\mathrm{MW} \pm \mathrm{SD}$ & $\mathrm{MW} \pm \mathrm{SD}$ \\
\hline $\mathrm{BL}$ & $0,00 \pm 0,00$ & $0,00 \pm 0,00$ & $0,00 \pm 0,00$ & $0,00 \pm 0,00$ \\
\hline $\mathrm{UP}$ & $0,00 \pm 0,00$ & $0,00 \pm 0,00$ & $0,00 \pm 0,00$ & $0,00 \pm 0,00$ \\
\hline $\mathrm{EE}$ & $52,17 \pm 29,37$ & $19,68 \pm 19,55^{*}$ & $17,55 \pm 8,14 \#$ & $21,82 \pm 26,95 \#$ \\
\hline $\mathrm{AT}$ & $135,72 \pm 60,75$ & $48,44 \pm 31,63^{*}$ & $48,50 \pm 16,20 \#$ & $48,40 \pm 41,05 \#$ \\
\hline $\mathrm{SM}$ & $173,00 \pm 79,90$ & $52,43 \pm 34,08^{*}$ & $51,92 \pm 24,50 \#$ & $53,00 \pm 43,53 \#$ \\
\hline RC & $0,00 \pm 0,00$ & $0,00 \pm 0,00$ & $0,00 \pm 0,00$ & $0,00 \pm 0,00$ \\
\hline $\begin{array}{l}{ }^{*} \mathrm{p}<0,05 \text { PH vs. Kontrolle } \\
\# \mathrm{p}<0,05 \text { PAH vs. Kontrolle/ CTEPH vs. Kontrolle }\end{array}$ \\
\hline \multicolumn{5}{|l}{ Tabelle 18: Vergleich der Leistungsfähigkeit }
\end{tabular}

Im Vergleich der Leistungsfähigkeit zu den unterschiedlichen Belastungszeitpunkten zeigen sich in der frühen Belastungsphase sowie im Bereich der anaeroben Schwelle als auch unter submaximalen Bedingungen signifikante Unterschiede zwischen den Patienten und der gesunden Kontrolle. Die Leistungsfähigkeit der Patienten war im Vergleich zur Kontrollgruppe unter submaximalen Bedingungen um etwa $70 \%$ reduziert, im Bereich der anaeroben Schwelle um ungefähr $65 \%$. 
4.4.2 Sauerstoffaufnahme an den jeweiligen Belastungszeitpunkten

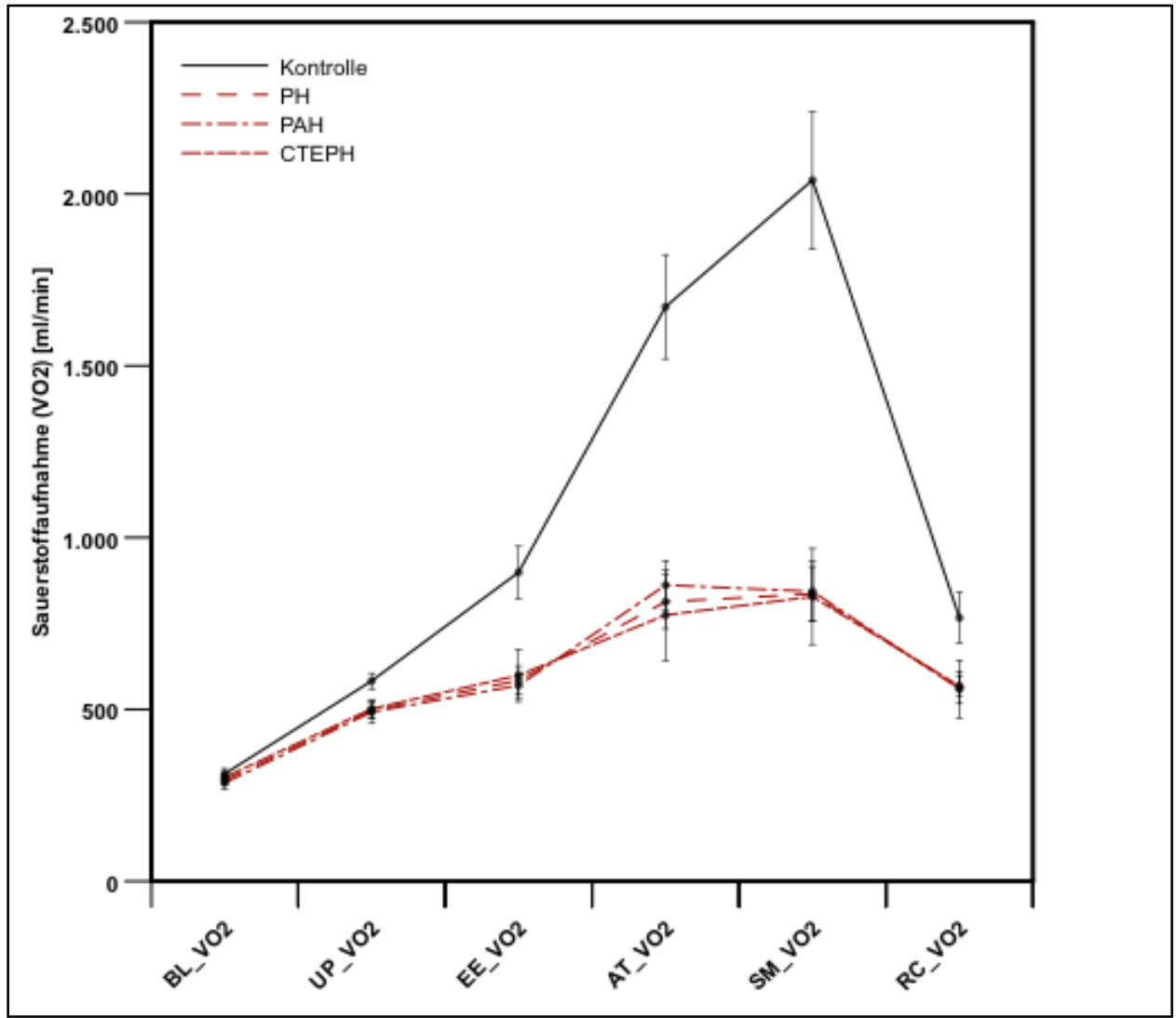

Abbildung 7: Darstellung der Sauerstoffaufnahme zum jeweiligen Belastungszeitpunkt

\begin{tabular}{|l|c|c|c|c|}
\hline $\begin{array}{c}\dot{\mathbf{V}} \mathbf{O}_{2} \\
{[\mathbf{m l} / \mathbf{m i n}]}\end{array}$ & Kontrollgruppe & Patienten & PAH & CTEPH \\
\hline $\mathrm{BL}$ & $311,61 \pm 69,21$ & $293,33 \pm 51,87$ & $285,85 \pm 61,55$ & $302,18 \pm 38,50$ \\
\hline $\mathrm{UP}$ & $582,21 \pm 98,45$ & $496,29 \pm 102,93^{*}$ & $492,00 \pm 118,49 \#$ & $501,36 \pm 86,41(\#)$ \\
\hline $\mathrm{EE}$ & $898,94 \pm 321,74$ & $583,95 \pm 193,03^{*}$ & $569,36 \pm 117,42 \#$ & $598,55 \pm 252,97 \#$ \\
\hline $\mathrm{AT}$ & $1671,83 \pm 643,40$ & $812,83 \pm 330,68^{*}$ & $861,50 \pm 201,38 \#$ & $773,90 \pm 413,79 \#$ \\
\hline $\mathrm{SM}$ & $2040,11 \pm 874,45$ & $835,91 \pm 377,65^{*}$ & $843,25 \pm 302,21 \#$ & $827,91 \pm 461,69 \#$ \\
\hline $\mathrm{RC}$ & $766,83 \pm 309,38$ & $563,08 \pm 227,62^{*}$ & $559,00 \pm 301,37(\#)$ & $567,91 \pm 100,62 \#$ \\
\hline $\begin{array}{l}\text { *p }<0,05 \mathrm{PH} \text { vs. Kontrolle } \\
\# \mathrm{p}<0,05 \text { PAH vs. Kontrolle/ CTEPH vs. Kontrolle, (\#) } \mathrm{p}<0,1 \mathrm{PAH} \text { vs. Kontrolle/ CTEPH vs. Kontrolle }\end{array}$ \\
\hline
\end{tabular}

Tabelle 19: Sauerstoffaufnahme Kontrolle und Patientengruppen

In Bezug auf die Sauerstoffaufnahme zeigen sich zu allen Zeitpunkten der Belastung sowie in der Erholungsphase signifikante Unterschiede zwischen der Kontrollgruppe und dem Patientenkollektiv. Vor allem im Bereich der anaeroben Schwelle $(1671,83 \pm 643,40$ vs. $812,83 \pm 330,68 \mathrm{ml} / \mathrm{min})$ sowie unter submaximaler Belastung $(2040,11 \pm 874,45$ vs. $835,91 \pm 377,65 \mathrm{ml} / \mathrm{min})$ wird der unzureichende Anstieg der Sauerstoffaufnahme der Patienten im Vergleich zur Kontrolle besonders deutlich. 


\subsubsection{Sauerstoffaufnahme pro kg KG}

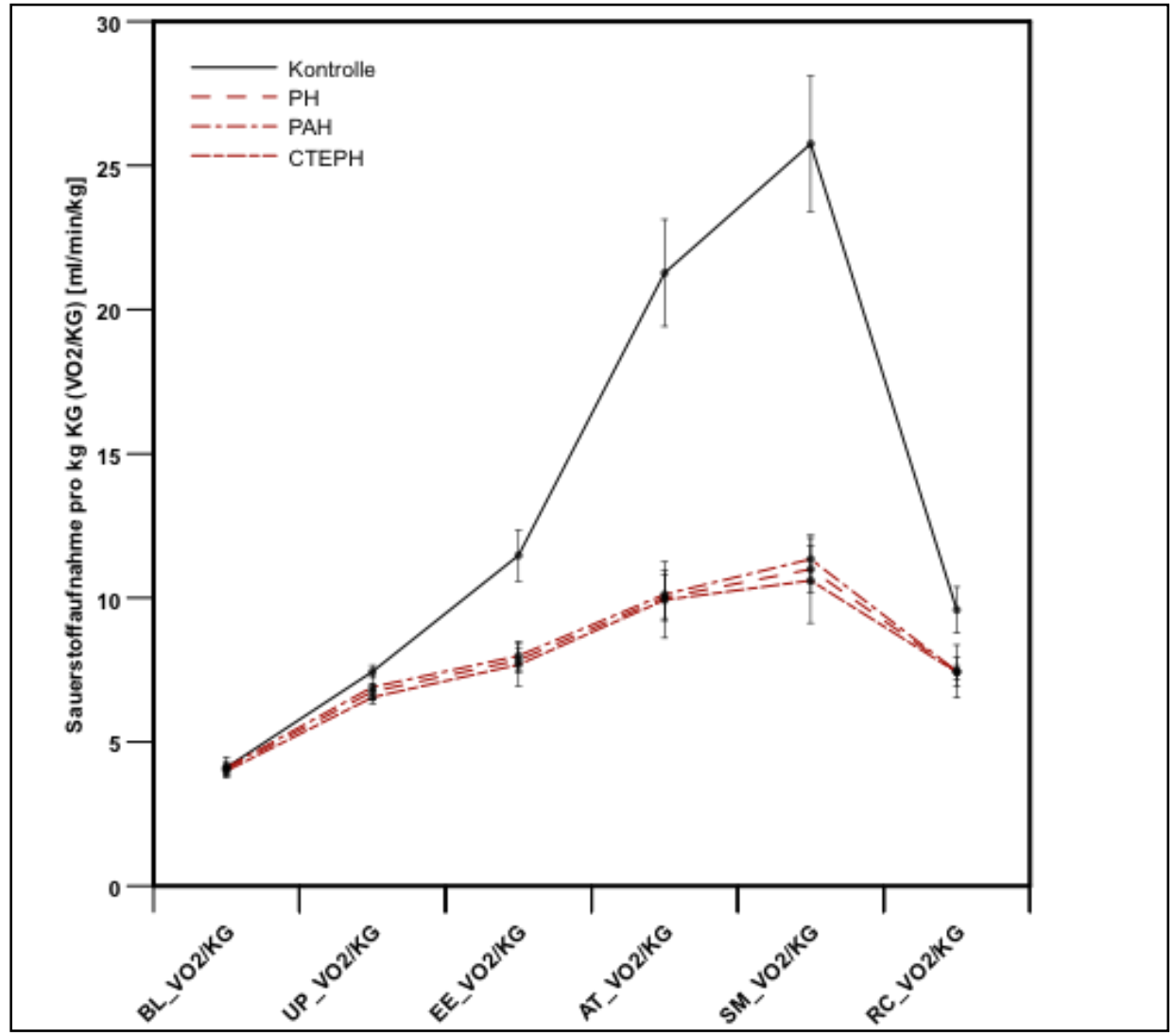

Abbildung 8: Darstellung der Sauerstoffaufnahme pro kg KG

Erfolgt eine Aufschlüsselung der Sauerstoffaufnahme pro kg Körpergewicht, ist auch hier zu allen Zeitpunkten der Untersuchung, ein deutlicher Unterschied zwischen der Patienten- und Kontrollgruppe erkennbar. Das Patientenkollektiv wies auf allen

Belastungsstufen einen unzureichenden Anstieg der Sauerstoffaufnahme im Vergleich zur Kontrollgruppe auf. 


\subsection{4 $\dot{\mathrm{V}} \mathrm{E} / \dot{\mathrm{V}} \mathrm{CO}_{2}$ an den jeweiligen Belastungszeitpunkten}

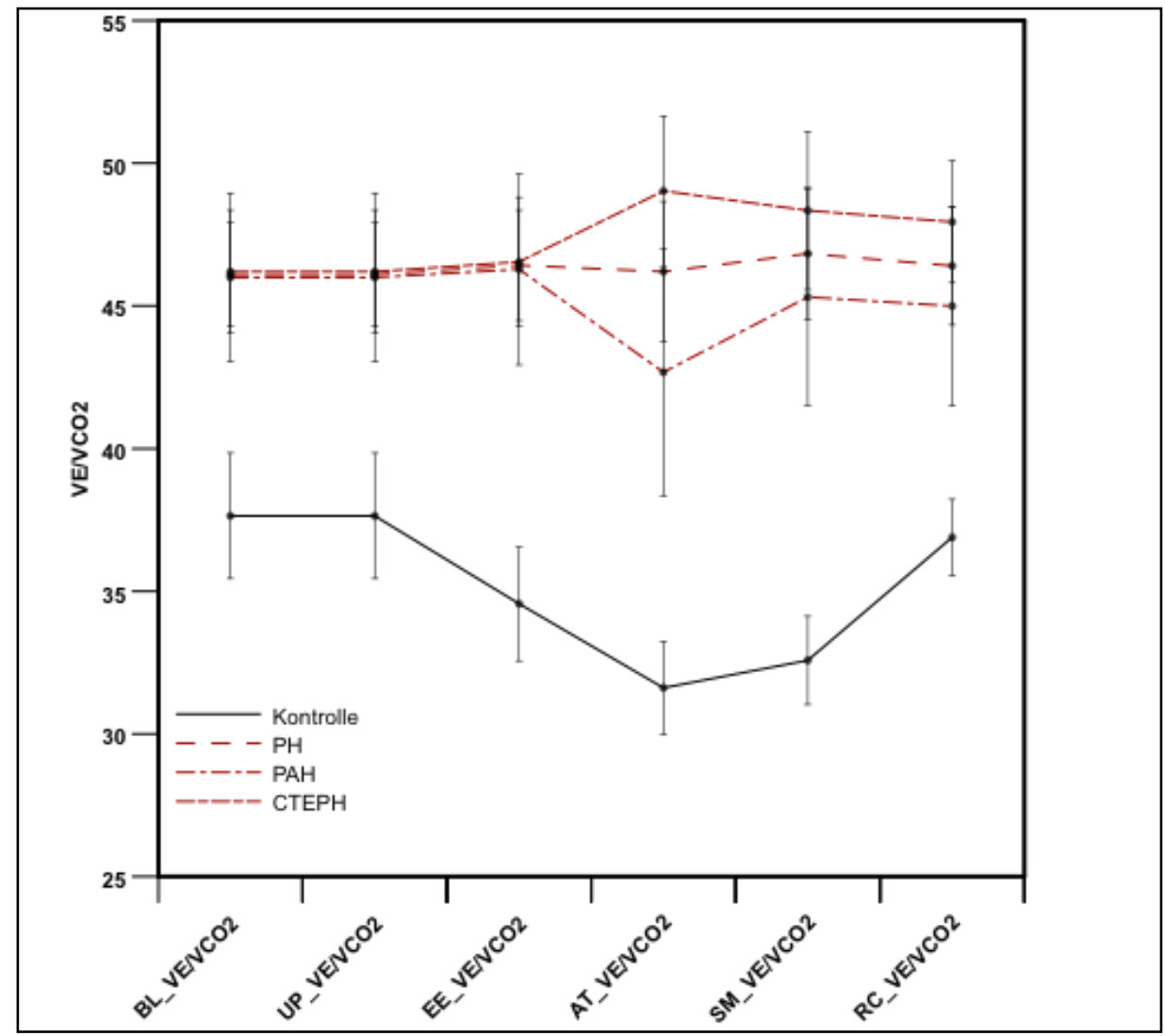

Abbildung 9: Darstellung $\dot{V E}$ VंCO2 an den jeweiligen Belastungszeitpunkten

\begin{tabular}{|c|c|c|c|c|}
\hline$\dot{\mathbf{V E}} /$ & Kontrollgruppe & Patienten & PAH & СТЕРН \\
\hline$\dot{\mathrm{V}} \mathrm{CO}_{2}$ & $\mathrm{MW} \pm \mathrm{SD}$ & $\mathrm{MW} \pm \mathrm{SD}$ & $\mathrm{MW} \pm \mathrm{SD}$ & $\mathrm{MW} \pm \mathrm{SD}$ \\
\hline BL & $39,16 \pm 9,21$ & $46,08 \pm 7,94^{*}$ & $46,00 \pm 10,03 \#$ & $46,16 \pm 5,29 \#$ \\
\hline UP & $37,64 \pm 9,60$ & $46,10 \pm 8,69^{*}$ & $46,00 \pm 10,24 \#$ & $46,20 \pm 7,14 \#$ \\
\hline $\mathrm{EE}$ & $34,56 \pm 8,47$ & $46,42 \pm 8,86^{*}$ & $46,28 \pm 10,58 \#$ & $46,55 \pm 7,51 \#$ \\
\hline AT & $31,61 \pm 6,96$ & $46,21 \pm 10,41 *$ & $42,68 \pm 12,21 \#$ & $49,03 \pm 8,29 \#$ \\
\hline SM & $32,57 \pm 6,74$ & $46,83 \pm 10,87^{*}$ & $45,31 \pm 12,58 \#$ & $48,35 \pm 9,20 \#$ \\
\hline $\mathrm{RC}$ & $36,89 \pm 5,82$ & $46,40 \pm 9,91^{*}$ & $44,99 \pm 12,09(\#)$ & 47,95 $\pm 7,09 \#$ \\
\hline
\end{tabular}

Tabelle 20: $\dot{\mathrm{V}} / \dot{\mathrm{V}} \mathrm{CO}_{2}$ Kontrolle und Patientengruppen

Bei der Darstellung des Atemäquivalentes für $\mathrm{CO} 2\left(\dot{\mathrm{VE}} / \dot{\mathrm{V}} \mathrm{CO}_{2}\right)$ zeigten sich unter Ruhebedingungen als auch $\mathrm{zu}$ jedem Zeitpunkt der Belastung signifikante Unterschiede zwischen der Kontrolle und dem gesamten Patientenkollektiv. Besonders deutlich ist dieser Unterschied im Vergleich der CTEPH-Patienten mit den gesunden Probanden im Bereich der anaeroben Schwelle $(31,61 \pm 6,96$ vs. $49,03 \pm 8,29)$. 


\subsection{Darstellung der mittels kardialer Bioimpedanz ermittelten Parameter}

\subsubsection{Herzindex}

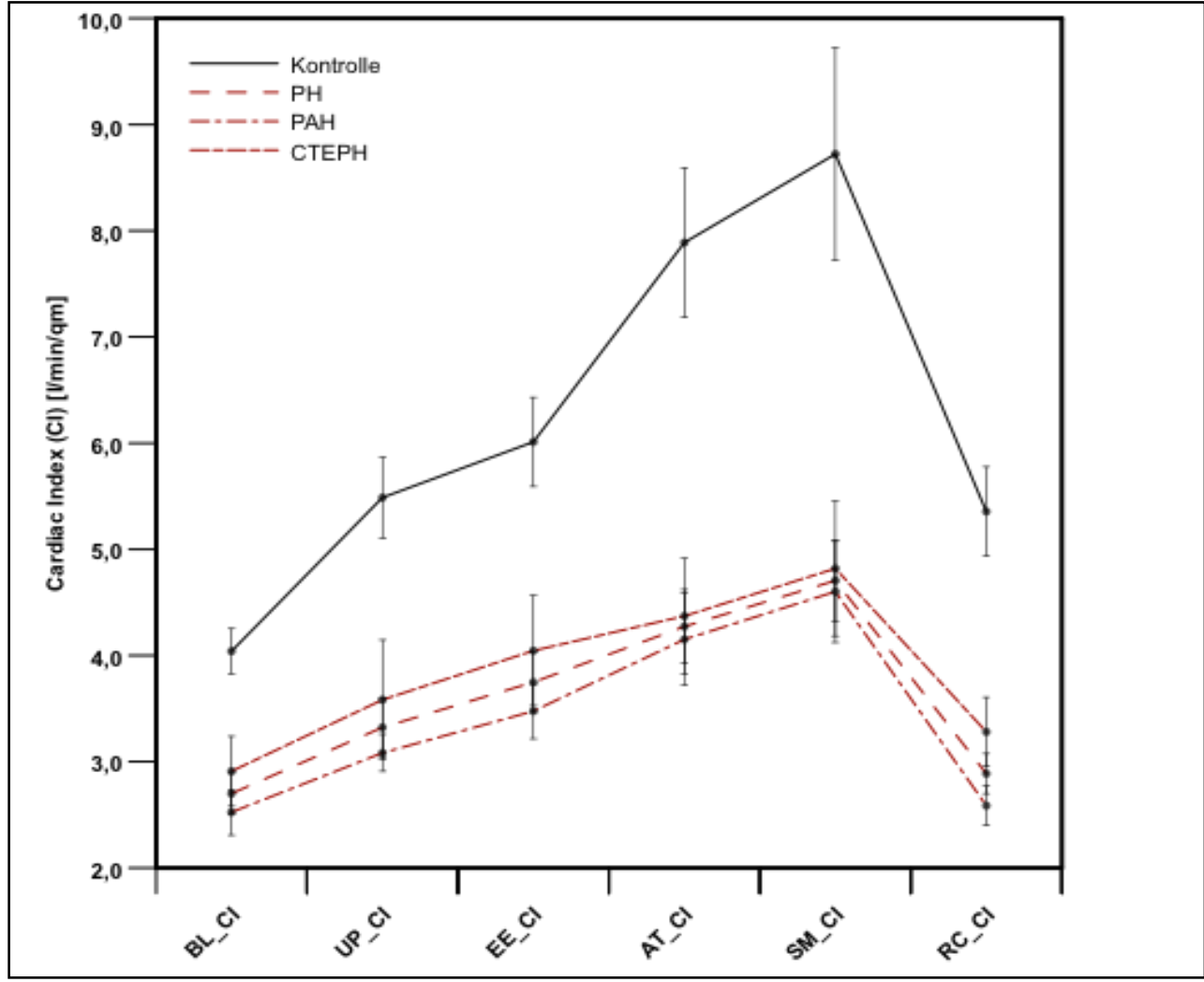

zich des Herzindexes zwischen Patienten- und Kontrollgruppe zu den unterschiedlichen Belastungszeitpunkten

\begin{tabular}{|l|c|c|c|c|}
\hline $\begin{array}{c}\text { CI } \\
{\left[\mathbf{l} / \mathbf{m i n} / \mathbf{m}^{2}\right]}\end{array}$ & Kontrollgruppe & Patienten & PAH & CTEPH \\
\cline { 2 - 5 } & $\mathrm{MW} \pm \mathrm{SD}$ & $\mathrm{MW} \pm \mathrm{SD}$ & $\mathrm{MW} \pm \mathrm{SD}$ & $\mathrm{MW} \pm \mathrm{SD}$ \\
\hline $\mathrm{BL}$ & $4,04 \pm 0,93$ & $2,70 \pm 0,93^{*}$ & $2,52 \pm 0,77 \#$ & $2,91 \pm 1,09 \#$ \\
\hline $\mathrm{UP}$ & $5,49 \pm 1,52$ & $3,32 \pm 1,36^{*}$ & $3,08 \pm 0,60 \#$ & $3,58 \pm 1,87 \#$ \\
\hline $\mathrm{EE}$ & $6,01 \pm 1,83$ & $3,75 \pm 1,35^{*}$ & $3,48 \pm 0,90 \#$ & $4,05 \pm 1,72 \#$ \\
\hline $\mathrm{AT}$ & $7,89 \pm 2,99$ & $4,27 \pm 1,48^{*}$ & $4,15 \pm 1,22 \#$ & $4,37 \pm 1,72 \#$ \\
\hline $\mathrm{SM}$ & $8,72 \pm 4,25$ & $4,70 \pm 1,84^{*}$ & $4,60 \pm 1,65 \#$ & $4,82 \pm 2,11 \#$ \\
\hline $\mathrm{RC}$ & $5,36 \pm 1,79$ & $2,89 \pm 0,90^{*}$ & $2,58 \pm 0,67 \#$ & $3,28 \pm 1,03 \#$ \\
\hline $\begin{array}{l}{ }^{*} \mathrm{p}<0,05 \mathrm{PH} \text { vs. Kontrolle } \\
\# \mathrm{p}<0,05 \mathrm{PAH} \text { vs. Kontrolle/ CTEPH vs. Kontrolle }\end{array}$ \\
\hline
\end{tabular}

Tabelle 21: Herzindex der Patienten- und Kontrollgruppe an den jeweiligen Belastungszeitpunkten

Der ermittelte Herzindex war in der Patientengruppe in Ruhe als auch bei jeder Belastungsstufe gegenüber der Kontrollgruppe deutlich erniedrigt. Unter submaximaler Belastung konnte bei den Patienten im Vergleich zur Kontrolle $\left(4,7 \pm 1,84\right.$ vs. $\left.8,72 \pm 4,251 / \mathrm{min} / \mathrm{m}^{2}\right)$ nur ein unzureichender Anstieg nachgewiesen werden. Gleiches gilt für den Bereich der anaeroben Schwelle $(4,27 \pm 1,48$ vs. 
$7,89 \pm 2,991 / \mathrm{min} / \mathrm{m}^{2}$ ). Auch hier zeigten sich signifikante Unterschiede in Bezug auf die Steigerung des Herzindexes.

\subsubsection{Herzfrequenz}

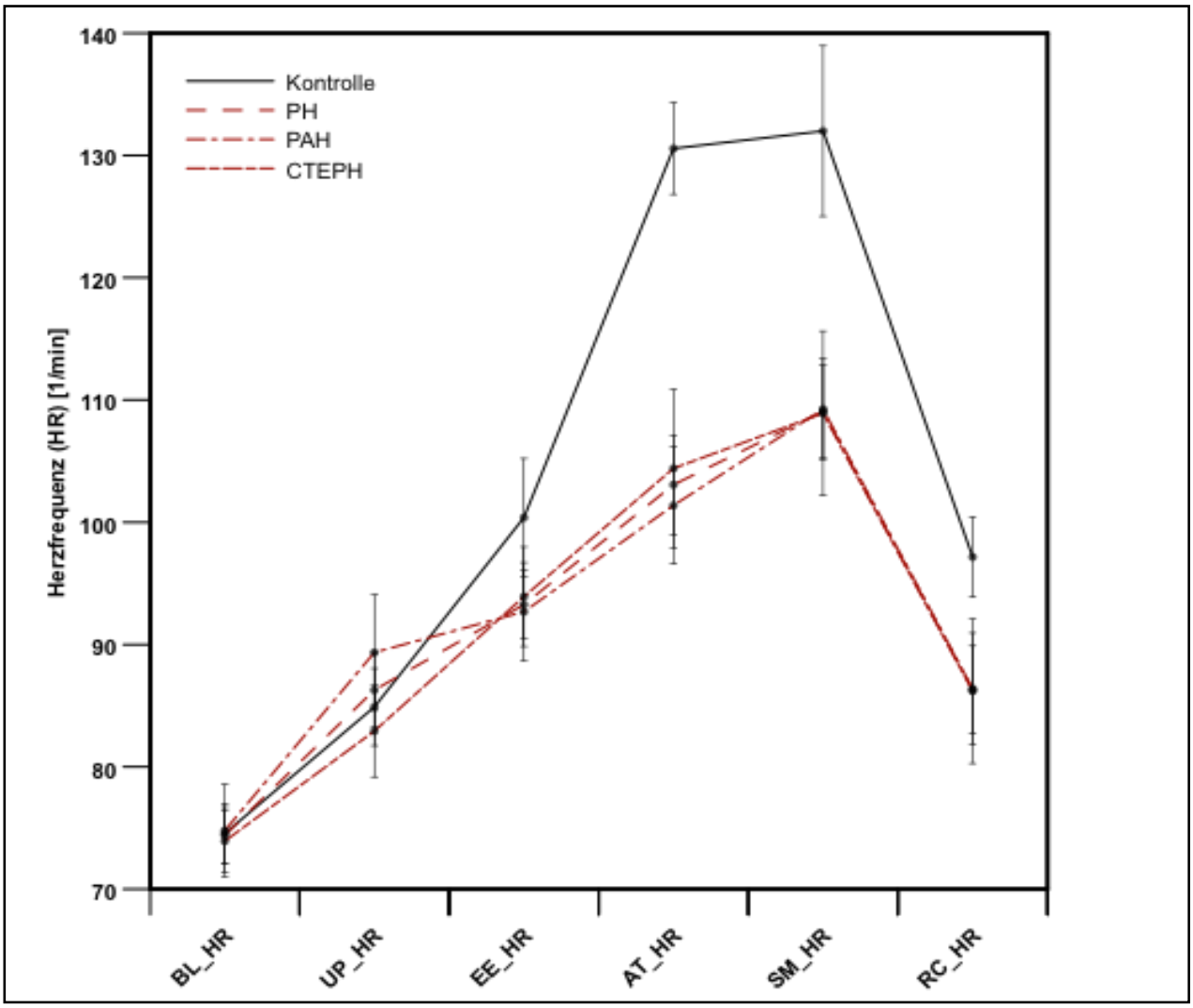

Abbildung 11: $\quad$ Vergleich der Herzfrequenz zwischen Patienten- und Kontrollgruppe zu den unterschiedlichen Belastungszeitpunkten

\begin{tabular}{|l|c|c|c|c|}
\hline $\begin{array}{c}\text { HR } \\
{\left[\mathbf{m i n}^{-1}\right]}\end{array}$ & Kontrollgruppe & Patienten & PAH & CTEPH \\
\hline BL & $74,53 \pm 10,74$ & $74,38 \pm 11,32$ & $74,77 \pm 13,63$ & $73,91 \pm 8,43$ \\
\hline UP & $84,88 \pm 12,65$ & $86,26 \pm 14,65$ & $89,33 \pm 16,34$ & $82,91 \pm 12,44$ \\
\hline EE & $100,39 \pm 20,39$ & $93,26 \pm 13,44$ & $92,67 \pm 13,94$ & $93,91 \pm 13,52$ \\
\hline AT & $130,58 \pm 16,35$ & $103,06 \pm 17,31 *$ & $101,38 \pm 13,50 \#$ & $104,40 \pm 20,48 \#$ \\
\hline SM & $132,00 \pm 29,55$ & $109,09 \pm 18,15^{*}$ & $109,25 \pm 14,30 \#$ & $108,91 \pm 22,35 \#$ \\
\hline RC & $97,16 \pm 14,34$ & $86,29 \pm 17,56^{*}$ & $86,38 \pm 16,44 \#$ & $86,18 \pm 19,61$ \\
\hline $\begin{array}{l}\text { *p }<0,05 \text { PH vs. Kontrolle } \\
\text { \#p }<0,05 \text { PAH vs. Kontrolle/ CTEPH vs. Kontrolle }\end{array}$
\end{tabular}

Tabelle 22: Herzfrequenz der Patienten- und Kontrollgruppe an den jeweiligen Belastungszeitpunkten

Unter Ruhebedingungen $\left(74,53 \pm 10,74\right.$ vs. 74,38 $\left.\pm 11,32 \mathrm{~min}^{-1}\right)$ als auch beim Leertreten $\left(84,88 \pm 12,65\right.$ vs. $\left.86,26 \pm 14,65 \mathrm{~min}^{-1}\right)$ zeigte sich ein nahezu identischer Verlauf der Herzfrequenz. Des Weiteren ist den Daten zu entnehmen, dass das Patientenkollektiv zum Zeitpunkt der submaximalen Belastung $\left(109,09 \pm 18,15 \mathrm{~min}^{-1}\right)$ nicht in der Lage war, im Vergleich zur Kontrollgruppe $\left(132 \pm 29,55 \mathrm{~min}^{-1}\right)$ ihre 
Herzfrequenz adäquat zu steigern. Auch für den Bereich der anaeroben Schwelle $\left(130,58 \pm 16,35\right.$ vs. $\left.103,06 \pm 17,31 \mathrm{~min}^{-1}\right)$ zeigten sich signifikante Unterschiede sowohl zwischen der Kontrolle und dem gesamten Patientenkollektiv als auch im Vergleich der Kontrolle mit den aufgeschlüsselten Patientenklassen.

\subsubsection{Schlagvolumen-Index}

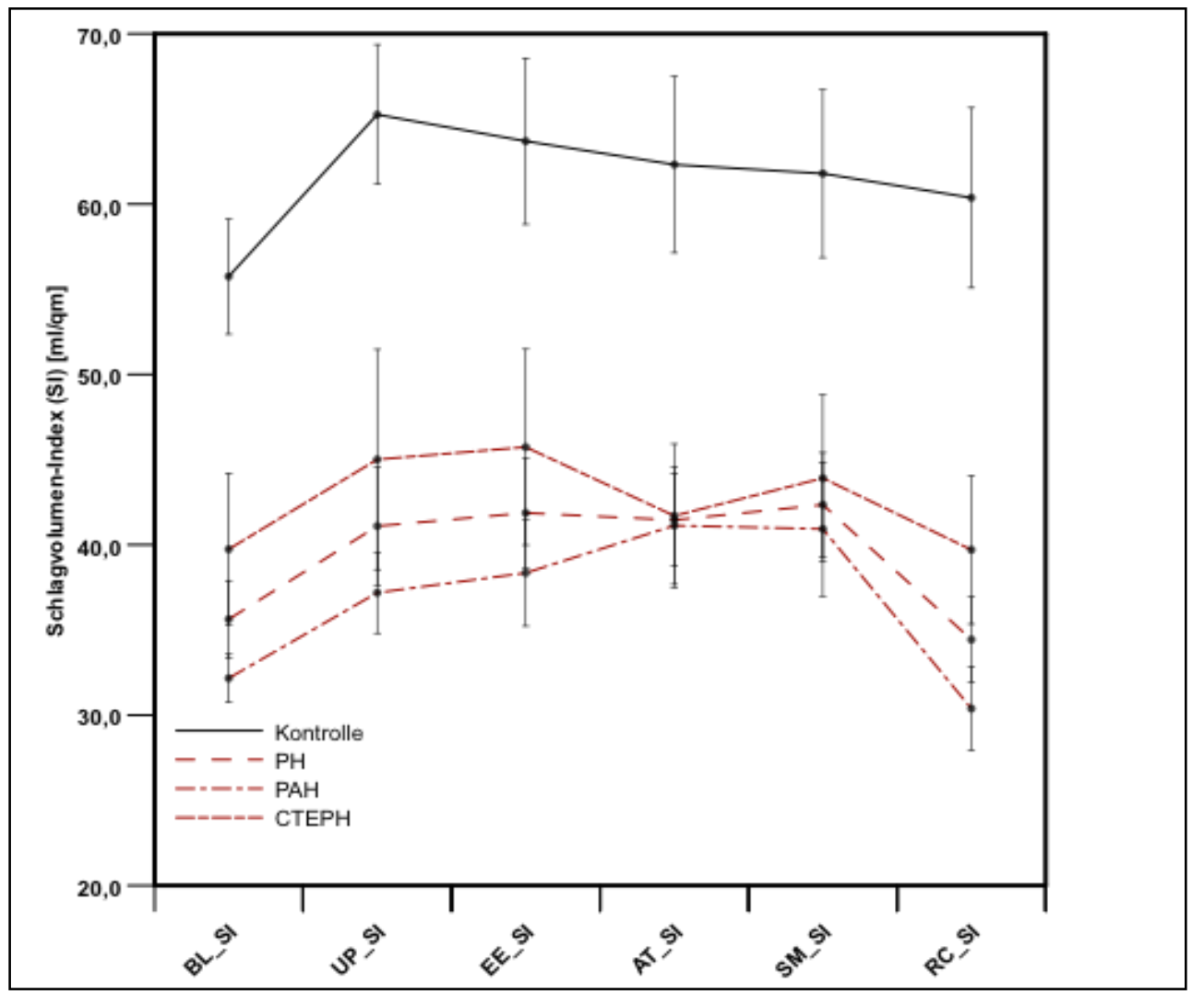

Abbildung 12: $\quad$ Vergleich des Schlagvolumenindexes zwischen Patienten- und Kontrollgruppe zu den

jeweiligen Belastungszeitpunkten

\begin{tabular}{|l|c|l|c|c|}
\hline $\begin{array}{c}\text { SI } \\
{\left[\mathrm{ml} / \mathbf{m}^{2}\right]}\end{array}$ & Kontrollgruppe & Patienten & PAH & CTEPH \\
\hline $\mathrm{BL}$ & $55,74 \pm 14,74$ & $35,63 \pm 11,12^{*}$ & $32,15 \pm 5,10 \#$ & $39,73 \pm 14,81 \#$ \\
\hline $\mathrm{UP}$ & $65,25 \pm 16,41$ & $41,09 \pm 16,24^{*}$ & $37,18 \pm 7,90 \#$ & $45,00 \pm 21,41 \#$ \\
\hline $\mathrm{EE}$ & $63,68 \pm 21,26$ & $41,87 \pm 15,43^{*}$ & $38,33 \pm 10,80 \#$ & $45,73 \pm 19,09 \#$ \\
\hline $\mathrm{AT}$ & $62,32 \pm 22,57$ & $41,44 \pm 11,55^{*}$ & $41,13 \pm 9,75 \#$ & $41,70 \pm 13,34 \#$ \\
\hline $\mathrm{SM}$ & $61,78 \pm 20,87$ & $42,35 \pm 14,65^{*}$ & $40,92 \pm 13,62 \#$ & $43,91 \pm 16,22 \#$ \\
\hline $\mathrm{RC}$ & $60,37 \pm 23,03$ & $34,43 \pm 11,97^{*}$ & $30,38 \pm 8,87 \#$ & $39,70 \pm 13,80 \#(\dagger)$ \\
\hline $\begin{array}{l}\text { *p }<0,05 \text { PH vs. Kontrolle } \\
\# \mathrm{p}<0,05 \text { PAH vs. Kontrolle/ CTEPH vs. Kontrolle } \\
i \mathrm{p}<0,05 \text { PAH v. CTEPH, (†) } \mathrm{p}<0,1 \text { PAH vs. CTEPH }\end{array}$ \\
\hline
\end{tabular}

Tabelle 23: Schlagvolumenindex der Patienten- und Kontrollgruppean den jeweiligen Belastungszeitpunkten

Es zeigte sich bereits unter Ruhebedingungen eine signifikante Reduktion des

Schlagvolumenindexes bei den Patienten $\left(35,63 \pm 11,12 \mathrm{ml} / \mathrm{m}^{2}\right)$ im Vergleich zur

Kontrollgruppe $\left(55,74 \pm 14,74 \mathrm{ml} / \mathrm{m}^{2}\right)$. Der Belastungsverlauf erfolgte annähernd 
parallel, jedoch auf wesentlich niedrigerem Niveau. Im Bereich der Erholungsphase zeigten sich zwischen den beiden Patientengruppen tendenzielle Unterschiede (30,38 $\pm 8,87$ vs. $\left.39,70 \pm 13,80 \mathrm{ml} / \mathrm{m}^{2} ; \mathrm{p}<0,1\right)$ des Schlagvolumenindexes.

\subsubsection{Ejektionsfraktion}

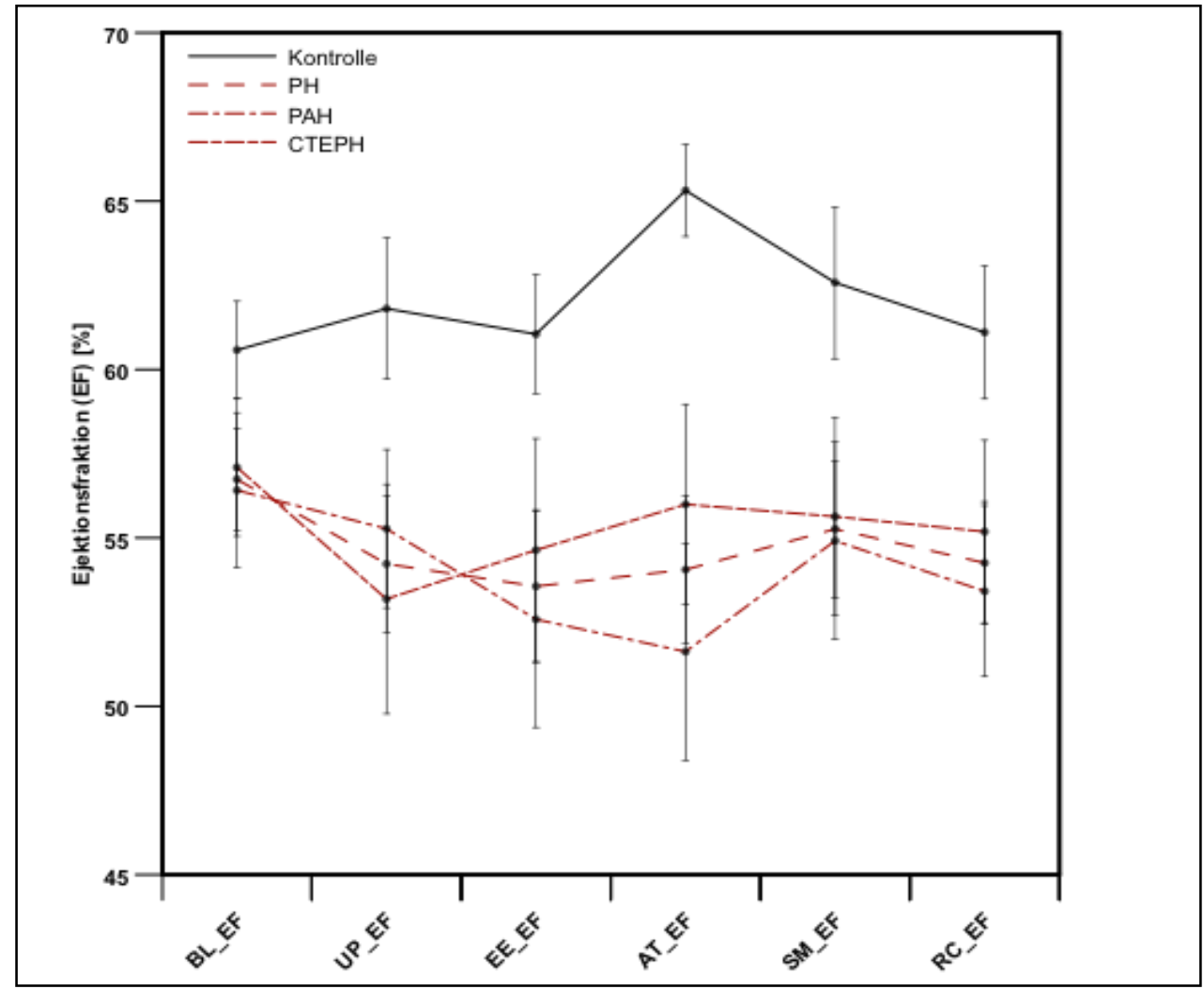

Abbildung 13: $\quad$ Vergleich der Ejektionsfraktion zwischen Patienten- und Kontrollgruppe zu den jeweiligen

Belastungszeitpunkten

\begin{tabular}{|l|c|c|c|c|}
\hline $\begin{array}{c}\text { EF } \\
{[\%]}\end{array}$ & Kontrollgruppe & Patienten & PAH & CTEPH \\
\cline { 2 - 5 } & MW \pm SD & MW \pm SD & MW \pm SD & MW \pm SD \\
\hline BL & $60,58 \pm 6,31$ & $56,74 \pm 7,26\left(^{*}\right)$ & $56,42 \pm 7,97$ & $57,09 \pm 6,76$ \\
\hline UP & $61,81 \pm 8,3$ & $54,23 \pm 9,54^{*}$ & $55,27 \pm 7,81 \#$ & $53,18 \pm 11,30 \#$ \\
\hline EE & $61,06 \pm 7,48$ & $53,57 \pm 10,86^{*}$ & $52,58 \pm 11,11 \#$ & $54,64 \pm 11,00$ \\
\hline AT & $65,32 \pm 5,96$ & $54,06 \pm 9,28^{*}$ & $51,63 \pm 9,12 \#$ & $56,00 \pm 9,40 \#$ \\
\hline SM & $62,58 \pm 9,83$ & $55,26 \pm 9,75^{*}$ & $54,92 \pm 10,13 \#$ & $55,64 \pm 9,79 \#$ \\
\hline RC & $61,11 \pm 8,63$ & $54,26 \pm 8,73^{*}$ & $53,42 \pm 8,80 \#$ & $55,18 \pm 9,00(\#)$ \\
\hline
\end{tabular}

*p $<0,05$ PH vs. Kontrolle, $(*)$ p $<0,1$ PH vs. Kontrolle

\# $\mathrm{p}<0,05$ PAH vs. Kontrolle/ CTEPH vs. Kontrolle, (\#) p $<0,1$ PAH vs. Kontrolle/ CTEPH vs. Kontrolle

Tabelle 24: Ejektionsfraktion der Patienten- und Kontrollgruppe an den jeweiligen Belastungszeitpunkten

Die Ejektionsfraktion setzt sich aus dem Schlagvolumen und dem linksventrikulären

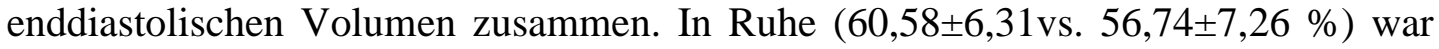
die Ejektionsfraktion in beiden Gruppen vergleichbar. Bereits in der frühen Belastungsphase $(61,06 \pm 7,48$ vs. $53,57 \pm 10,86 \%)$ und auch unter submaximalen 
Bedingungen (62,58 $\pm 9,83$ vs. 55,26 $\pm 9,75 \%)$ fiel der Anstieg in der Kontrollgruppe wesentlich ausgeprägter aus als im Patientenkollektiv. Besonders deutlich zeigt sich dies im direkten Vergleich der Kontrolle mit der Gruppe der PAH-Patienten im Bereich der anaeroben Schwelle $(65,32 \pm 5,96$ vs. $51,63 \pm 9,12 \%)$.

\subsubsection{Enddiastolischer Volumen Index}

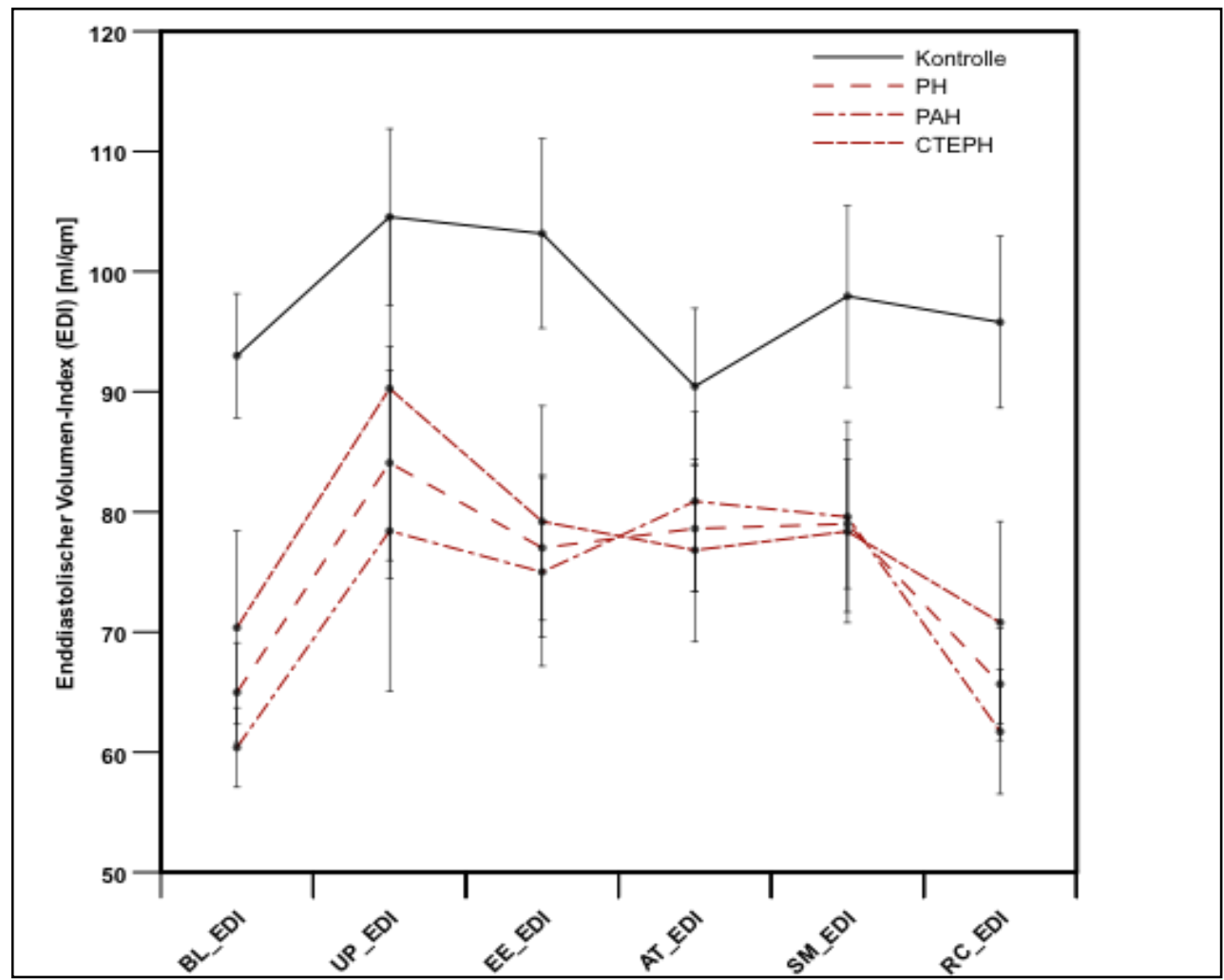

Abbildung 14: $\quad$ Darstellung des enddiastolischen Volumenindexes zwischen Patienten- und Kontrollgruppe zu den jeweiligen Belastungszeitpunkten

\begin{tabular}{|l|c|c|c|c|}
\hline \multirow{2}{*}{$\begin{array}{c}\text { EDI } \\
{\left[\mathbf{m l} / \mathbf{m}^{2}\right]}\end{array}$} & Kontrollgruppe & Patienten & PAH & CTEPH \\
\cline { 2 - 5 } & MW \pm SD & MW \pm SD & MW \pm SD & MW \pm SD \\
\hline BL & $93,00 \pm 22,58$ & $64,96 \pm 20,26^{*}$ & $60,38 \pm 11,93 \#$ & $70,36 \pm 26,71 \#$ \\
\hline UP & $104,56 \pm 29,35$ & $84,09 \pm 46,22^{*}$ & $78,42 \pm 46,37 \#$ & $90,27 \pm 47,47$ \\
\hline EE & $103,17 \pm 33,41$ & $77,00 \pm 28,96^{*}$ & $75,00 \pm 27,18 \#$ & $79,18 \pm 31,97(\#)$ \\
\hline AT & $90,44 \pm 27,53$ & $78,61 \pm 22,26$ & $80,88 \pm 21,12$ & $76,80 \pm 24,11$ \\
\hline SM & $97,95 \pm 32,8$ & $79,00 \pm 25,81^{*}$ & $79,58 \pm 27,48(\#)$ & $78,36 \pm 25,19$ \\
\hline RC & $95,79 \pm 31,15$ & $65,65 \pm 22,39^{*}$ & $61,69 \pm 18,69 \#$ & $70,80 \pm 26,60 \#$ \\
\hline
\end{tabular}

*p $<0,05$ PH vs. Kontrolle

\# p <0,05 PAH vs. Kontrolle/ CTEPH vs. Kontrolle, (\#) p $<0,1 \mathrm{PAH}$ vs. Kontrolle/ CTEPH vs. Kontrolle Tabelle 25: Enddiastolischer Volumenindex der Patienten- und Kontrollgruppe an den jeweiligen Belastungszeitpunkten

Bei der Auswertung des enddiastolischen Volumenindexes (EDI) zeigten sich zu fast jedem Untersuchungszeitpunkt signifikante Unterschiede. Der EDI der Patienten lag in Ruhe $\left(64,96 \pm 20,26\right.$ vs. $\left.93,00 \pm 22,58 \mathrm{ml} / \mathrm{m}^{2}\right)$ sowie unter submaximalen 
Belastungsbedingungen $\left(79,00 \pm 25,81\right.$ vs. $\left.97,95 \pm 32,8 \mathrm{ml} / \mathrm{m}^{2}\right)$ unterhalb der Kontrollgruppe.

\subsubsection{Akzelerationsindex}

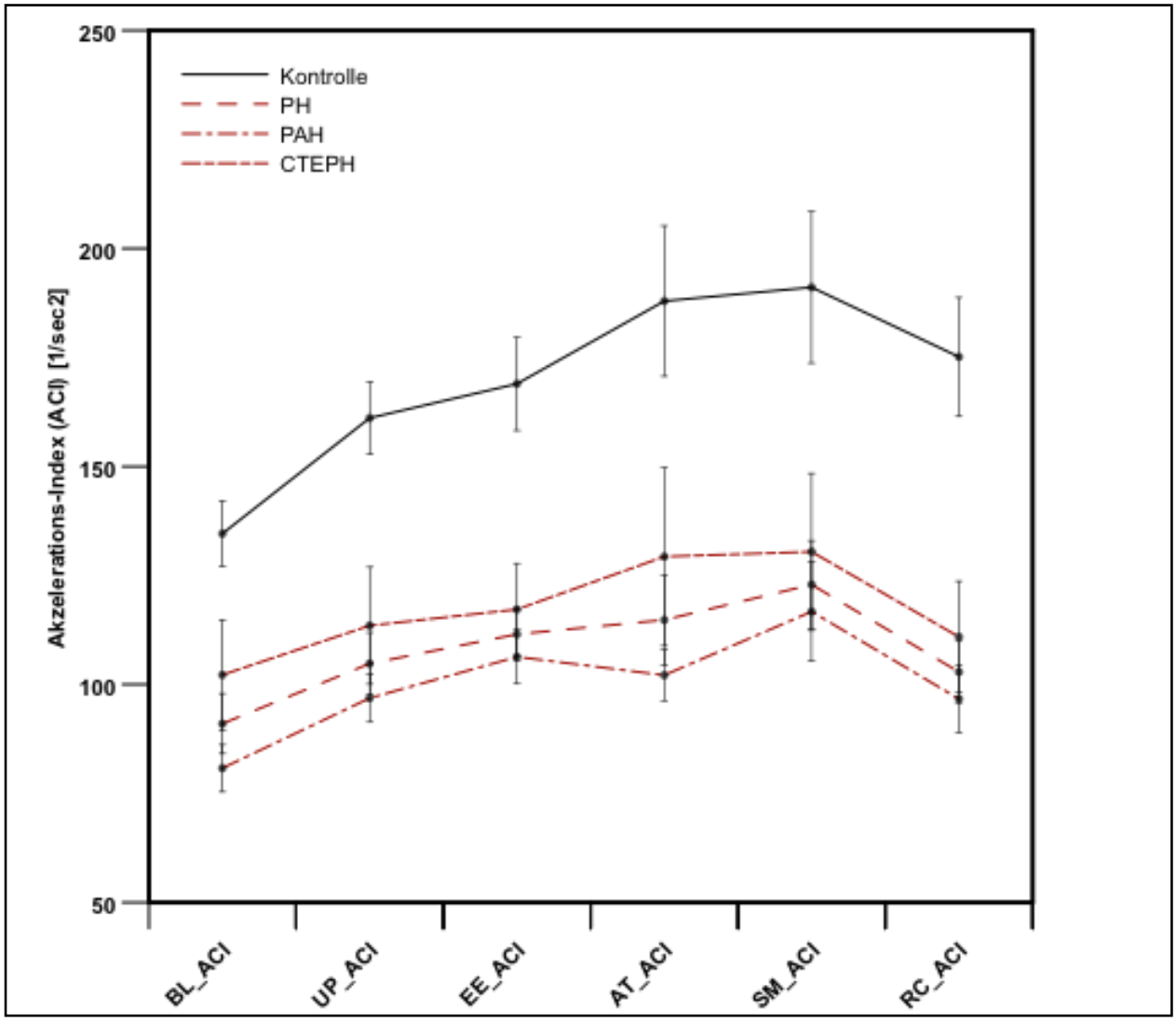

Abbildung 15: $\quad$ Darstellung des Akzelerationsindexes zwischen Patienten- und Kontrollgruppe zu den

jeweiligen Belastungszeitpunkten

\begin{tabular}{|l|c|c|c|c|}
\hline \multirow{2}{*}{$\begin{array}{c}\text { ACI } \\
{\left[\mathbf{s e c}^{-2}\right]}\end{array}$} & Kontrollgruppe & Patienten & PAH & CTEPH \\
\cline { 2 - 5 } & MW \pm SD & MW \pm SD & MW \pm SD & MW \pm SD \\
\hline $\mathrm{BL}$ & $134,58 \pm 32,72$ & $91,00 \pm 33,11^{*}$ & $80,75 \pm 18,76 \#$ & $102,18 \pm 41,97 \#$ \\
\hline $\mathrm{UP}$ & $161,13 \pm 33,38$ & $104,78 \pm 33,97 *$ & $96,83 \pm 19,05 \#$ & $113,45 \pm 44,51 \#$ \\
\hline $\mathrm{EE}$ & $168,95 \pm 46,51$ & $111,52 \pm 28,64 *$ & $106,33 \pm 21,47 \#$ & $117,18 \pm 35,07 \#$ \\
\hline $\mathrm{AT}$ & $187,94 \pm 71,05$ & $114,80 \pm 39,80^{*}$ & $102,13 \pm 16,47 \#$ & $129,29 \pm 54,04 \#$ \\
\hline $\mathrm{SM}$ & $191,07 \pm 65,05$ & $122,90 \pm 45,07 *$ & $116,73 \pm 37,90 \#$ & $130,44 \pm 53,97 \#$ \\
\hline $\mathrm{RC}$ & $175,11 \pm 57,90$ & $102,87 \pm 33,82^{*}$ & $96,69 \pm 27,75 \#$ & $110,90 \pm 40,53 \#$ \\
\hline $\begin{array}{l}* \mathrm{p}<0,05 \mathrm{PH} \text { vs. Kontrolle } \\
\# \mathrm{p}<0,05 \mathrm{PAH} \text { vs. Kontrolle/ CTEPH vs. Kontrolle }\end{array}$ \\
\hline
\end{tabular}

Tabelle 26: Akzelerationsindex der Patienten- und Kontrollgruppe an den jeweiligen Belastungszeitpunkten

Der Akzelerationsindex repräsentiert einen Parameter der vorlastunabhängigen Inotropie. In der graphischen Darstellung ist zu erkennen, dass die Patienten nicht in der Lage waren, im Vergleich zur Kontrolle ihre Inotropie im Belastungsverlauf signifikant zu steigern. Besonders deutlich wird dieser Sachverhalt zum Zeitpunkt der submaximalen Belastung $\left(191,07 \pm 65,05\right.$ vs. $122,90 \pm 45,07 \mathrm{sec}^{-2}$ und an der 
anaeroben Schwelle $\left(187,94 \pm 71,05 \quad\right.$ vs. $\left.114,80 \pm 39,80 \quad \mathrm{sec}^{-2}\right)$. Bei der Gegenüberstellung der beiden Patientengruppen in Relation $\mathrm{zu}$ den gesunden Probanden zeigten sich zu jedem Untersuchungszeitpunkt signifikante Unterschiede.

\subsubsection{Peak Flow Index}

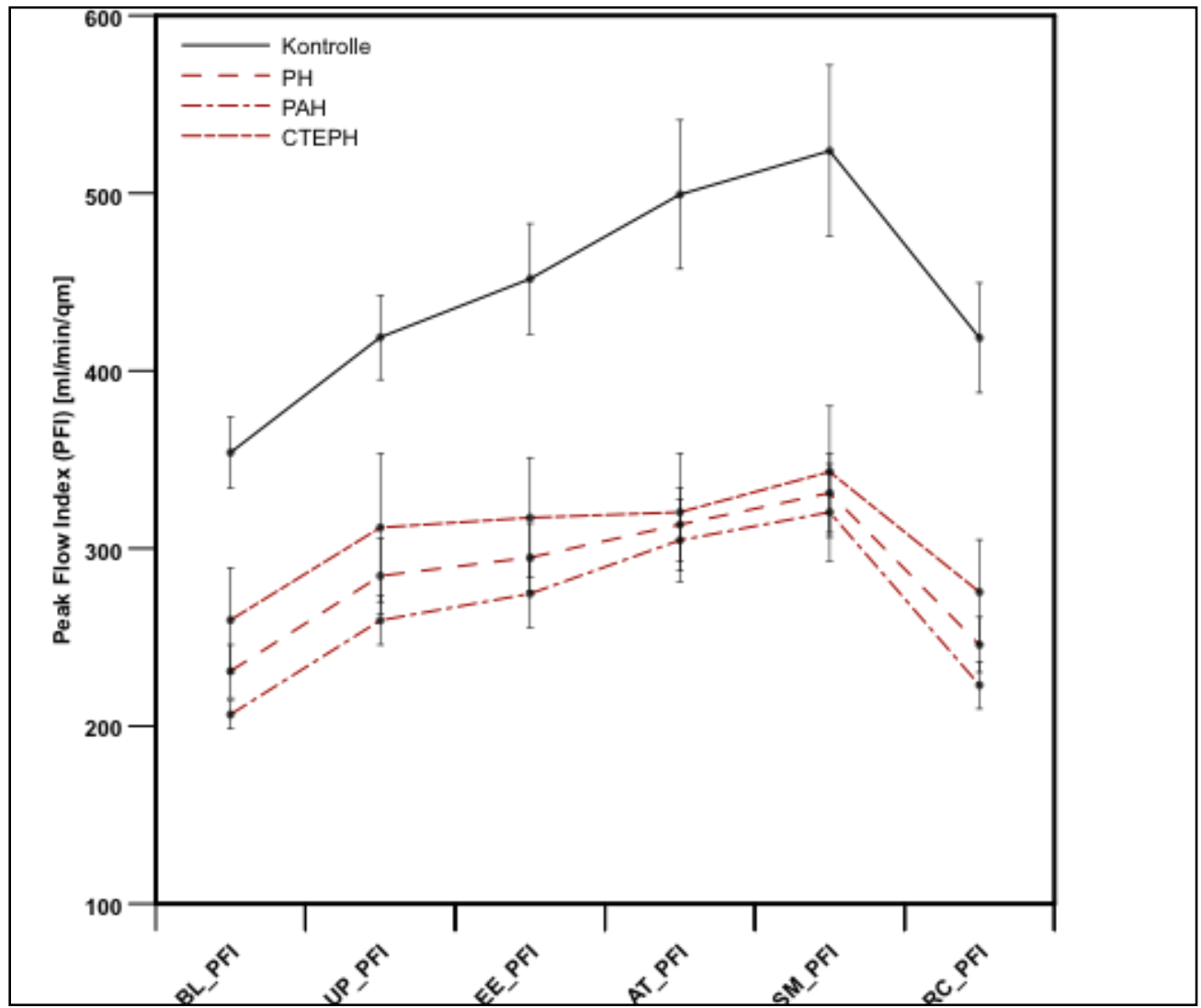

Abbildung 16: $\quad$ Darstellung des Peak Flow Indexes zwischen Patienten- und Kontrollgruppe zu den jeweiligen Belastungszeitpunkten

\begin{tabular}{|l|c|c|c|c|}
\hline $\begin{array}{c}\text { PFI } \\
{[\mathbf{m l} / \mathbf{s e c} /} \\
\left.\mathbf{m}^{2}\right]\end{array}$ & Kontrollgruppe & Patienten & PAH & CTEPH \\
\hline BL & $353,84 \pm 87,65$ & $230,79 \pm 73,14^{*}$ & $206,46 \pm 29,38 \#$ & MW \pm SD \\
\hline UP & $418,75 \pm 95,01$ & $284,39 \pm 102,81^{*}$ & $259,42 \pm 47,65 \#$ & $311,64 \pm 138,52 \#$ \\
\hline EE & $451,68 \pm 135,53$ & $294,87 \pm 91,57 *$ & $274,42 \pm 66,11 \#$ & $317,18 \pm 112,21 \#$ \\
\hline AT & $499,16 \pm 182,83$ & $313,33 \pm 87,29^{*}$ & $304,50 \pm 65,22 \#$ & $320,40 \pm 104,68 \#$ \\
\hline SM & $523,74 \pm 209,92$ & $331,17 \pm 106,83^{*}$ & $320,25 \pm 94,62 \#$ & $343,09 \pm 122,32 \#$ \\
\hline RC & $418,47 \pm 134,55$ & $245,74 \pm 74,32^{*}$ & $223,00 \pm 47,84 \#$ & $275,30 \pm 93,44 \#$ \\
\hline $\begin{array}{l}* \\
\text { \# }<0,05 \text { PH vs. Kontrolle }\end{array}$ & MW \pm SD & & \\
\hline
\end{tabular}

Tabelle 27: Peak Flow Index der Patienten- und Kontrollgruppe an den jeweiligen Belastungszeitpunkten

Aus dem Unvermögen der Patienten ihre Inotropie im Belastungsverlauf zu steigern, resultiert ein reduzierter Peak Flow Index zum Zeitpunkt des Auswurfes in allen Belastungsstufen sowie unter Ruhebedingungen $(353,84 \pm 87,65$ vs. 
$\left.230,79 \pm 73,14 \mathrm{ml} / \mathrm{sec} / \mathrm{m}^{2}\right)$. Besonders deutlich zeigt sich dieser Unterschied im direkten Vergleich der Kontrolle mit der Gruppe der PAH-Patienten beispielsweise

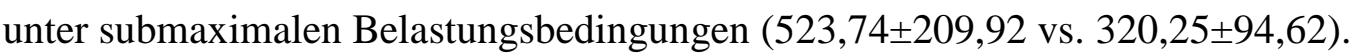

\subsubsection{Ejektionsverhältnis}

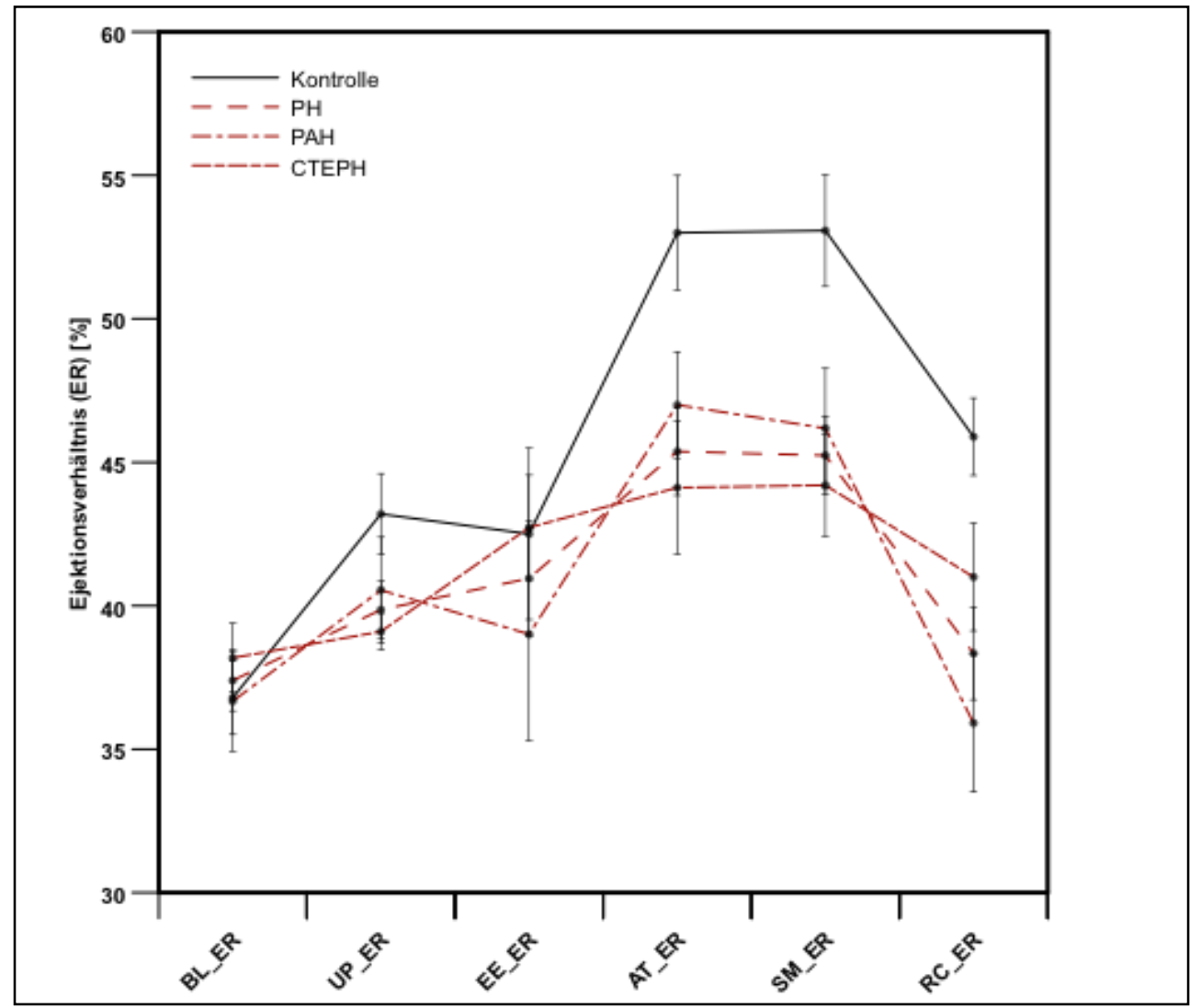

Abbildung 17: $\quad$ Darstellung des Ejektionsverhältnisses zwischen Patienten- und Kontrollgruppe zu den jeweiligen Belastungszeitpunkten

\begin{tabular}{|l|c|c|c|c|}
\hline $\begin{array}{c}\text { ER } \\
{[\%]}\end{array}$ & Kontrollgruppe & Patienten & PAH & CTEPH \\
\hline BL & MW \pm SD & MW \pm SD & MW \pm SD & MW \pm SD \\
\hline UP & $36,79 \pm 5,59$ & $37,39 \pm 5,09$ & $36,67 \pm 6,01$ & $38,18 \pm 4,00$ \\
\hline EE & $43,20 \pm 5,44$ & $39,86 \pm 4,59\left(^{*}\right)$ & $40,55 \pm 6,11$ & $39,10 \pm 2,02(\#)$ \\
\hline AT & $53,00 \pm 7,46$ & $45,38 \pm 6,12^{*}$ & $47,00 \pm 4,90 \#$ & $44,11 \pm 6,94 \#$ \\
\hline SM & $53,08 \pm 7,04$ & $45,24 \pm 6,28^{*}$ & $46,18 \pm 6,94 \#$ & $44,20 \pm 5,65 \#$ \\
\hline RC & $45,89 \pm 5,74$ & $38,33 \pm 7,38$ & $35,91 \pm 7,98 \#$ & $41,00 \pm 5,94(\#)(\dagger)$ \\
\hline $\begin{array}{l}* \text { p }<0,05 \text { PH vs. Kontrolle, },(*) \text { p }<0,1 \text { PH vs. Kontrolle } \\
\# \text { p }<0,05 \text { PAH vs. Kontrolle/ CTEPH vs. Kontrolle, (\#) }<<0,1 \text { PAH vs. Kontrolle/ CTEPH vs. Kontrolle } \\
t \mathrm{p}<0,05 \text { PAH vs. CTEPH, (†) }<<0,1 \text { PAH vs. CTEPH }\end{array}$
\end{tabular}
Tabelle 28: $\quad$ Ejektionsverhältnis der Patienten- und Kontrollgruppe an den jeweiligen

Belastungszeitpunkten

Das Ejektionsverhältnis (ER) setzt sich aus der ventrikulären Ejektionszeit (VET) sowie dem RR-Intervall zusammen. Bei Gesunden sinkt bei ansteigender Herzfrequenz und Belastung die Ejektionszeit zu Lasten der Diastolendauer. Diese 
Gegebenheit war in der Patientengruppe deutlich geringer ausgeprägt als bei der Kontrolle. Des Weiteren konnte bei den Patienten eine veränderte VET beobachtet werden. Dies führte dazu, dass das Patientenkollektiv unter körperlicher Belastung eine verlängerte Diastole aufwies.

\subsubsection{Kontraktilitätsindex}

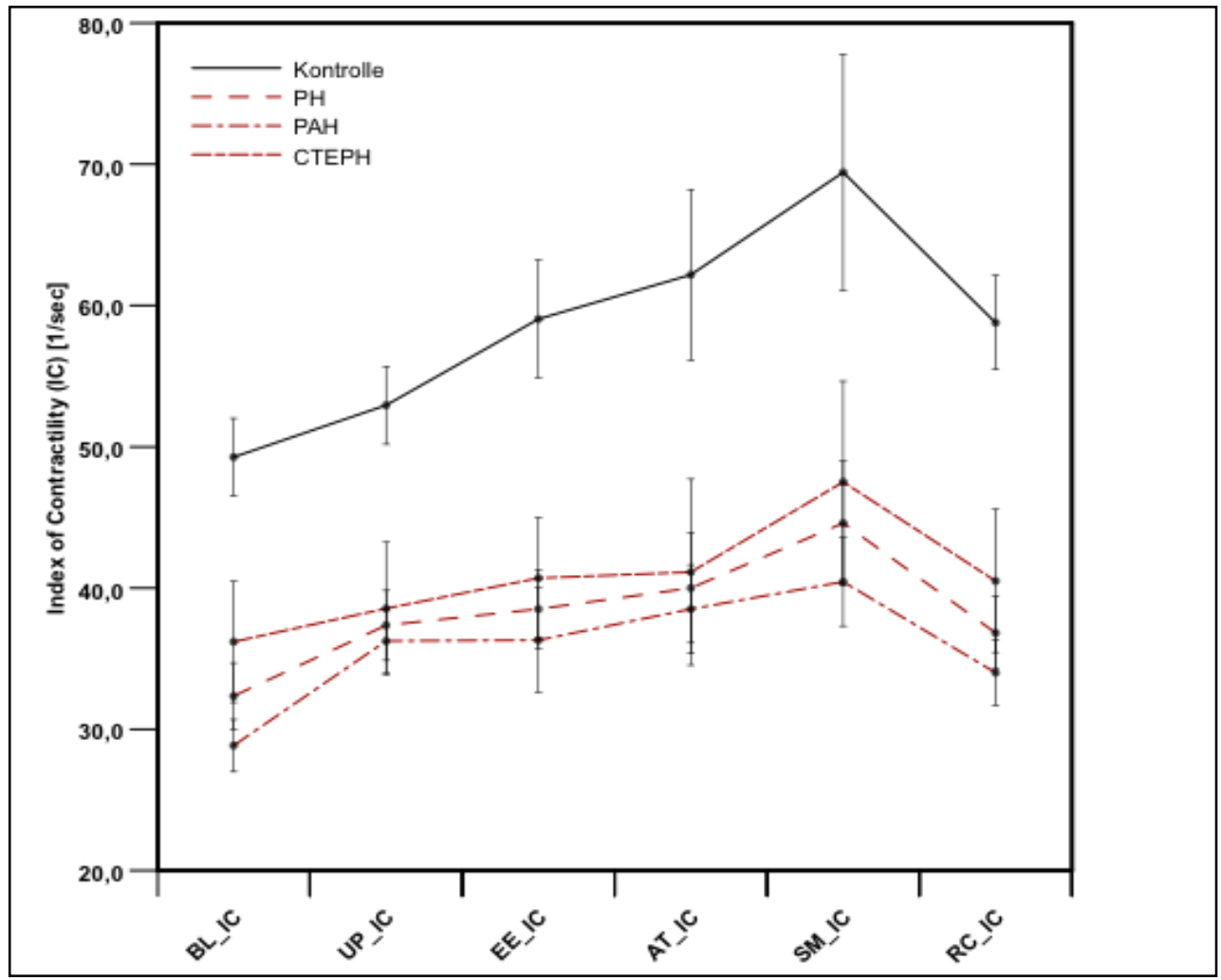

Abbildung 18: Darstellung des Kontraktilitätsindexes

\begin{tabular}{|l|c|c|c|c|}
\hline $\begin{array}{c}\text { IC } \\
{[\mathbf{1} / \mathbf{s e c}]}\end{array}$ & Kontrollgruppe & Patienten & PAH & CTEPH \\
\hline BL & MW \pm SD & MW \pm SD & MW \pm SD & MW \pm SD \\
\hline UP & $49,26 \pm 11,99$ & $32,35 \pm 11,21^{*}$ & $28,83 \pm 6,38 \#$ & $36,18 \pm 14,17 \#$ \\
\hline EE & $52,93 \pm 10,67$ & $37,35 \pm 11,92^{*}$ & $36,25 \pm 7,79 \#$ & $38,55 \pm 15,58 \#$ \\
\hline AT & $59,05 \pm 18,16$ & $38,50 \pm 12,48^{*}$ & $36,30 \pm 11,70 \#$ & $40,70 \pm 13,47 \#$ \\
\hline SM & $62,18 \pm 24,93$ & $40,00 \pm 14,53^{*}$ & $38,50 \pm 7,69 \#$ & $41,13 \pm 18,62 \#$ \\
\hline RC & $58,79 \pm 14,50$ & $36,83 \pm 12,35^{*}$ & $34,00 \pm 8,28 \#$ & $40,50 \pm 15,97 \#$ \\
\hline $\begin{array}{l}\text { *p }<0,05 \text { PH vs. Kontrolle } \\
\# \mathrm{p}<0,05 \text { PAH vs. Kontrolle/ CTEPH vs. Kontrolle, (\#) p }<0,1 \text { PAH vs. Kontrolle/ CTEPH vs. Kontrolle }\end{array}$ \\
\hline
\end{tabular}

Tabelle 29: Kontraktilitätsindex zwischen Kontrolle und Patientengruppen

Der Kontraktilitätsindex (IC) ist neben dem Peak Flow ein volumenabhängiger

Kontraktilitätsparameter. Sowie unter Ruhebedingungen als auch unter Belastung zeigen sich signifikante Unterschiede zwischen dem gesamten Patientenkollektiv im Vergleich zur gesunden Kontrolle als auch zwischen der Kontrolle und den einzelnen 
Patientenklassen. Vor allem unter submaximalen Bedingungen waren die PAH-Patienten nicht in der Lage, ihre Kontraktilität im Vergleich zur Kontrollgruppe adäquat zu steigern $(69,43 \pm 31,28$ vs. $40,43 \pm 8,281 / \mathrm{sec})$.

\subsubsection{Thorakaler Flüssigkeitsindex}

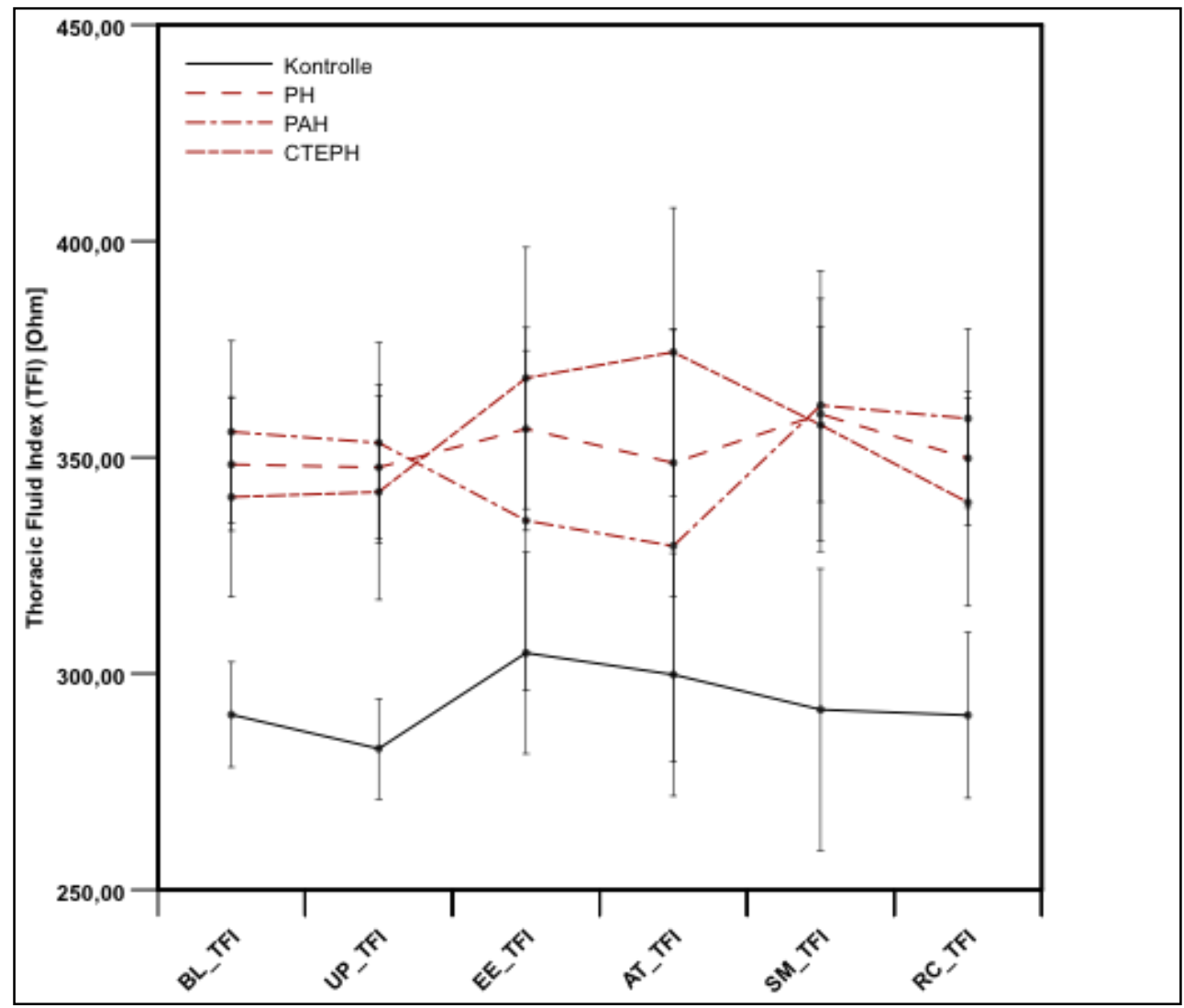

Abbildung 19: Darstellung des thorakalen Flüssigkeitsindexes

\begin{tabular}{|c|c|c|c|c|}
\hline \multirow{2}{*}{$\begin{array}{c}\text { TFI } \\
{[\mathrm{Ohm}]}\end{array}$} & Kontrollgruppe & Patienten & PAH & СТЕРН \\
\hline & $\mathrm{MW} \pm \mathrm{SD}$ & $\mathrm{MW} \pm \mathrm{SD}$ & $\mathrm{MW} \pm \mathrm{SD}$ & $\mathrm{MW} \pm \mathrm{SD}$ \\
\hline $\mathrm{BL}$ & $290,50 \pm 48,53$ & $348,36 \pm 71,87 *$ & $355,91 \pm 69,64 \#$ & $340,82 \pm 76,63(\#)$ \\
\hline UP & $282,60 \pm 44,93$ & $347,70 \pm 74,25 *$ & $353,40 \pm 73,22 \#$ & $342,00 \pm 78,77(\#)$ \\
\hline $\mathrm{EE}$ & $304,73 \pm 90,14$ & $356,57 \pm 87,83$ & $335,40 \pm 87,86$ & $368,33 \pm 90,78$ \\
\hline $\mathrm{AT}$ & $299,77 \pm 101,21$ & $348,71 \pm 81,75$ & $329,50 \pm 99,94$ & $374,33 \pm 57,84$ \\
\hline SM & $291,67 \pm 97,73$ & $360,00 \pm 60,98(*)$ & $362,00 \pm 69,74$ & $357,50 \pm 58,46$ \\
\hline $\mathrm{RC}$ & $290,33 \pm 74,24$ & $349,76 \pm 71,13^{*}$ & $359,00 \pm 68,88 \#$ & $339,60 \pm 75,85(\#)$ \\
\hline
\end{tabular}

Tabelle 30: Thorakaler Flüssigkeitsindex zwischen Kontrolle und Patientengruppen

Der thorakale Flüssigkeitsindex (TFI) spiegelt die Gesamtimpedanz des Thorax wieder. Mit steigendem Flüssigkeitsgehalt kommt es $\mathrm{zu}$ einer Zunahme der Leitfähigkeit. Dies spiegelt sich in der Abnahme des TFI wieder. Signifikante Unterschiede zeigten sich im Vergleich der Kontrolle mit dem gesamten 
Patientenkollektiv besonders unter Ruhebedingungen (290,50 $\pm 48,53$ vs. $348,36 \pm 71,87 \mathrm{Ohm})$ als auch im Bereich der Erholungsphase $(290,33 \pm 74,24$ vs. $349,76 \pm 71,13 \mathrm{Ohm})$.

\subsubsection{Ventrikuläre Ejektionszeit}

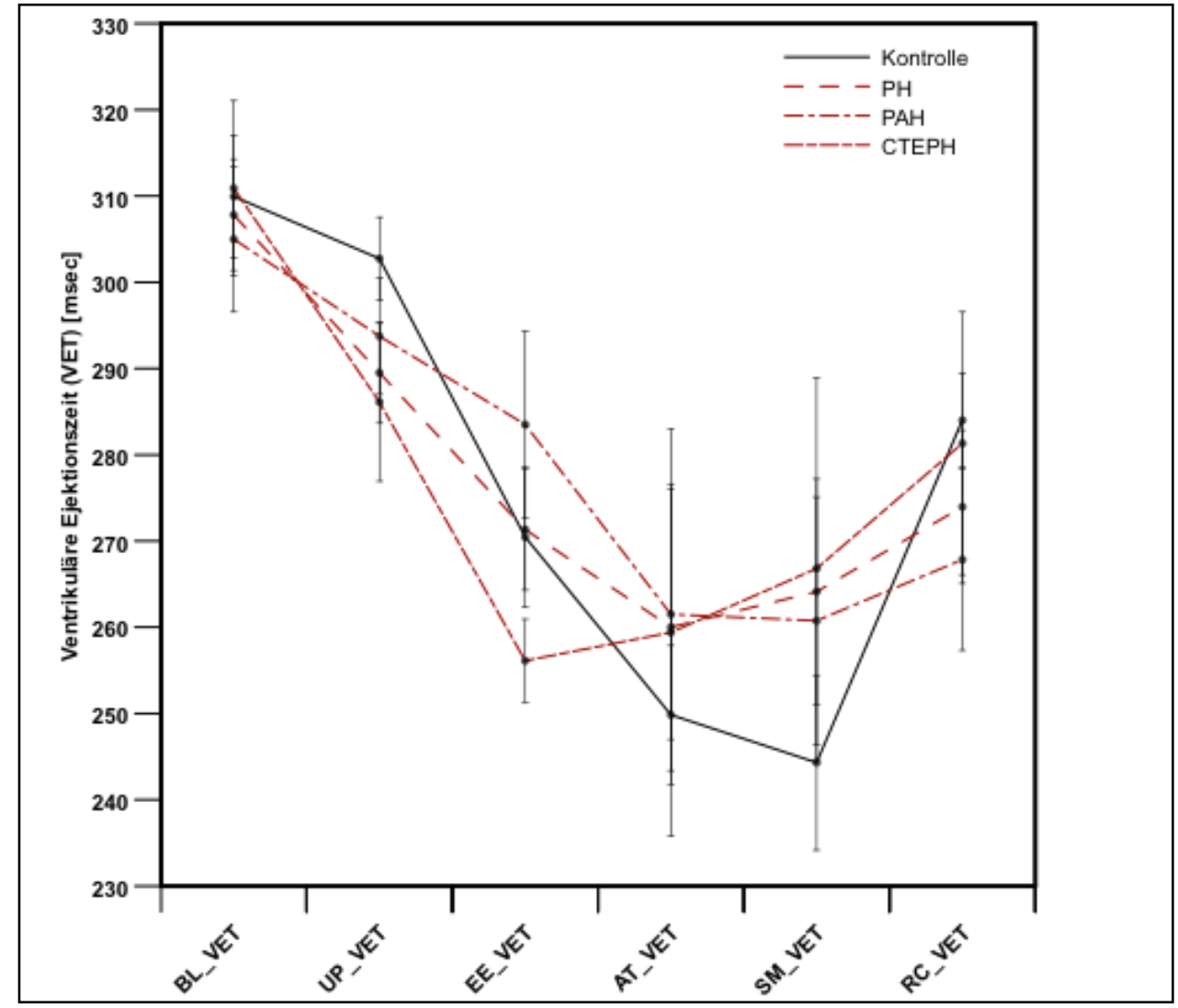

Abbildung 20: Darstellung der ventrikulären Ejektionszeit

\begin{tabular}{|c|c|c|c|c|}
\hline \multirow{2}{*}{$\begin{array}{c}\text { VET } \\
{[\mathrm{msec}]}\end{array}$} & Kontrollgruppe & Patienten & $\overline{\text { PAH }}$ & СТЕРН \\
\hline & $\mathrm{MW} \pm \mathrm{SD}$ & $\mathrm{MW} \pm \mathrm{SD}$ & $\mathrm{MW} \pm \mathrm{SD}$ & $\mathrm{MW} \pm \mathrm{SD}$ \\
\hline $\mathrm{BL}$ & $309,95 \pm 31,00$ & $307,83 \pm 30,85$ & $305,00 \pm 29,29$ & $310,91 \pm 33,63$ \\
\hline UP & $302,75 \pm 19,21$ & $289,50 \pm 24,70\left(^{*}\right)$ & $293,75 \pm 19,05$ & $286,10 \pm 29,00(\#)$ \\
\hline $\mathrm{EE}$ & $270,47 \pm 33,73$ & $271,33 \pm 29,84$ & $283,50 \pm 34,18$ & $256,13 \pm 13,59 \dagger$ \\
\hline AT & $249,85 \pm 29,00$ & $260,00 \pm 43,95$ & $261,50 \pm 20,51$ & $259,40 \pm 52,83$ \\
\hline SM & $244,30 \pm 32,11$ & $264,11 \pm 39,27$ & $260,75 \pm 28,72$ & $266,80 \pm 49,45$ \\
\hline $\mathrm{RC}$ & $284,00 \pm 23,26$ & $273,95 \pm 41,66$ & $267,83 \pm 36,37$ & $281,30 \pm 48,21$ \\
\hline
\end{tabular}

Tabelle 31: Ventrikuläre Ejektionszeit zwischen Kontrolle und Patientengruppen

Die ventrikuläre Ejektionszeit (VET) ist u.a. von der Ejektionsfraktion und der Herzfrequenz abhängig. Lediglich in der frühen Belastungsphase zeigt sich ein tendenziell signifikanter Unterschied der VET im direkten Vergleich der Patienten mit pulmonal arterieller Hypertonie und chronisch-thromboembolischer pulmonaler 
Hypertonie $(283,50 \pm 34,18$ vs. $256,13 \pm 13,59 \mathrm{msec})$. Bei der Gegenüberstellung des

Patientenkollektives mit der Kontrolle zeigten sich zu keinem Zeitpunkt der Belastung wesentliche Unterschiede.

\subsubsection{Systolisches Zeitverhältnis}

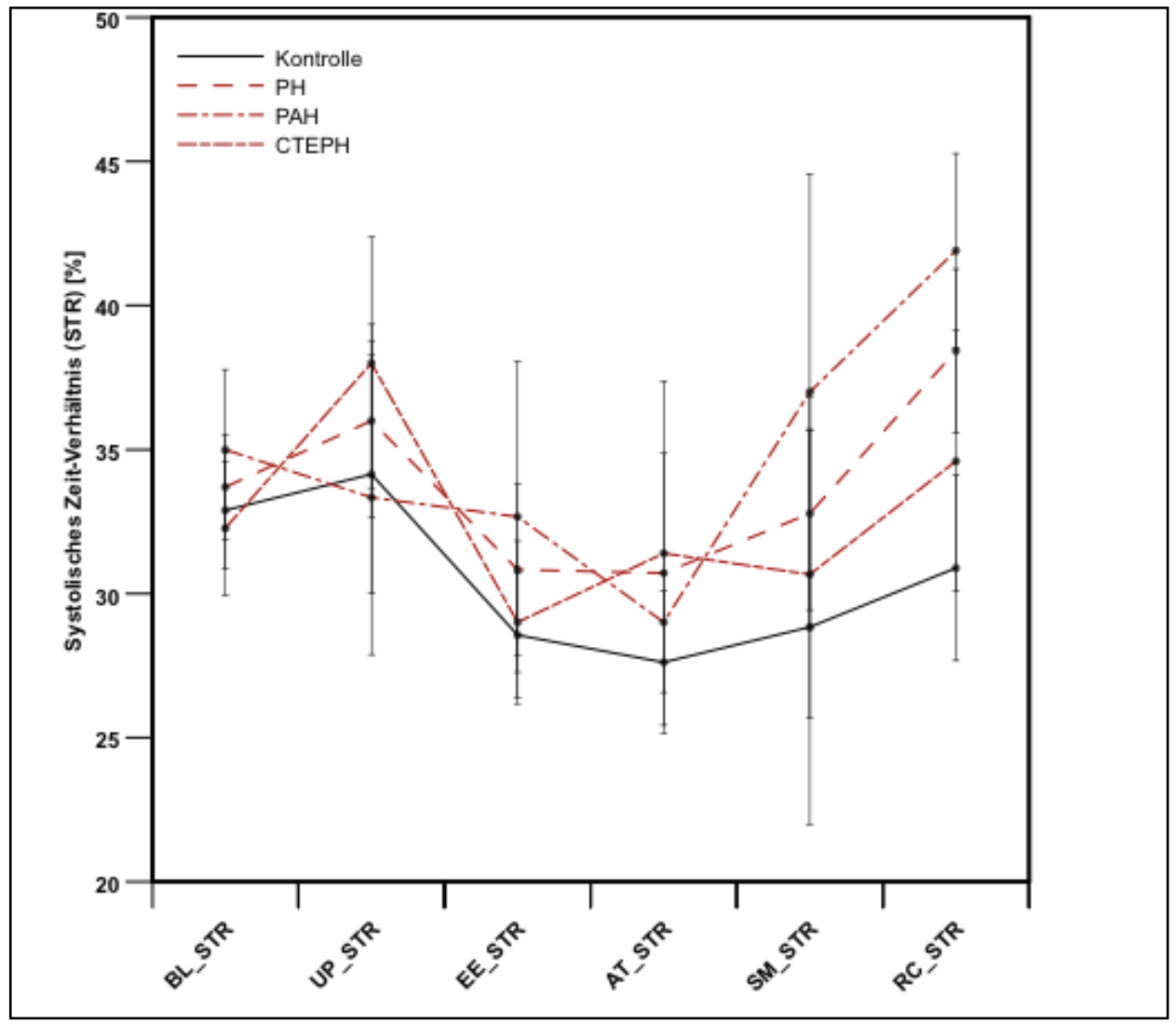

Abbildung 21: Darstellung des systolischen Zeitverhältnisses

\begin{tabular}{|l|c|c|c|c|}
\hline \multirow{2}{*}{ STR [\%] } & Kontrolle & Patienten & PAH & CTEPH \\
\cline { 2 - 5 } & MW \pm SD & MW \pm SD & MW \pm SD & MW \pm SD \\
\hline BL & $32,89 \pm 8,61$ & $33,70 \pm 8,67$ & $35,00 \pm 9,61$ & $32,27 \pm 7,71$ \\
\hline UP & $34,14 \pm 15,45$ & $36,00 \pm 12,52$ & $33,33 \pm 13,35$ & $38,00 \pm 12,38$ \\
\hline EE & $28,56 \pm 9,22$ & $30,83 \pm 10,25$ & $32,67 \pm 13,23$ & $29,00 \pm 6,93$ \\
\hline AT & $27,62 \pm 8,92$ & $30,71 \pm 10,98$ & $29,00 \pm 0,00$ & $31,40 \pm 13,37$ \\
\hline SM & $28,83 \pm 16,81$ & $32,78 \pm 12,10$ & $37,00 \pm 13,11$ & $30,67 \pm 12,23$ \\
\hline RC & $30,89 \pm 13,68$ & $38,43 \pm 12,99(*)$ & $41,91 \pm 11,18 \#$ & $34,60 \pm 14,32$ \\
\hline $\begin{array}{l}\text { (*) p }<0,1 \text { PH vs. Kontrolle } \\
\# \text { p }<0,05 \text { PAH vs. Kontrolle/ CTEPH vs. Kontrolle }\end{array}$ \\
\hline
\end{tabular}

Tabelle 32: Systolisches Zeitverhältnis zwischen Kontrolle und Patientengruppen

Das systolische Zeitverhältnis (STR), welches sich aus der Pre-Ejektionsperiode und der VET ableitet, beschreibt das Verhältnis der mechanischen zur elektrischen Systole. Die Pre-Ejektionsperiode spiegelt die Dauer der isovolumetrischen Kontraktion wieder. In Bezug auf das STR zeigen sich in beiden Gruppen zu allen 
Zeitpunkten der Untersuchung kaum signifikante Unterschiede. Lediglich im Bereich der Erholungsphase war das STR bei den PAH-Patienten im Vergleich zur Kontrolle signifikant erhöht $(30,89 \pm 13,68$ vs. $41,91 \pm 11,18 \%)$.

\subsection{Regressionsanalyse}

Zur Untersuchung der Zusammenhänge der mittels kardialer Bioimpedanz ermittelten Daten der Herzleistung wurden alle Messwerte, gruppiert nach KontrollProbanden und Patienten, mit dem Sauerstoffpuls zum jeweiligen Zeitpunkt in Verbindung gebracht. Es folgte eine lineare Regressionsanalyse und Vergleich der Regressionsgeraden.

\begin{tabular}{|c|l|c|c|c|}
\hline \multicolumn{2}{|c|}{} & Kontrolle & Patienten & \\
\cline { 3 - 5 } \multicolumn{2}{|c|}{ PFI } & MW \pm SD & MW \pm SD & p \\
\cline { 2 - 5 } & Ylope & $19,27 \pm 2,768$ & $6,946 \pm 4,423$ & 0.02709 \\
\hline \multirow{2}{*}{ ACI } & Slope & $6,647 \pm 1,027$ & $3,698 \pm 1,665$ & N/A \\
\cline { 2 - 5 } & Y-Achse & $89,27 \pm 14,84$ & $79,74 \pm 15,63$ & 0.1521 \\
\hline \multirow{2}{*}{ IC } & slope & $2,820 \pm 0,3325$ & $1,514 \pm 0,6564$ & $<0.0001$ \\
\cline { 2 - 5 } & Y-Achse & $26,13 \pm 4,847$ & $26,25 \pm 6,137$ & $<07954$ \\
\hline
\end{tabular}

Tabelle 33: Regressionsanalyse PFI, ACI, IC Vergleich zwischen Kontrollgruppe und Patienten 


\subsubsection{Akzelerationsindex}

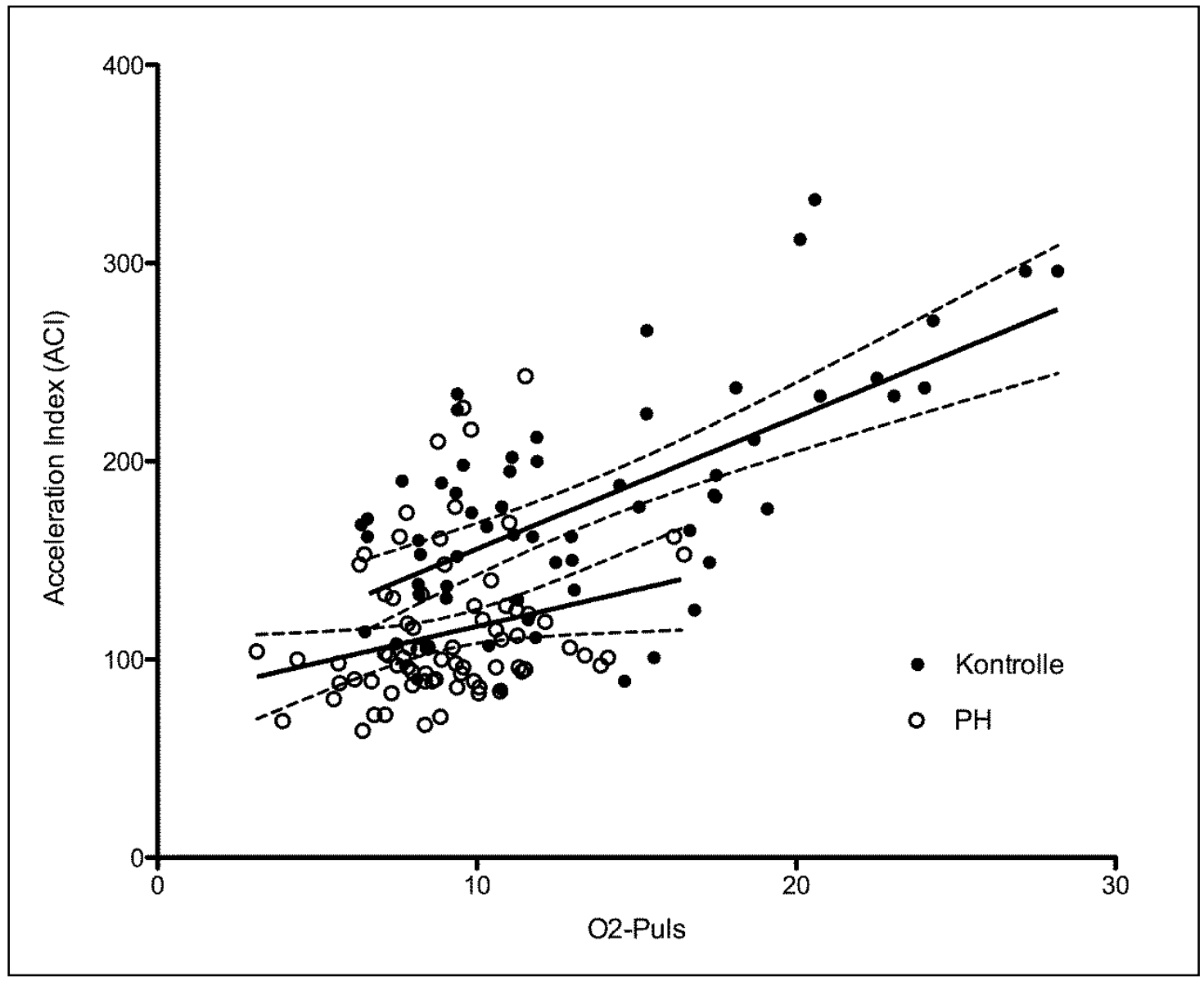

Abbildung 22: Korrelation zwischen dem Akzelerationsindex und dem Sauerstoffpuls

Der Akzelerationsindex (ACI) wies bei den gesunden Probanden ein signifikant höheres Ausgangsniveau (Y-Achsenabschnitt) als bei Patienten mit pulmonaler Hypertonie auf $(89,27 \pm 14,84$ vs. $79,74 \pm 15,63, p<0,0001)$. Dahingegen war die Entwicklung mit steigendem Sauerstoffpuls bei beiden Gruppen gleich $(6,647 \pm 1,027$ vs. $3,698 \pm 1,665, \mathrm{p}=0,1521)$. 


\subsubsection{Kontraktilitätsindex (IC)}

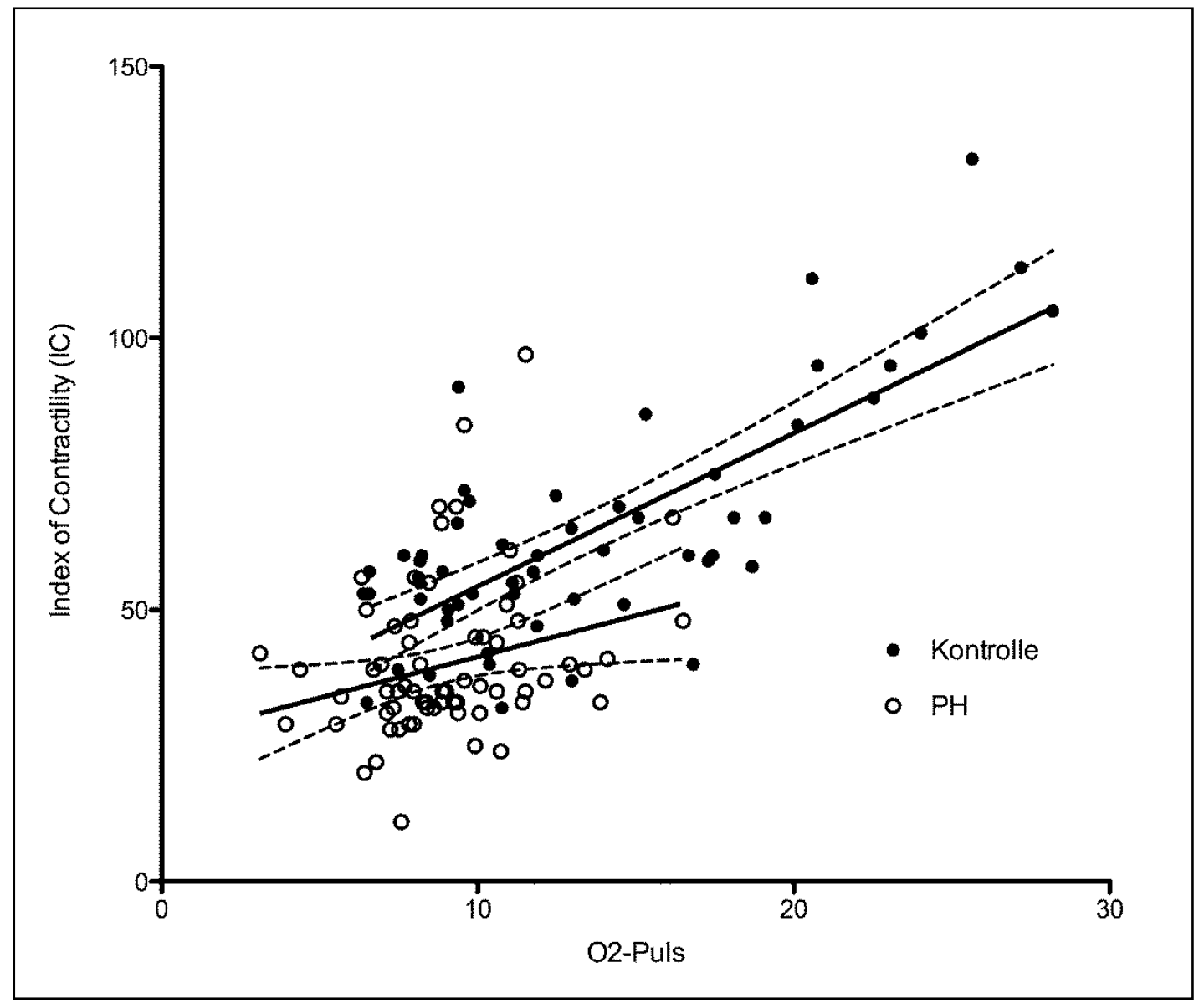

Abbildung 23: Korrelation zwischen dem Kontraktilitätsindex und dem Sauerstoffpuls

Beim Kontraktilitätsindex (IC) konnte ein geringer, jedoch signifikant niedrigerer Achsenabschnitt der Kontrollgruppe nachgewiesen werden (Kontrolle 26,13 $\pm 4,847$ vs. Patienten 26,25 $\pm 6,137, \mathrm{p}=0,001)$. Dies ging mit einem Trend $\mathrm{zu}$ unterschiedlichen Steigerungen des IC mit steigendem Sauerstoffpuls einher (Kontrolle 2,820 $\pm 0,3325$ vs. Patienten 1,514 $\pm 0,6564, p=0,0795$ ). 


\subsubsection{Peak Flow Index (PFI)}

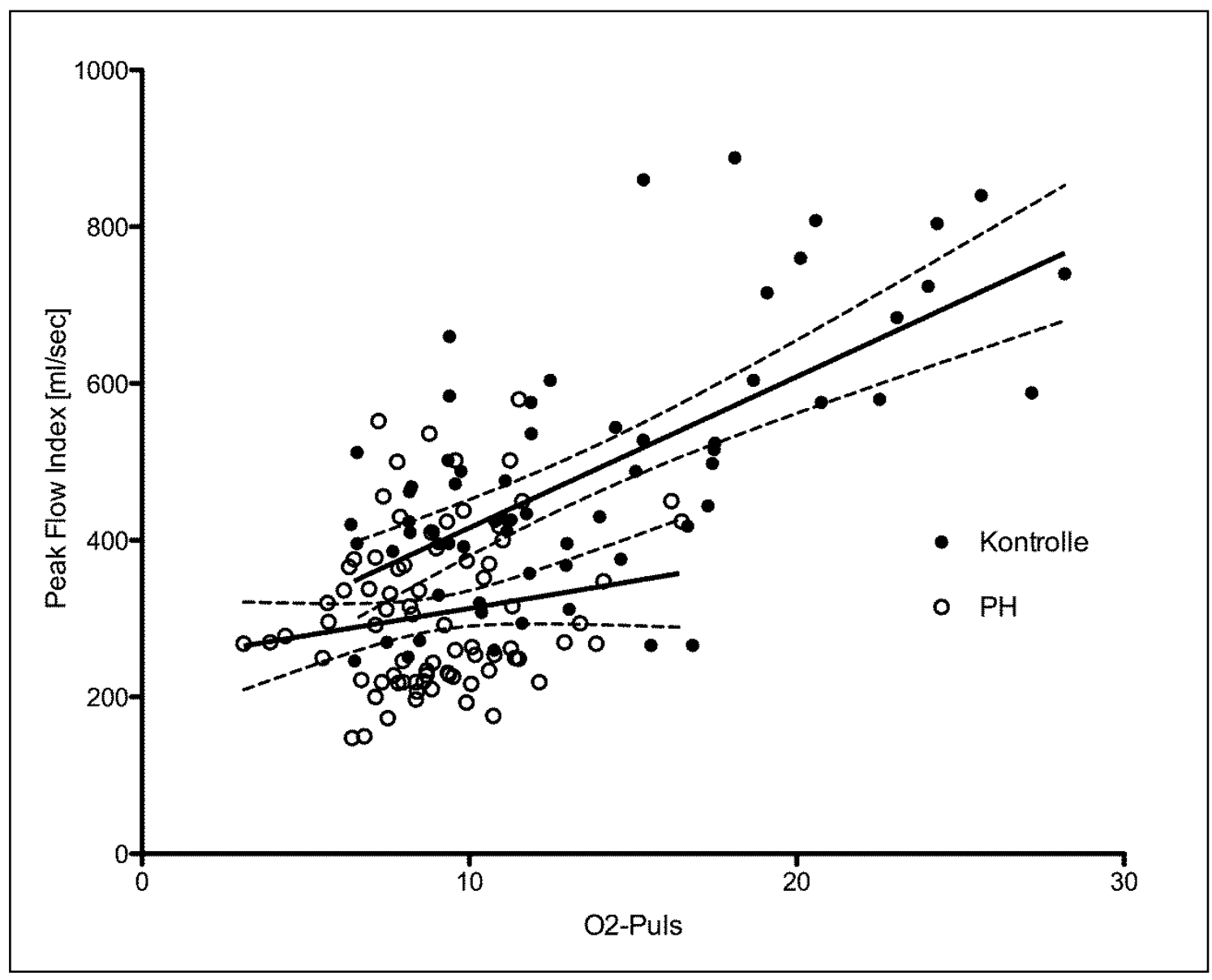

Abbildung 24: Korrelation zwischen dem Peak Flow Index und dem Sauerstoffpuls

Der Peak Flow Index zeigte zwischen den beiden Gruppen kein unterschiedliches

Niveau bezogen auf den Sauerstoffpuls (Y-Achsenabschnitte Kontrolle 223,2 $\pm 40,45$

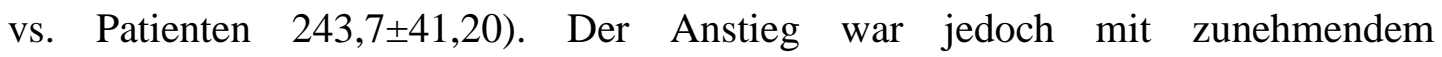
Sauerstoffpuls bei der Kontrollgruppe signifikant stärker als bei den Patienten

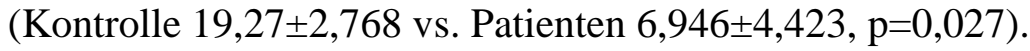




\section{Diskussion}

\subsection{Stellenwert der thorakalen Impedanzkardiographie}

Die Methode der thorakalen Bioimpedanz erhielt in der Literatur die letzten 30 Jahre viel Aufmerksamkeit. Diese Art der Bestimmung des Schlagvolumens und abgeleiteter Parameter wie beispielsweise des Herzzeitvolumens oder des Herzindexes vereint neben einem nicht-invasiven kontinuierlichen Monitoring auch Eigenschaften wie Untersucherunabhängigkeit und geringen Materialaufwand. Des Weiteren werden die Patienten keinem zusätzlichen Risiko durch invasive Diagnostik ausgesetzt. Die kardiale Bioimpedanzmessung ist ubiquitär einsetzbar, egal ob in der Praxis oder im Krankenhausalltag. Besonders ausgebildetes Personal ist zur Datenerhebung nicht erforderlich. Die geschilderten Vorteile begründen das Interesse für den Einsatz der Bioimpedanzmessung im Bereich der perioperativen Medizin.

Eine Erweiterung des Einsatzgebietes könnte beispielsweise auch in der Verlaufsbeurteilung der pulmonalen Hypertonie gesehen werden - hinsichtlich der Begutachtung eines Therapieerfolges oder zur Beurteilung neuer therapeutischer Ansätze. Nach wie vor ist die invasive Messung der pulmonal arteriellen Drücke, Herzzeitvolumens, pulmonal-kapillären Verschlussdruckes und pulmonal-vaskulären Widerstandes zwingend zur Diagnosestellung der pulmonalen Hypertonie erforderlich. In der Vergangenheit hatten sich vor allem der rechtsatriale Druck und das Herzzeitvolumen als diejenigen Parameter dargestellt, welche für die Prognose des Patienten von Bedeutung sind. Daher besteht ein erhebliches Interesse, diese Werte und möglicherweise weitere haemodynamische Kenndaten nicht-invasiv zu erheben, um den Krankheitsverlauf besser beurteilen zu können.

Das Vorgehen in Bezug auf die Berechnung des Herzzeitvolumens (HZV) oder des Schlagvolumens hat sich im Laufe der Jahre zunehmend verbessert, so dass die thorakale Bioimpedanzmessung innerhalb der letzten Jahre immer zuverlässiger geworden ist (Moshkovitz, Kaluski et al. 2004).

Einige Autoren fanden die Bestimmung des Herzzeitvolumens unter Belastung problematisch, sowohl aufgrund der thorakalen Atembewegungen als auch durch die Bewegungen, welche durch die Übung an sich ausgelöst wurden (Hetherington, Teo et al. 1985) (Veigl and Judy 1983) (Kobayashi, Andoh et al. 1978). Zur genaueren Signalableitung der Impedanzkardiographie veranlassten verschiedene Untersucher 
ihre Probanden zum kurzzeitigen Luftanhalten sowohl in Ruhe als auch unter Belastungsbedingungen (Edmunds, Godfrey et al. 1982) (Denniston, Maher et al. 1976) (Teo, Hetherington et al. 1985) (Hetherington, Teo et al. 1985) (Veigl and Judy 1983). Edmunds et al. dahingegen kamen $\mathrm{zu}$ dem Ergebnis, dass die Änderungen des Lungenvolumens beziehungsweise die Addition des expiratorischen Atemwegswiderstandes keinen Einfluss auf die Bestimmung des Herzzeitvolumens mittels Impedanzkardiographie unter Ruhe- sowie submaximalen Belastungsbedingungen haben (Edmunds, Godfrey et al. 1982). Einige Forscher nutzen sogar Belastungspausen zur Gewährleitung einer besseren Signalableitung (Teo, Hetherington et al. 1985) (Hetherington, Teo et al. 1985) (Kobayashi, Andoh et al. 1978) (Hatcher and Srb 1986). Dies erlaubte natürlich eine Reduktion der Messartefakte während der Belastungsuntersuchung. Jedoch zeigten weitere Studien, dass gerade in diesen Belastungspausen signifikante Änderungen von Schlagvolumen und der Herzfrequenz auftraten (Miles, Sawka et al. 1981). Hinsichtlich des Schlagvolumens zeigten sich in den Pausen keine relevanten Veränderungen während niedriger Leistungsstufen. Jedoch zeigte sich ein signifikanter Anstieg der Herzfrequenz, welche das Herzzeitvolumen deutlich beeinflusst. Während höherer Belastungsstufen ( $\mathrm{V} O 2>1 \mathrm{~L} / \mathrm{min}$ ) kam es zu einem Anstieg des Herzzeitvolumens (Miles and Gotshall 1989).

\subsection{Interpretation der hämodynamischen Veränderungen}

Eine Belastungssituation verlangt vom Körper kurzfristige Anpassungsreaktionen, um die Homöostase im Gleichgewicht halten zu können. Nach Wassermann kommt es primär zu einer peripheren Sauerstoffschuld infolge des Verbrauchs von ATP im Muskel und Mechanismen der ATP-Regeneration. Die Anpassungsreaktion des Körpers besteht aus der Erhöhung der Sauerstoffförderung durch das Herz-Kreislaufsystem und die Lunge.

Die Beurteilung dieser Anpassungsreaktion auf eine Belastung auf Basis von Werten, welche in Ruhe erhoben wurden, ist nur selten erfolgreich. Beispielsweise liegt bei einer Herzinsuffizienz ein Zustand vor, welcher es dem Patienten nicht erlaubt, das Herzzeitvolumen zu steigern, ohne die Füllungsdrücke des Ventrikels beizubehalten. Die Ejektionsfraktion wurde lange Zeit als Surrogat-Parameter des Schweregrades einer Herzinsuffizienz angesehen. Franciosa konnte in den 80er Jahren jedoch 
zeigen, dass keine Korrelation zwischen der Ejektionsfraktion und Parametern der körperlichen Leistungsfähigkeit besteht (Franciosa, Park et al. 1981).

Zur Objektivierung ist es daher erforderlich, den Patienten unter Belastung zu evaluieren. Die Spiroergometrie erlaubt sowohl eine Quantifizierung der Belastbarkeit, als auch Aussagen hinsichtlich der pathophysiologischen Einschränkungen, die der Belastungslimitierung zugrunde liegen.

In der vorliegenden Untersuchung konnte gezeigt werden, dass die Kombination aus Spiroergometrie mit kardialer Bioimpedanzmessung eine verbesserte Darstellung der hämodynamischen Parameter des linken Ventrikels unter Ruhebedingungen und bei unterschiedlichen Graden der körperlichen Belastung ermöglicht. Gegenüber der Beurteilung des Kreislaufsystems mit alleiniger Spiroergometrie hat die kardiale Bioimpedanz weniger Rahmenbedingungen zu beachten und ermöglicht somit eine direktere Messung der Kreislaufparameter.

Richard et al. führten eine nicht-invasive Bestimmung des Herzzeitvolumens mit dem Physio-Flow ${ }^{\circledR}$ einem Bioimpedanz-Monitoring-System unter Belastungsbedingungen durch. Sie konnten zeigen, dass die Bestimmung des Herzzeitvolumens während eines schrittweisen Belastungsanstieges klinisch zuverlässig und über mehrere Untersuchungen reproduzierbar war (Richard, Lonsdorfer-Wolf et al. 2001) (Hetherington, Haennel et al. 1986).

Gegenüber dem PhysioFlow ${ }^{\circledR}$ ist das BoMed-System nicht nur in der Lage, die Basisparameter des Kreislaufsystems zu erheben, sondern liefert zusätzlich Daten der Kontraktilität und des Blutflusses. Die Reproduzierbarkeit dieser Messungen konnte zumindest in Ruhe in den Vorversuchen dargelegt werden.

Erwartungsgemäß erreichten die Patienten mit einer pulmonalen Hypertonie in der hier durchgeführten Studie weitaus geringere Belastungsstufen als die gesunde Kontrollgruppe. Dies ließ sich auf eine reduzierte maximale Sauerstoffaufnahme zurückführen. Der ermittelte Herzindex war unter Ruhebedingungen und zu jedem Zeitpunkt der Belastung gegenüber der Kontrollgruppe erniedrigt. Nechwatal et al. führten vergleichende Untersuchungen des Herzzeitvolumens (HZV) mittels Thermodilution und Impedanzkardiographie unter Ergometerbelastung im Steady-State durch. Die Impedanzkardiographie konnte eine relative Änderung des HZV zuverlässig erfassen, Absolutwerte hingegen konnten nur näherungsweise ermittelt werden (Nechwatal, Bier et al. 1976). Zu einem ähnlichen Ergebnis kamen Boldt et al. bei dem Vergleich des HZVs mittels Thermodilutions- und 
Impedanzmethode bei operativ-intensivpflichtigen und herzchirurgischen Patienten. Die relativen Änderungen des Herzzeitvolumens waren, im Vergleich zu den Absolutgrößen, bei beiden Untersuchungsmethoden vergleichbar (Boldt, Kling et al. 1988).

Das Herzzeitvolumen setzt sich aus dem Schlagvolumen und der Herzfrequenz zusammen. In der vorliegenden Untersuchung konnte bei den Patienten ein geringerer Frequenzanstieg unter Belastung verzeichnet werden als in der Kontrollgruppe. Dies erscheint zunächst paradox, da eine linksventrikuläre Füllungsbehinderung einen inadäquat hohen Frequenzanstieg wie bei anderen Herzerkrankungen erwarten ließe (Wasserman 2005). Ein ähnliches Verhalten ist jedoch bereits bei Formen der dilatativen Kardiomyopathie beobachtet worden (Keteyian, Brawner et al. 1999). Es bleibt zu spekulieren, auf welchen Mechanismen diese chronotrope Inkompetenz beruht. Infrage kommen eine rechtsventrikuläre oder rechtsatriale Erhöhung der Wandspannung mit konsekutiver Beeinflussung des diastolischen Calcium-Stromes des Sinusknotens (Joung, Ogawa et al. 2009). Da die Probanden keinerlei bradykardisierende Medikation einnahmen (einschließlich Digitalis-Präparaten) konnte die Medikation als Ursache ausgeschlossen werden. Die spezifische Medikation (Endothelin-Rezeptor-Antagonisten, PhosphodiesteraseInhibitoren, Prostanoide) weist keine bradykardisierenden Eigenschaften auf (Sutsch, Kiowski et al. 1998) (Phillips, Kato et al. 2000) (Szczeklik, Szczeklik et al. 1980).

Die reduzierte Herzfrequenz wurde jedoch erst im Bereich der anaeroben Schwelle offensichtlich, während unter Ruhebedingungen keine signifikanten Unterschiede vorliegen, das Herzzeitvolumen jedoch bereits eingeschränkt war. Hierfür war ein reduziertes Schlagvolumen verantwortlich. Dieses war bereits in Ruhe reduziert und konnte unter Belastung nicht signifikant gesteigert werden. Gleichzeitig lag eine signifikant verminderte Ejektionsfraktion vor. Diese Größen stehen in direktem Zusammenhang mit dem diastolischen Füllungsvolumen, welches bei den Patienten ebenfalls deutlich vermindert war. Es kommt also $\mathrm{zu}$ einer Veränderung der linksventrikulären Volumina mit vermindertem Füllungsvolumen und daher reduzierter Vorspannung einerseits und vermindertem Schlagvolumen andererseits mit dem Ergebnis einer reduzierten Ejektionsfraktion. Aus echokardiografischen Untersuchungen ist bereits bekannt, dass es durch die rechtsventrikuläre Druckerhöhung zu einem Verschieben des interventrikulären Septums kommen kann, welches als „D-sign“, „,septal shift“ oder auch als „,septal bowing“ bezeichnet 
wird. Bisher existieren keine strukturierten Untersuchungen, in welcher Phase des Herzzyklus dieser septale Shift auftritt. Es wird in systolischen und diastolischen Shift unterschieden, das Maß der Deformierung jedoch lediglich als Exzentrizitätsindex des linken Ventrikels angegeben. Bei der pulmonalen Hypertonie können jedoch verschiedene Formen der interventrikulären Asynchronie auftreten. Einerseits durch einen Rechtsschenkelblock mit verzögerter Aktivierung des rechten Ventrikels, andererseits durch eine verzögerte und/ oder verkürzte rechtsventrikuläre Ejektionsphase durch die erhöhte Nachlast. Daher sind die genauen Auswirkungen dieser linksventrikulären Deformierung auf die Füllung oder die rechtsventrikuläre Ejektionsphase weitgehend ungeklärt. In dieser Studie wurden Reizleitungsstörungen vor Einschluss in die Studie ausgeschlossen, um dies als Fehlerquelle eliminieren zu können.

Während der Belastung steigt der pulmonalarterielle Druck ebenfalls an. Im Falle einer Rechtsherzinsuffizienz ist auch von einem Anstieg des rechtsventrikulären enddiastolischen Druckes und / oder des rechtsatrialen Druckes auszugehen. Diese Mechanismen können abhängig von den physikalischen Eigenschaften des Septums und den Veränderungen der Druckverhältnisse auch zur Entwicklung einer veränderten Füllung des linken Ventrikels führen, insbesondere, da die Diastolendauer unter Belastung abnimmt. Die Daten dieser Beobachtung wiesen auf eine Zunahme des enddiastolischen Volumen Index (EDI) bei leichter Belastung hin, welcher bei den Patienten etwas stärker ausfiel als bei den gesunden Probanden. Mit steigender Belastung war jedoch auch ein stärkerer Abfall zu verzeichnen, als es bei den Gesunden der Fall war. Die Aufklärung der Füllungsverhältnisse und des septalen Shifts bei pulmonaler Hypertonie bedarf weiterer Untersuchungen. Es wurden zwar Vorversuche zur Validierung der Messwerte mittels Echokardiografie durchgeführt, jedoch keine Echokardiografie unter Ergometerbelastung. Eine endgültige Klärung dieser Mechanismen ist daher in dieser Studie nicht möglich.

Eine verminderte Füllung des linken Ventrikels lassen die Beeinflussung des FranckStarling-Mechanismus und damit der Kontraktilität vermuten. Diese kann ferner im Falle eines septalen shifts auch durch eine interventrikuläre Asynchronie vermindert werden.

Das BoMed-System erlaubt die Erhebung verschiedener Indices der linksventrikulären Kontraktilität auf Basis der Flussentwicklung nach Öffnung der Aortenklappen. Zunächst wird der Peak Flow erhoben, welcher durch Kontraktilität 
und Nachlast bestimmt wird. Es war zu beobachten, dass Patienten mit pulmonaler Hypertonie einen weitaus geringeren Peak Flow in Ruhe hatten als die gesunden Probanden. Ferner stieg dieser unter Belastung nicht adäquat an. Dies ist insbesondere deshalb bemerkenswert, da die spezifische Medikation der Patienten auch eine peripher wirksame Komponente hat, welche durch Beeinflussung des NOSignalweges den vaskulären Gefäßwiderstand senkt. Es ist daher davon auszugehen, dass die Patienten nicht in der Lage waren, unter Belastung die myokardiale Kontraktilität in der gleichen Weise zu steigern wie bei gesunden Probanden. Ein Indikator ist die geringere Ejektionsfraktion.

Um die Ursachen genauer zu untersuchen, wurden Kontraktilitätsindex und Akzelerationsindex hinzugezogen. Der Akzelerationsindex (ACI) ist ein unabhängiger Parameter der Kontraktilität. Er ist direkt abhängig von der gemessenen Impedanz 20msec nach Öffnung der Aortenklappe und repräsentiert das bioelektrische Äquivalent des $\mathrm{dp} / \mathrm{dt}_{\max }$ der Hämodynamik. Er spiegelt daher vorlastunabhängig die myokardiale Kontraktilität und Inotropie wieder. Die Untersuchungen zeigten sowohl in Ruhe als auch unter Belastung einen reduzierten ACI bei Patienten mit pulmonaler Hypertonie.

Im Gegensatz hierzu ist der Kontraktilitätsindex (IC) von der Vorlast abhängig. Auch hier ließen sich sowohl in Ruhe als auch unter Belastung signifikant erniedrigte Werte der Patientengruppe feststellen. Aufgrund der gleichsinnigen Veränderung eines vorlastabhängigen und eines vorlastunabhängigen Parameters der Kontraktilität muss in Erwägung gezogen werden, dass die verminderte Kontraktilität myokardial bedingt ist. Die Möglichkeit einer reduzierten Füllung wurde bereits obenstehend diskutiert. Hinweise einer linksventrikulären Myokardveränderung ist bisher bei pulmonaler Hypertonie nicht beschrieben worden und eine pathophysiologische Herleitung ist nicht ersichtlich. Eine weitere Erklärungsmöglichkeit ist die zuvor beschriebene Asynchronie. Durch diese könnten sowohl eine verminderte Füllung als auch ein Kraftverlust in geometrischer Umformung verursacht werden und zu einer verminderten Kontraktilität führen (Stojnic, Brecker et al. 1992; Vonk-Noordegraaf, Marcus et al. 2005; Marcus, Gan et al. 2008).

Der Sauerstoffpuls wird in der Spiroergometrie gemeinhin als Äquivalent des Schlagvolumens betrachtet (Sun, Hansen et al. 2001). Er errechnet sich aus der Sauerstoffaufnahme pro Herzschlag. Dadurch wird die Herzfrequenz als Ursache für die Steigerung der Sauerstoffaufnahme während der Belastung eliminiert. Um den 
Zusammenhang der Kontraktilitätsveränderungen mit dem Schlagvolumen genauer $\mathrm{zu}$ untersuchen wurden alle Messwerte $\mathrm{zu}$ jeder Belastungsstufe nach Probandengruppe geordnet aufgetragen. Nach linearer Korrelation wurden die Regressionsgeraden der Gruppen statistisch verglichen. Hierbei konnte gezeigt werden, dass Patienten mit pulmonaler Hypertonie im Vergleich zu gesunden Probanden nicht in der Lage sind, die Kontraktilität im gleichen Maße zu steigern um das Schlagvolumen zu vergrößern. Die Messungen der Kontraktilitätsindizes sind bei dieser Methodik abhängig vom erreichten Effekt auf den Blutfluss aus dem Thoraxsegment. Sie sind daher nicht geeignet, Aussagen über die isovolumetrische Kontraktion oder die intracavitären Prozesse während der Kontraktion zu treffen. Die Ergebnisse können jedoch als Hinweis für die These einer ineffizienten Kontraktion interpretiert werden, da der messbare Effekt auf den Blutfluss reduziert ist. Die Möglichkeit eines Kraftverlustes durch geometrische Umformung infolge einer Asynchronie muss daher in Betracht gezogen werden und durch weitere Untersuchungen mittels Echokardiografie unter Belastung analysiert werden.

Gegen diese These steht jedoch das Argument, dass der Sauerstoffpuls nicht zwingend mit dem Schlagvolumen gleichgesetzt werden kann. Diese Annahme beruht auf der Voraussetzung, dass die Sauerstoffaufnahmekapazität konstant bleibt (Rolf F. Kroidl 2009). Durch die verstärkte Sauerstoffextraktion der Peripherie unter Belastung bei Patienten mit pulmonaler Hypertonie ist diese Grundannahme zu verwerfen. Untersuchungen zur Korrelation von Schlagvolumen und Sauerstoffpuls bei dieser Erkrankung sind bisher nicht publiziert und stellen ein weiteres Feld für zukünftige Studien dar.

\subsection{Limitierungen}

Messgenauigkeitseinschränkungen bei der Erhebung der hämodynamischen Parameter mittels des in dieser Studie genutzten Bioimpedanz-Monitoring-Systems könnten beispielsweise auftreten bei schlechter Elektrodenqualität, inkorrekter EKG-Triggerung, hoher Artefaktrate oder Interferenzen im Bereich von 50/60Hz.

Zur Eliminierung dieser Faktoren wurde auf eine optimale Platzierung der Elektroden mit Entfettung der Haut besonderer Wert gelegt. Als Elektroden dienten Silberchlorid-Elektroden gleichbleibender Qualität. Für die Messung wurden frische Sets verwendet um ein Austrocknen der Elektroden als Ursache eines schlechten Signal-Rauschverhältnisses auszuschließen. Um die EKG-Triggerung zu optimieren, 
verfügt das BoMed-System über eine gesonderte EKG-Ableitung, welche nicht zur Messwerterfassung der Bioimpedanz verwendet wird. Interferenzen mit dem Stromnetz konnten durch Erdung und 50Hz-Filter des Systems minimiert werden.

Die Messgenauigkeit kann durch erhöhte Blutdruckwerte (mittlere arterielle Druck (MAP) > $130 \mathrm{mmHg}$ ) dahingehend beeinflusst werden, dass das $\mathrm{HZV}$, das Schlagvolumen (SV), der Kontraktilitätsindex (IC), der Peak Flow (PF) und der Akzelerationsindex (ACI) unterschätzt werden. Eine Aortenklappeninsuffizienz wiederum führt zu einer Überschätzung des HZV, des SV, des CI, des PF sowie des ACI. Bei dem Vorliegen einer akuten Mitralklappeninsuffizienz kann die Ejektionsfraktion (EF) über- und somit das enddiastolische Volumen (EDV) zu niedrig dargestellt werden. Umgekehrt verhält es sich bei dem Bestehen von Schenkelblöcken. Alle diese Vitien sowie Schenkelblöcke lagen in dem von uns untersuchten Patientenkollektiv nicht vor. Die vorherige Echokardiografie und Elektrokardiografie schloss andere kardiale Erkrankungen aus.

Limitierungen bei der Erhebung der hämodynamischen Parameter mittels des Bioimpedanz-Monitoring-Systems sind beispielsweise in der Berechnung der Auswurfleistung sowie bei den Ventrikelvolumina zu suchen. Deren Ermittlung erfolgt über Zeitintervallformeln und Änderungen in der Impedanz. Dies bedeutet, es werden normale Füllungsraten vermutet, welche jedoch bei Patienten mit einer pulmonalen Hypertonie nicht anzunehmen sind. Die Ermittlung der Fluss- sowie Kontraktilitätsparameter basiert hingegen ausschließlich auf Impedanzmessungen und sind somit durch diese Änderungen unverfälscht.

\subsection{Zusammenfassung}

Zusammenfassend konnte in dieser Studie mit Hilfe der kardialen Bioimpedanzmessung gezeigt werden, dass die Patienten mit einer pulmonalen Hypertonie nicht in der Lage waren, ihre Kontraktilität sowie ihre Herzfrequenz im Vergleich zur Kontrollgruppe unter Belastung adäquat $\mathrm{zu}$ steigern. Die Inotropie-Parameter, wie der Peak Flow und der Akzelerationsindex, waren im Patientenkollektiv im Vergleich zu den gesunden Probanden erniedrigt. Es zeigte sich sowohl in Ruhe als auch unter Belastungsbedingungen eine Reduktion der linksventrikulären Performance. Die reduzierten Inotropie-Indizes lassen auf eine globale kardiale Beeinträchtigung des Herzens bei der pulmonalen Hypertonie schließen, welche über eine Rechtsherzbelastung hinauszugehen scheint. Dies macht 
sich dadurch bemerkbar, dass bei Steigerung der Inotropie der Sauerstoffpuls als Äquivalent des Schlagvolumens nicht in gleichem Maße ansteigt, wie das beim Gesunden der Fall ist. Mögliche Ursachen sind ein septaler Shift und eine Asynchronie durch veränderte Druckverhältnisse und sind Ziel weiterer Untersuchungen.

Zusammenfassend gesehen stellt die kardiale Bioimpedanz eine Möglichkeit der nicht-invasiven Beurteilung der haemodynamischen Veränderungen bei pulmonaler Hypertonie sowohl in Ruhe als auch unter Belastung dar. Die zusätzlichen Parameter der linksventrikulären Kontraktilität ermöglichen Einblicke in die ventrikuläre Interaktion, welche zunehmend in das Blickfeld der Forschung bei diesem Krankheitsbild kommt. 


\section{Abbildungsverzeichnis}

Abbildung 1: Imbalance zwischen vasodilatativen und vasokonstriktiven Mediatoren .......... 8

Abbildung 2: Elektrodenanordnung zum Monitoring der kardialen Bioimpedanz............... 25

Abbildung 3: Verteilung und Anzahl der Studienteilnehmer ..................................... 42

Abbildung 4: Durchschnittsalter der Studienteilnehmer............................................ 42

Abbildung 5: Vergleich der maximalen Sauerstoffaufnahme zwischen Patienten- und

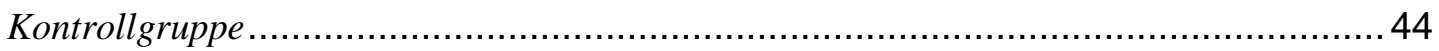

Abbildung 6: Vergleich der maximalen Leistung zwischen Patienten- und Kontrollgruppe 44

Abbildung 7: Darstellung der Sauerstoffaufnahme zum jeweiligen Belastungszeitpunkt ..... 46

Abbildung 8: Darstellung der Sauerstoffaufnahme pro $\mathrm{kg} K G$................................... 47

Abbildung 9: Darstellung $\dot{V E} / \dot{V} C O 2$ an den jeweiligen Belastungszeitpunkten .................48

Abbildung 10: Vergleich des Herzindexes zwischen Patienten- und Kontrollgruppe zu den unterschiedlichen Belastungszeitpunkten ......................................................... 49

Abbildung 11: Vergleich der Herzfrequenz zwischen Patienten- und Kontrollgruppe zu den unterschiedlichen Belastungszeitpunkten ....................................................50

Abbildung 12: Vergleich des Schlagvolumenindexes zwischen Patienten- und Kontrollgruppe zu den jeweiligen Belastungszeitpunkten .........................................51 Abbildung 13: Vergleich der Ejektionsfraktion zwischen Patienten- und Kontrollgruppe zu den jeweiligen Belastungszeitpunkten ............................................................ 52

Abbildung 14: Darstellung des enddiastolischen Volumenindexes zwischen Patienten- und Kontrollgruppe zu den jeweiligen Belastungszeitpunkten ........................................53

Abbildung 15: Darstellung des Akzelerationsindexes zwischen Patienten- und Kontrollgruppe zu den jeweiligen Belastungszeitpunkten ........................................ 54

Abbildung 16: Darstellung des Peak Flow Indexes zwischen Patienten- und Kontrollgruppe zu den jeweiligen Belastungszeitpunkten ........................................55

Abbildung 17: Darstellung des Ejektionsverhältnisses zwischen Patienten- und Kontrollgruppe zu den jeweiligen Belastungszeitpunkten ..........................................56

Abbildung 18: Darstellung des Kontraktilitätsindexes .......................................... 57

Abbildung 19: Darstellung des thorakalen Flüssigkeitsindexes...................................58

Abbildung 20: Darstellung der ventrikulären Ejektionszeit .......................................59

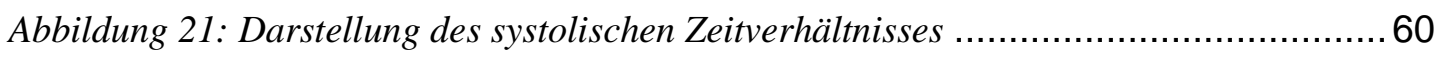

Abbildung 22: Korrelation zwischen dem Akzelerationsindex und dem Sauerstoffpuls....... 62

Abbildung 23: Korrelation zwischen dem Kontraktilitätsindex und dem Sauerstoffpuls .....63

Abbildung 24: Korrelation zwischen dem Peak Flow Index und dem Sauerstoffpuls...........64 


\section{Tabellenverzeichnis}

Tabelle 1: Symptome der pulmonalen Hypertonie (Rich, Dantzker et al. 1987)................... 4

Tabelle 2: klinische Klassifikation der pulmonalen Hypertonie (Galie, Hoeper et al. 2009) 7

Tabelle 3: WHO- Klassifikation des funktionellen Schweregrades bei Patienten mit

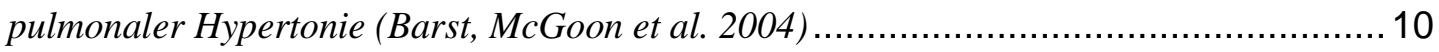

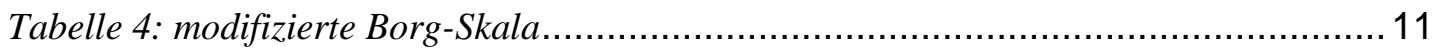

Tabelle 5: EKG- Befunde bei Rechtsherzbelastung.................................................. 12

Tabelle 6: Hämodynamik der PH (Opitz, Blindt et al.)................................................... 19

Tabelle 7: Übersicht der anthropomorphen Parameter................................................ 26

Tabelle 8: Hämodynamische Eigenschaften des Patientenkollektivs ............................... 27

Tabelle 9: Parameter der kardialen Bioimpedanz ......................................................... 28

Tabelle 10: Normwerte Herzfrequenz und ventrikuläre Ejektionszeit (1992) .................... 33

Tabelle 11: Normwerte Herzfrequenz, VET, Herzfrequenzperiode, ER (1992) .................. 34

Tabelle 12: Parameter der Spiroergometrie ............................................................. 36

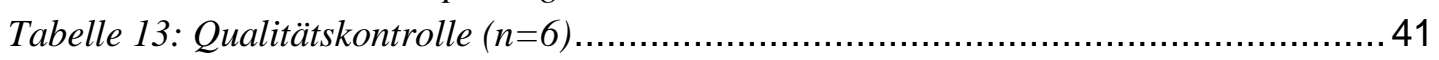

Tabelle 14: Übersicht der Basischarakteristika ....................................................... 41

Tabelle 15: Medikation zum Untersuchungszeitpunkt ................................................ 43

Tabelle 16: Anzahl der Medikation zum Untersuchungszeitpunkt ................................. 43

Tabelle 17: maximale Sauerstoffaufnahme und Leistung der Patienten im Vergleich zur

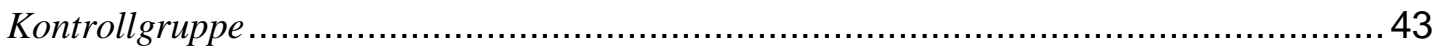

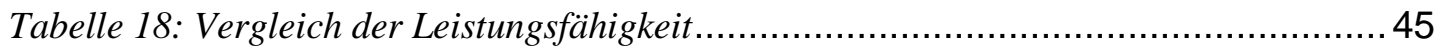

Tabelle 19: Sauerstoffaufnahme Kontrolle und Patientengruppen...................................46

Tabelle 20: $\dot{V} E / \dot{V} \mathrm{CO}_{2}$ Kontrolle und Patientengruppen ........................................... 48

Tabelle 21: Herzindex der Patienten- und Kontrollgruppe an den jeweiligen

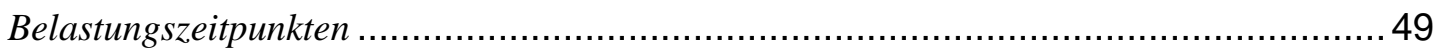

Tabelle 22: Herzfrequenz der Patienten- und Kontrollgruppe an den jeweiligen

Belastungszeitpunkten .................................................................................. 50

Tabelle 23: Schlagvolumenindex der Patienten- und Kontrollgruppean den jeweiligen

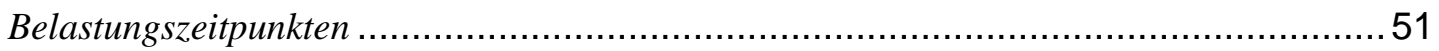

Tabelle 24: Ejektionsfraktion der Patienten- und Kontrollgruppe an den jeweiligen

Belastungszeitpunkten ............................................................................... 52

Tabelle 25: Enddiastolischer Volumenindex der Patienten- und Kontrollgruppe an den

jeweiligen Belastungszeitpunkten ..................................................................... 53

Tabelle 26: Akzelerationsindex der Patienten- und Kontrollgruppe an den jeweiligen

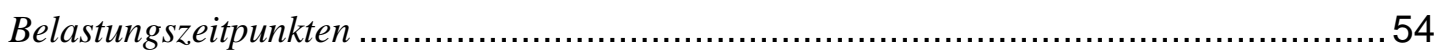

Tabelle 27: Peak Flow Index der Patienten- und Kontrollgruppe an den jeweiligen

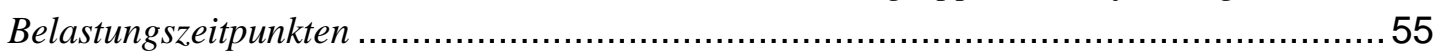

Tabelle 28: Ejektionsverhältnis der Patienten- und Kontrollgruppe an den jeweiligen

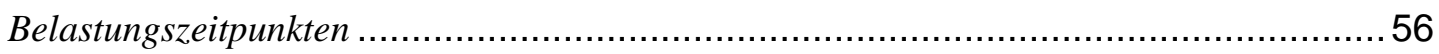

Tabelle 29: Kontraktilitätsindex zwischen Kontrolle und Patientengruppen ..................... 57

Tabelle 30: Thorakaler Flüssigkeitsindex zwischen Kontrolle und Patientengruppen .........58

Tabelle 31: Ventrikuläre Ejektionszeit zwischen Kontrolle und Patientengruppen...............59

Tabelle 32: Systolisches Zeitverhältnis zwischen Kontrolle und Patientengruppen .............60

Tabelle 33: Regressionsanalyse PFI, ACI, IC Vergleich zwischen Kontrollgruppe und

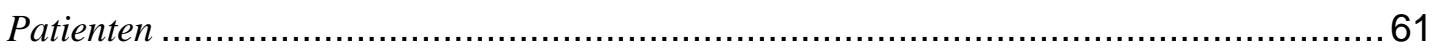

Tabelle 34: Blutgasanalyse Kontrolle und Patientengruppen .................................. XVII

Tabelle 35: Lungenfunktion Kontrolle und Patientengruppen .................................... XVII 


\section{Formelverzeichnis}

Formel 1: Berechnung des Peak Flow 28

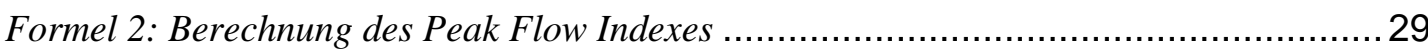

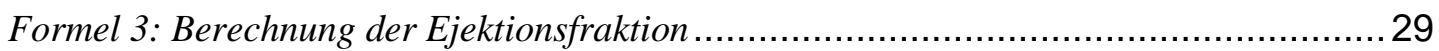

Formel 4: Algorithmus der linksventrikulären Ejektionsfraktion von L. Capan (Capan 1987)

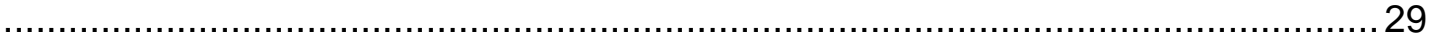

Formel 5: Berechnung der Ejektionsfraktion nach L.Capan (Capan 1987) .....................29

Formel 6: Berechnung des enddiastolischen Volumens.................................. 30

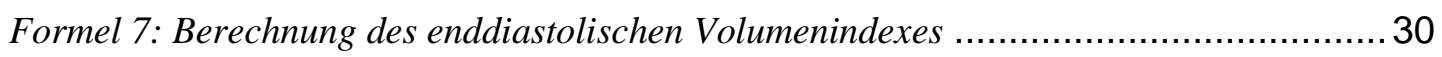

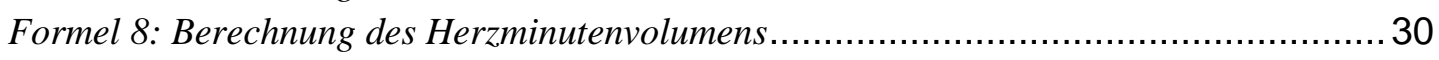

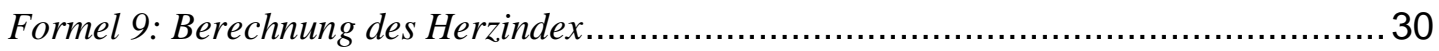

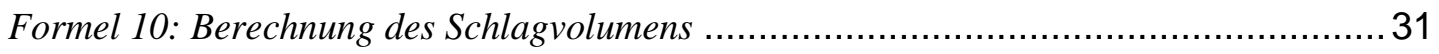

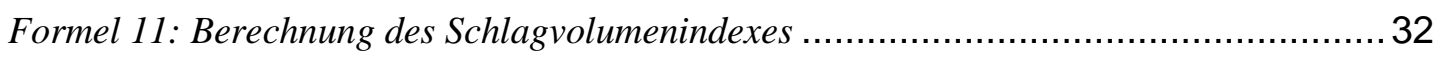

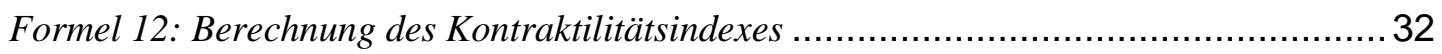

Formel 13: Berechnung der ventrikulären Ejektionszeit.................................... 33

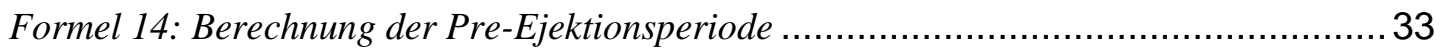

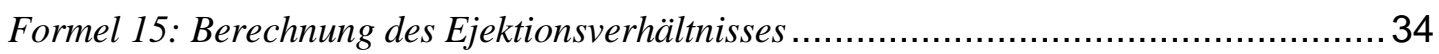

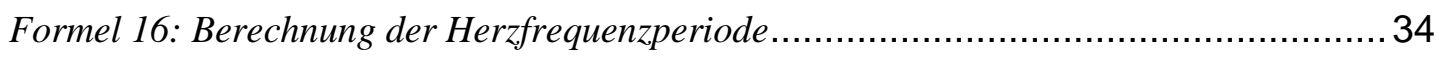

Formel 17: Berechnung des systolisches Zeitverhältnisses ............................... 34 


\section{Literaturverzeichnis}

(1992). BoMed CDDP System NCCOM3-R7S Cardiodynamic Monitor Service Manual

(2002). "ATS statement: guidelines for the six-minute walk test." Am J Respir Crit Care Med 166(1): 111-7.

Atzler, L. (1932). "Über ein neues Verfahren zur Darstellung der Herztätigkeit

(Dielektrographie)." European Journal of Applied Physiology and Occupational Physiology 5: 636-680.

Badesch, D. B., H. C. Champion, et al. (2009). "Diagnosis and assessment of pulmonary arterial hypertension." J Am Coll Cardiol 54(1 Suppl): S55-66.

Barst, R. J., J. S. Gibbs, et al. (2009). "Updated evidence-based treatment algorithm in pulmonary arterial hypertension." J Am Coll Cardiol 54(1 Suppl): S78-84.

Barst, R. J., M. McGoon, et al. (2004). "Diagnosis and differential assessment of pulmonary arterial hypertension." J Am Coll Cardiol 43(12 Suppl S): 40S47S.

Barton, M. and M. Yanagisawa (2008). "Endothelin: 20 years from discovery to therapy." Can J Physiol Pharmacol 86(8): 485-98.

Beaver, W. L., K. Wasserman, et al. (1986). "A new method for detecting anaerobic threshold by gas exchange." J Appl Physiol 60(6): 2020-7.

Behr, J., M. M. Borst, et al. (2005). "[A role for combination therapy in pulmonary arterial hypertension]." Pneumologie 59(10): 730-5.

Bernstein, D. P. (1986). "A new stroke volume equation for thoracic electrical bioimpedance: theory and rationale." Crit Care Med 14(10): 904-9.

Boldt, J., D. Kling, et al. (1988). "[Non-invasive versus invasive cardiovascular monitoring. Determination of stroke volume and pulmonary hydration using a new bioimpedance monitor]." Anaesthesist 37(4): 218-23.

Bonderman, D., P. Wexberg, et al. (2011). "A noninvasive algorithm to exclude precapillary pulmonary hypertension." Eur Respir J 37(5): 1096-103.

Borg, G. A. (1982). "Psychophysical bases of perceived exertion." Med Sci Sports Exerc 14(5): 377-81.

Capan, L. M. B., D.P.; Sanger, J. and Turndorf, H. (1987). "Measurement of Ejection Fraction By Bioimpedance Method." Critical Care Medicine 15(4): 402.

Castor, M. J., Altmayer P., Molter G. (1989). "A Computer Aided Non-invasiv Monitoring System with graphic Display of Hemodynamic Parameters Recorded On-line." Biomedizinische Technik 34: 262-267.

Christman, B. W., C. D. McPherson, et al. (1992). "An imbalance between the excretion of thromboxane and prostacyclin metabolites in pulmonary hypertension." N Engl J Med 327(2): 70-5.

Denniston, J. C., J. T. Maher, et al. (1976). "Measurement of cardiac output by electrical impedance at rest and during exercise." J Appl Physiol 40(1): 91-5.

Dumitrescu, D., R. J. Oudiz, et al. (2010). "Developing pulmonary vasculopathy in systemic sclerosis, detected with non-invasive cardiopulmonary exercise testing." PLoS One 5(12): e14293.

Dumitrescu, D. and S. Rosenkranz (2008). "[The role of cardiopulmonary exercise testing in the assessment of pulmonary hypertension]." Dtsch Med Wochenschr 133 Suppl 6: S176-9. 
Edmunds, A. T., S. Godfrey, et al. (1982). "Cardiac output measured by transthoracic impedance cardiography at rest, during exercise and at various lung volumes." Clin Sci (Lond) 63(2): 107-13.

Eggers, K. M., M. Nygren, et al. "High-sensitive troponin T and I are related to invasive hemodynamic data and mortality in patients with left-ventricular dysfunction and precapillary pulmonary hypertension." Clin Chim Acta 412(17-18): 1582-8.

Ellestad, M. H. and M. K. Wan (1975). "Predictive implications of stress testing. Follow-up of 2700 subjects after maximum treadmill stress testing." Circulation 51(2): 363-9.

Erdmann, E. (2011). "Klinische Kardiologie: Krankheiten des Herzens, des Kreislaufs und der herznahen Gefäße." 8. Auflage Kapitel 11.

Fedullo, P. F., W. R. Auger, et al. (2001). "Chronic thromboembolic pulmonary hypertension." N Engl J Med 345(20): 1465-72.

Fijalkowska, A., M. Kurzyna, et al. (2006). "Serum N-terminal brain natriuretic peptide as a prognostic parameter in patients with pulmonary hypertension." Chest 129(5): 1313-21.

Forfia, P. R., M. R. Fisher, et al. (2006). "Tricuspid annular displacement predicts survival in pulmonary hypertension." Am J Respir Crit Care Med 174(9): 1034-41.

Franciosa, J. A., M. Park, et al. (1981). "Lack of correlation between exercise capacity and indexes of resting left ventricular performance in heart failure." Am J Cardiol 47(1): 33-9.

Fuster, V., P. M. Steele, et al. (1984). "Primary pulmonary hypertension: natural history and the importance of thrombosis." Circulation 70(4): 580-7.

Gaine, S. P. and L. J. Rubin (1998). "Primary pulmonary hypertension." Lancet 352(9129): 719-25.

Galie, N., B. H. Brundage, et al. (2009). "Tadalafil therapy for pulmonary arterial hypertension." Circulation 119(22): 2894-903.

Galie, N., H. A. Ghofrani, et al. (2005). "Sildenafil citrate therapy for pulmonary arterial hypertension." N Engl J Med 353(20): 2148-57.

Galie, N., M. M. Hoeper, et al. (2009). "Guidelines for the diagnosis and treatment of pulmonary hypertension." Eur Respir J 34(6): 1219-63.

Galie, N., L. Rubin, et al. (2008). "Treatment of patients with mildly symptomatic pulmonary arterial hypertension with bosentan (EARLY study): a doubleblind, randomised controlled trial." Lancet 371(9630): 2093-100.

Ghofrani, H. A., O. Distler, et al. "[Treatment of pulmonary arterial hypertension (PAH): recommendations of the Cologne Consensus Conference 2010]." Dtsch Med Wochenschr 135 Suppl 3: S87-101.

Giaid, A. and D. Saleh (1995). "Reduced expression of endothelial nitric oxide synthase in the lungs of patients with pulmonary hypertension." $\underline{\mathrm{N} \text { Engl J }}$ Med 333(4): 214-21.

Goeckenjan, G., H. Sitter, et al. (2010). "Prevention, diagnosis, therapy, and followup of lung cancer: interdisciplinary guideline of the German Respiratory Society and the German Cancer Society." Pneumologie 65(1): 39-59.

Grünig, E., A. Barner, et al. (2010). "[Non-invasive diagnosis of pulmonary hypertension: ESC/ERS Guidelines with commentary of the Cologne Consensus Conference 2010]." Dtsch Med Wochenschr 135 Suppl 3: S67-77. 
Grünig, E. and S. Ley (2008). "[Echocardiography and magnetic resonance imaging in patients suffering from pulmonary arterial hypertension]." Dtsch Med Wochenschr 133 Suppl 6: S173-5.

Hansen, J. E., G. Ulubay, et al. (2007). "Mixed-expired and end-tidal CO2 distinguish between ventilation and perfusion defects during exercise testing in patients with lung and heart diseases." Chest 132(3): 977-83.

Hassoun, P. M., L. Mouthon, et al. (2009). "Inflammation, growth factors, and pulmonary vascular remodeling." J Am Coll Cardiol 54(1 Suppl): S10-9.

Hatcher, D. D. and O. D. Srb (1986). "Comparison of two noninvasive techniques for estimating cardiac output during exercise." J Appl Physiol 61(1): 155-9.

Heresi, G. A., W. H. Tang, et al. "Sensitive cardiac troponin I predicts poor outcomes in pulmonary arterial hypertension." Eur Respir J 39(4): 939-44.

Hetherington, M., R. Haennel, et al. (1986). "Importance of considering ventricular function when prescribing exercise after acute myocardial infarction." Am J Cardiol 58(10): 891-5.

Hetherington, M., K. K. Teo, et al. (1985). "Use of impedance cardiography in evaluating the exercise response of patients with left ventricular dysfunction." Eur Heart J 6(12): 1016-24.

Hill, N. S., Farber H. W. (2008). Pulmonary Hypertension, Humana Press.

Hoeft, M., Pasch (2008). Monitoring in Anästhesie und Intensivmedizin. Berlin, Springer.

Hoeper, M. M., I. Markevych, et al. (2005). "Goal-oriented treatment and combination therapy for pulmonary arterial hypertension." Eur Respir J 26(5): 858-63.

Hoeper, M. M., M. W. Pletz, et al. (2007). "Prognostic value of blood gas analyses in patients with idiopathic pulmonary arterial hypertension." Eur Respir J 29(5): 944-50.

Humbert, M., O. Sitbon, et al. (2006). "Pulmonary arterial hypertension in France: results from a national registry." Am J Respir Crit Care Med 173(9): 1023-30.

Humbert, M., O. Sitbon, et al. (2004). "Treatment of pulmonary arterial hypertension." N Engl J Med 351(14): 1425-36.

Itoh, H., K. Taniguchi, et al. (1990). "Evaluation of severity of heart failure using ventilatory gas analysis." Circulation 81(1 Suppl): II31-7.

Jennings, G. L. and M. D. Esler (1990). "Circulatory regulation at rest and exercise and the functional assessment of patients with congestive heart failure." Circulation 81(1 Suppl): II5-13.

Jing, Z. C., X. Jiang, et al. (2009). "Iloprost for pulmonary vasodilator testing in idiopathic pulmonary arterial hypertension." Eur Respir J 33(6): 1354-60.

Joung, B., M. Ogawa, et al. (2009). "The calcium and voltage clocks in sinoatrial node automaticity." Korean Circ J 39(6): 217-22.

Keim, H. J., J. M. Wallace, et al. (1976). "Impedance cardiography for determination of stroke index." J Appl Physiol 41(5 Pt. 1): 797-9.

Keteyian, S. J., C. A. Brawner, et al. (1999). "Effects of exercise training on chronotropic incompetence in patients with heart failure." Am Heart J 138(2 Pt 1): 233-40.

Kobayashi, Y., Y. Andoh, et al. (1978). "Impedance cardiography for estimating cardiac output during submaximal and maximal work." J Appl Physiol 45(3): 459-62.

Kubicek, W. G., J. N. Karnegis, et al. (1966). "Development and evaluation of an impedance cardiac output system." Aerosp Med 37(12): 1208-12. 
Lele, S. S., G. Scalia, et al. (1994). "Mechanism of exercise hypotension in patients with ischemic heart disease. Role of neurocardiogenically mediated vasodilation." Circulation 90(6): 2701-9.

Marcus, J. T., C. T. Gan, et al. (2008). "Interventricular mechanical asynchrony in pulmonary arterial hypertension: left-to-right delay in peak shortening is related to right ventricular overload and left ventricular underfilling." $\mathrm{J} \mathrm{Am}$ Coll Cardiol 51(7): 750-7.

Masuyama, T., K. Kodama, et al. (1986). "Continuous-wave Doppler echocardiographic detection of pulmonary regurgitation and its application to noninvasive estimation of pulmonary artery pressure." Circulation 74(3): 484-92.

Mehta, S. (2003). "Sildenafil for pulmonary arterial hypertension: exciting, but protection required." Chest 123(4): 989-92.

Miles, D. S. and R. W. Gotshall (1989). "Impedance cardiography: noninvasive assessment of human central hemodynamics at rest and during exercise." Exerc Sport Sci Rev 17: 231-63.

Miles, D. S., M. N. Sawka, et al. (1981). "Estimation of cardiac output by electrical impedance during arm exercise in women." J Appl Physiol 51(6): 1488-92.

Miyamoto, S., N. Nagaya, et al. (2000). "Clinical correlates and prognostic significance of six-minute walk test in patients with primary pulmonary hypertension. Comparison with cardiopulmonary exercise testing." Am J Respir Crit Care Med 161(2 Pt 1): 487-92.

Morrell, N. W., S. Adnot, et al. (2009). "Cellular and molecular basis of pulmonary arterial hypertension." J Am Coll Cardiol 54(1 Suppl): S20-31.

Moshkovitz, Y., E. Kaluski, et al. (2004). "Recent developments in cardiac output determination by bioimpedance: comparison with invasive cardiac output and potential cardiovascular applications." Curr Opin Cardiol 19(3): 229-37.

Myers, J., N. Buchanan, et al. (1991). "Comparison of the ramp versus standard exercise protocols." J Am Coll Cardiol 17(6): 1334-42.

Myers, J. and V. F. Froelicher (1993). "Exercise testing. Procedures and implementation." Cardiol Clin 11(2): 199-213.

Naggar, C. Z., D. B. Dobnik, et al. (1975). "Accuracy of the stroke index as determined by the transthoracic electrical impedance method." Anesthesiology 42(2): 201-5.

Nechwatal, W., P. Bier, et al. (1976). "[The noninvasive determination of cardiac output by means of impedance cardiography. Comparative evaluation with a thermal dilution technique (author's transl)]." Basic Res Cardiol 71(5): 54252.

Olschewski, H., M. M. Hoeper, et al. (2006). "[Diagnosis and therapy of chronic pulmonary hypertension]." Pneumologie 60(12): 749-71.

Olschewski, H., G. Simonneau, et al. (2002). "Inhaled iloprost for severe pulmonary hypertension." N Engl J Med 347(5): 322-9.

Opitz, C. F., R. Blindt, et al. "[Pulmonary hypertension: hemodynamic evaluation: hemodynamic evaluation - recommendations of the Cologne Consensus Conference 2010]." Dtsch Med Wochenschr 135 Suppl 3: S78-86.

Paciocco, G., F. J. Martinez, et al. (2001). "Oxygen desaturation on the six-minute walk test and mortality in untreated primary pulmonary hypertension." Eur Respir J 17(4): 647-52.

Phillips, B. G., M. Kato, et al. (2000). "Sympathetic activation by sildenafil." Circulation 102(25): 3068-73. 
Poehlman, E. T., T. L. McAuliffe, et al. (1990). "Influence of age and endurance training on metabolic rate and hormones in healthy men." Am J Physiol 259(1 Pt 1): E66-72.

Putman, C. T., L. L. Spriet, et al. (1993). "Pyruvate dehydrogenase activity and acetyl group accumulation during exercise after different diets." Am J Physiol 265(5 Pt 1): E752-60.

Raymond, R. J., A. L. Hinderliter, et al. (2002). "Echocardiographic predictors of adverse outcomes in primary pulmonary hypertension." J Am Coll Cardiol 39(7): 1214-9.

Reichelt, A., M. M. Hoeper, et al. (2009). "Chronic thromboembolic pulmonary hypertension: evaluation with 64-detector row CT versus digital substraction angiography." Eur J Radiol 71(1): 49-54.

Rich, S., D. R. Dantzker, et al. (1987). "Primary pulmonary hypertension. A national prospective study." Ann Intern Med 107(2): 216-23.

Rich, S., K. Kieras, et al. (1986). "Antinuclear antibodies in primary pulmonary hypertension." J Am Coll Cardiol 8(6): 1307-11.

Richard, R., E. Lonsdorfer-Wolf, et al. (2001). "Non-invasive cardiac output evaluation during a maximal progressive exercise test, using a new impedance cardiograph device." Eur J Appl Physiol 85(3-4): 202-7.

Rolf F. Kroidl, S. S., Burghart Lehnigk (2009). Kursbuch Spiroergometrie:

Technik und Befundung verständlich gemacht, Georg Thieme Verlag.

Romberg, E. (1891). "Ueber die Sklerose der Lungenarterie." Dsch Archiv Klin Med 48: $197-206$.

Rosenkranz, S. (2007). "Pulmonary hypertension: current diagnosis and treatment." Clin Res Cardiol 96(8): 527-41.

Rosenkranz, S. (2008). "[Novel concepts in the pathobiology of pulmonary arterial hypertension]." Dtsch Med Wochenschr 133 Suppl 6: S167-9.

Ross, R. M. (2003). "ATS/ACCP statement on cardiopulmonary exercise testing." Am J Respir Crit Care Med 167(10): 1451; author reply 1451.

Rubin, L. J. (2002). "Therapy of pulmonary hypertension: the evolution from vasodilators to antiproliferative agents." Am J Respir Crit Care Med 166(10): 1308-9.

Rühle, K. (2008). Praxisleitfaden der Spiroergometrie. Stuttgart, Kohlhammer.

Sitbon, O., M. Humbert, et al. (2005). "Long-term response to calcium channel blockers in idiopathic pulmonary arterial hypertension." Circulation 111(23): 3105-11.

Smith, R. F., G. Johnson, et al. (1993). "Functional capacity in heart failure. Comparison of methods for assessment and their relation to other indexes of heart failure. The V-HeFT VA Cooperative Studies Group." Circulation 87(6 Suppl): VI88-93.

Speich, R. (2011). "Diagnosing pulmonary hypertension: is there a revival of the electrocardiogram?" Eur Respir J 37(5): 994-6.

Sramek, B. B. (1981). Non-invasive technique of measuring cardiac output by means of electrical impedance. 5th International Conference of Electrical Bioimpedance, Tokyo, Japan, 39.

Stojnic, B. B., S. J. Brecker, et al. (1992). "Left ventricular filling characteristics in pulmonary hypertension: a new mode of ventricular interaction." $\underline{\text { Br Heart J }}$ 68(1): 16-20.

Sun, X. G., J. E. Hansen, et al. (2001). "Exercise pathophysiology in patients with primary pulmonary hypertension." Circulation 104(4): 429-35. 
Sutsch, G., W. Kiowski, et al. (1998). "Short-term oral endothelin-receptor antagonist therapy in conventionally treated patients with symptomatic severe chronic heart failure." Circulation 98(21): 2262-8.

Szczeklik, J., A. Szczeklik, et al. (1980). "Haemodynamic changes induced by prostacyclin in man." Br Heart J 44(3): 254-8.

Tei, C., L. H. Ling, et al. (1995). "New index of combined systolic and diastolic myocardial performance: a simple and reproducible measure of cardiac function--a study in normals and dilated cardiomyopathy." J Cardiol 26(6): 357-66.

Teo, K. K., M. D. Hetherington, et al. (1985). "Cardiac output measured by impedance cardiography during maximal exercise tests." Cardiovasc Res 19(12): 737-43.

Tongers, J., B. Schwerdtfeger, et al. (2007). "Incidence and clinical relevance of supraventricular tachyarrhythmias in pulmonary hypertension." Am Heart J 153(1): $127-32$.

Torbicki, A. (2007). "Cardiac magnetic resonance in pulmonary arterial hypertension: a step in the right direction." Eur Heart J 28(10): 1187-9.

Torbicki, A., M. Kurzyna, et al. (2003). "Detectable serum cardiac troponin T as a marker of poor prognosis among patients with chronic precapillary pulmonary hypertension." Circulation 108(7): 844-8.

Trappe, H. J. and H. Lollgen (2000). "[Guidelines for ergometry. German Society of Cardiology--Heart and Cardiovascular Research]." Z Kardiol 89(9): 821-31.

Tunariu, N., S. J. Gibbs, et al. (2007). "Ventilation-perfusion scintigraphy is more sensitive than multidetector CTPA in detecting chronic thromboembolic pulmonary disease as a treatable cause of pulmonary hypertension." $\mathrm{J}$ Nucl Med 48(5): 680-4.

van der Meer, B. J., H. H. Woltjer, et al. (1996). "Impedance cardiography. Importance of the equation and the electrode configuration." Intensive Care Med 22(10): 1120-4.

Veigl, V. L. and W. V. Judy (1983). "Reproducibility of haemodynamic measurements by impedance cardiography." Cardiovasc Res 17(12): 728-34.

Vonk-Noordegraaf, A., J. T. Marcus, et al. (2005). "Interventricular mechanical asynchrony due to right ventricular pressure overload in pulmonary hypertension plays an important role in impaired left ventricular filling." Chest 128(6 Suppl): 628S-630S.

Wasserman, H., Sue et al (1994). Principles of exercise testing and interpretation. Philadelphia, Baltimore, Hongkong, Lea\&Febiger.

Wasserman, K. (1988). "The Dickinson W. Richards lecture. New concepts in assessing cardiovascular function." Circulation 78(4): 1060-71.

Wasserman, K. (2005). Principles of exercise testing and interpretation. Including pathophysiology and clinical applications. Philadelphia, Lippincott Williams \& Wilkins.

Wasserman, K., W. W. Stringer, et al. (1994). "Determination of the anaerobic threshold by gas exchange: biochemical considerations, methodology and physiological effects." Z Kardiol 83 Suppl 3: 1-12.

Westhoff, M. (2007). "[Preoperative functional diagnostics for lung-resecting interventions]." Pneumologie 61(4): 256-63.

Westhoff, M., Rühle, K.-H. (2011). "Ergospirometry." Pneumologe 2011 8: 85-91. 
White, W. B., P. Lund-Johansen, et al. (1990). "Assessment of four ambulatory blood pressure monitors and measurements by clinicians versus intraarterial blood pressure at rest and during exercise." Am J Cardiol 65(1): 60-6.

Wilkens, H. (2010). "Diagnostik der pulmonalen Hypertonie." Pneumologe 2010 7: 174-186.

Wilkens, H., M. Bauer, et al. (2003). "Influence of inhaled iloprost on transpulmonary gradient of big endothelin in patients with pulmonary hypertension." Circulation 107(11): 1509-13.

Wilkens, H., F. Grimminger, et al. "Burden of pulmonary arterial hypertension in Germany." Respir Med 104(6): 902-10.

Winter, U. J., A. K. Gitt, et al. (1994). "[Methodologic aspects of modern, computerized ergospirometry (CPX): ramp program, constant workload test and $\mathrm{CO} 2$ rebreathing method]." Z Kardiol 83 Suppl 3: 13-26.

Wonisch, H., R Pokan, W Kraxner, R Hödl, R Maier, N Watzinger, G Smekal, F M Fruhwald (2003). "Spiroergometry in Cardiology - Physiology and Terminology." J Kardiol 10: 383-90.

Wonisch, M., P. Hofmann, et al. (2002). "Effect of beta(1)-selective adrenergic blockade on maximal blood lactate steady state in healthy men." Eur J Appl Physiol 87(1): 66-71.

Yasunobu, Y., R. J. Oudiz, et al. (2005). "End-tidal PCO2 abnormality and exercise limitation in patients with primary pulmonary hypertension." Chest $\mathbf{1 2 7 ( 5 ) \text { : }}$ 1637-46.

Yeo, T. C., K. S. Dujardin, et al. (1998). "Value of a Doppler-derived index combining systolic and diastolic time intervals in predicting outcome in primary pulmonary hypertension." Am J Cardiol 81(9): 1157-61. 


\section{Anhang}

\section{Blutgasanalyse}

\begin{tabular}{|c|c|c|c|c|}
\hline & Kontrolle & Patienten & PAH & СТЕРН \\
\hline BGA & $\mathrm{MW} \pm \mathrm{SD}$ & $\mathrm{MW} \pm \mathrm{SD}$ & $\mathrm{MW} \pm \mathrm{SD}$ & $\mathrm{MW} \pm \mathrm{SD}$ \\
\hline Ruhe $\mathrm{pO}_{2}$ & $11,21 \pm 1,49$ & $8,78 \pm 1,83^{*}$ & $8,99 \pm 1,80 \#$ & $8,46 \pm 1,93 \#$ \\
\hline Ruhe $\mathrm{pCO}_{2}$ & $4,71 \pm 0,48$ & $4,48 \pm 0,56$ & $4,42 \pm 0,62$ & $4,58 \pm 0,48$ \\
\hline Ruhe pH & $7,43 \pm 0,07$ & $7,46 \pm 0,04(*)$ & $7,47 \pm 0,03 \#$ & $7,46 \pm 0,06$ \\
\hline Ruhe $\mathrm{HCO}_{3}$ & $23,74 \pm 3,30$ & $24,34 \pm 4,06$ & $24,28 \pm 3,89$ & $24,43 \pm 4,53$ \\
\hline Ruhe Laktat & $1,40 \pm 0,43$ & $1,20 \pm 0,14$ & 1,10 (ein Wert) & 1,30 (ein Wert) \\
\hline Belastung $\mathrm{pO}_{2}$ & $10,80 \pm 1,34$ & $7,77 \pm 1,49 *$ & $8,04 \pm 1,50 \#$ & $7,46 \pm 1,48 \#$ \\
\hline Belastung $\mathrm{pCO}_{2}$ & $4,79 \pm 0,49$ & $4,25 \pm 0,74^{*}$ & $4,09 \pm 0,80 \#$ & $4,45 \pm 0,63$ \\
\hline Belastung pH & $7,35 \pm 0,05$ & $7,45 \pm 0,06^{*}$ & $7,45 \pm 0,04 \#$ & $7,45 \pm 0,08 \#$ \\
\hline Belastung $\mathrm{HCO}_{3}$ & $20,16 \pm 2,65$ & $21,94 \pm 3,50^{*}$ & $20,78 \pm 3,10$ & $23,50 \pm 3,55(\#)$ \\
\hline Belastung Laktat & $7,96 \pm 2,37$ & $3,76 \pm 1,75$ & $3,73 \pm 1,47 \#$ & $3,83 \pm 2,56 \#$ \\
\hline
\end{tabular}

Tabelle 34: Blutgasanalyse Kontrolle und Patientengruppen

\section{Lungenfunktion}

\begin{tabular}{|l|c|c|c|c|}
\hline \multirow{2}{*}{ LUFU } & Kontrolle & Patienten & PAH & CTEPH \\
\cline { 2 - 5 } & MW \pm SD & MW \pm SD & MW \pm SD & MW \pm SD \\
\hline FVC & $4,08 \pm 1,27$ & $2,59 \pm 1,02^{*}$ & $2,36 \pm 0,66 \#$ & $2,86 \pm 1,30 \#$ \\
\hline FEV1 & $3,30 \pm 1,18$ & $1,98 \pm 0,89^{*}$ & $1,87 \pm 0,51 \#$ & $2,11 \pm 1,21 \#$ \\
\hline ITGV & $3,27 \pm 0,77$ & $2,90 \pm 0,72$ & $2,70 \pm 0,71$ & $3,19 \pm 0,66$ \\
\hline RV & $2,38 \pm 0,65$ & $2,30 \pm 0,59$ & $2,23 \pm 0,50$ & $2,40 \pm 0,72$ \\
\hline VC & $3,90 \pm 14,0$ & $2,69 \pm 1,04^{*}$ & $2,52 \pm 0,68 \#$ & $2,93 \pm 1,42 \#$ \\
\hline TLC & $6,28 \pm 1,37$ & $5,00 \pm 1,00^{*}$ & $4,75 \pm 0,77 \#$ & $5,36 \pm 1,23$ \\
\hline R eff & $0,24 \pm 0,08$ & $0,37 \pm 0,15^{*}$ & $0,34 \pm 0,12 \#$ & $0,40 \pm 0,19 \#$ \\
\hline SR tot & $1,03 \pm 0,31$ & $1,25 \pm 0,57$ & $1,05 \pm 0,16$ & $1,51 \pm 0,80$ \\
\hline FEV1/VC \% & $73,81 \pm 8,43$ & $72,97 \pm 8,07$ & $74,38 \pm 6,51$ & $70,94 \pm 9,97$ \\
\hline $\begin{array}{l}* \\
*\end{array}<0,05$ PH vs. Kontrolle \\
\# $\mathrm{p}<0,05$ PAH vs. Kontrolle/ CTEPH vs. Kontrolle
\end{tabular}

Tabelle 35: Lungenfunktion Kontrolle und Patientengruppen 


\section{Lebenslauf}

\section{Persönliche Daten:}

Geburtsdatum:

Geburtsort:

Familienstand:

Staatsangehörigkeit:

\section{Studium:}

10/ $2004-11 / 2011$

Studium der Humanmedizin

an der Universität des Saarlandes

Abschluss: Staatsexamen

Berufsausbildung und

Berufspraxis:

seit $01 / 2012$

Assistenzärztin

am Universitätsklinikum des Saarlandes

$08 / 2010-07 / 2011$

Praktisches Jahr im SHG-Klinikum Merzig

$04 / 2005-12 / 2012$

$03 / 2004-10 / 2004$

$01 / 2004-02 / 2004$

$08 / 2001-01 / 2004$

studentische Aushilfskraft im DRK Krankenhaus

Saarlouis

HVB Profil GmbH - Einsatz in der HypoVereinsbank AG in Saarbrücken

Immobilien Service Center der Vereins- und Westbank AG in Hannover

Ausbildung zur Bankkauffrau,

in der Vereins- und Westbank AG (Niederlassung Magdeburg)

Abschluss: Bankkauffrau

\section{Schulbildung:}

$1994-2001$

Janusz-Korczak-Gymnasium Finsterwalde

Abschluss: Hochschulreife 


\section{Publikationen}

\section{Poster:}

1. Nicht-invasive Messung hämodynamischer Parameter bei Patienten mit pulmonal arterieller Hypertonie unter Belastung

K. Hadasch, R. Kaiser, C. Frantz, C. Lensch, R. Bals, H. Wilkens

52. Kongress der deutschen Gesellschaft für Pneumologie und Beatmungsmedizin e.V. (DGP), Dresden, 07.04.-10.04.2011

2. Nicht-invasive Messung hämodynamischer Parameter bei Patienten mit pulmonal arterieller Hypertonie unter Belastung

K. Hadasch, R. Kaiser, C. Frantz, C. Lensch, R. Bals, H. Wilkens

117. Kongress der Deutsche Gesellschaft für Innere Medizin i.V. (DGIM), Wiesbaden, 30.04.-03.05.2012

\section{Vortrag:}

Nicht-invasive Messung hämodynamischer Parameter bei Patienten mit pulmonaler Hypertonie unter Belastung

K. Hadasch, R. Kaiser, C. Frantz, C. Lensch, R. Bals, H. Wilkens

52. Kongress der deutschen Gesellschaft für Pneumologie und Beatmungsmedizin e.V. (DGP), Dresden, 07.04.-10.04.2011 


\section{Danksagung}

Mein Dank geht an Herrn Prof. Dr. med. Dr. rer. nat. Robert Bals, Direktor der Klinik für Innere Medizin V am Universitätsklinikum des Saarlandes in Homburg dafür, dass es mir ermöglicht wurde diese Dissertation dort zu erstellen.

Ebenfalls bedanke ich mich bei Frau Prof. Dr. Heinrike Wilkens, für die Überlassung des Themas und die Unterstützung bei der Anfertigung dieser Arbeit.

Mein ganz besonderer Dank gilt Dr. Ralf Kaiser für die Betreuung und Unterstützung während der Datenerhebung und Erstellung der Arbeit. Er hat mir bei auftretenden Schwierigkeiten und Fragen sehr hilfreich zur Seite gestanden und das Voranschreiten der Arbeit mit persönlichem Engagement gefördert.

Die spiroergometrischen Untersuchungen und Bioimpedanzmessungen erfolgten in den Untersuchungsräumen der Inneren Medizin V der Universitätskliniken des Saarlandes. Ich danke vor allem den Mitarbeiterinnen Frau Anneliese Ferner und Frau Martina Dollwett, die an allen Messungen tatkräftig mitwirkten.

Weiterer Dank gilt Stephanie Matthiesen und Frederik Reinke für das Korrekturlesen dieser Arbeit.

Auch geht mein besonderer Dank an meine Familie, meine Freunde und meinen Partner Markus Mayer, der mich stets bestärkt hat, wenn ich an mir gezweifelt habe und mich nicht nur tatkräftig unterstützte, sondern auch stets aufbaute und für die erforderliche Abwechslung sorgte. 